\title{
HOWE DUALITY AND KOSTANT HOMOLOGY FORMULA FOR INFINITE-DIMENSIONAL LIE SUPERALGEBRAS
}

\author{
SHUN-JEN CHENG ${ }^{\dagger}$ AND JAE-HOON KWON ${ }^{\dagger \dagger}$
}

\begin{abstract}
Using Howe duality we compute explicitly Kostant-type homology groups for a wide class of representations of the infinite-dimensional Lie superalgebra $\widehat{\mathfrak{g l}}_{\infty \mid \infty}$ and its classical subalgebras at positive integral levels. We also obtain Kostant-type homology formulas for the Lie algebra $\widehat{\mathfrak{g l}}_{\infty}$ at negative integral levels. We further construct resolutions in terms of generalized Verma modules for these representations.
\end{abstract}

\section{Contents}

1. Introduction

2. Infinite-dimensional Lie algebras

2.1. Notation and conventions

2.2. Preliminaries on $\widehat{\mathfrak{g l}}_{\infty}$ and its subalgebras of classical types

2.3. Classical dual pairs on infinite-dimensional Fock spaces

2.4. $\mathfrak{u}_{-}$-homology groups of $\mathfrak{g}$-modules

3. Infinite-dimensional Lie superalgebras

3.1. The Lie superalgebra $\widehat{\mathfrak{g l}}_{\infty \mid \infty}$ and its subalgebras of classical types

3.2. Tensor representations of $\mathfrak{g l}(m \mid n)$ and hook Schur functions

3.3. Super dual pairs on infinite-dimensional Fock spaces

4. The Casimir operators

4.1. The bilinear form $(\cdot \mid \cdot)_{c}$ and the Casimir operator $\Omega$ of $\mathfrak{g}$

4.2. The bilinear form $(\cdot \mid \cdot)_{s}$ and the Casimir operator $\bar{\Omega}$ of $\overline{\mathfrak{g}}$

4.3. $\overline{\mathfrak{u}}_{-}$-homology groups of $\overline{\mathfrak{g}}$-modules

5. Computation of $\overline{\mathfrak{u}}_{-}$-homology groups

5.1. Comparison of Casimir eigenvalues

5.2. Formulas for the $\overline{\mathfrak{u}}_{-}$-homology groups

6. Resolution in terms of generalized Verma modules

7. $\mathfrak{u}_{-}$-homology formula of $\widehat{\mathfrak{g l}}_{\infty}$-modules at negative integral levels

8. Index of notation in alphabetical order

\footnotetext{
${ }^{\dagger}$ Partially supported by an NSC-grant and an Academia Sinica Investigator grant.

${ }^{\dagger \dagger}$ Partially supported by KRF-grant 2005-070-C00004.
} 


\section{INTRODUCTION}

Howe duality [H1, H2] has found important applications in many areas of mathematics where representation theory is an indispensable tool. It is therefore not surprising that a concept as simple and fundamental should prove to be useful and powerful in the representation theory of Lie superalgebras. The first Howe dualities involving Lie superalgebras already appeared in Howe's original paper [H1]. It was followed by a systematic and in-depth study of such dualities in [CW1, CW2, LS, S2]. These works mainly were concerned with the construction of Howe dualities involving Lie superalgebras. Subsequent works focused on their applications; in particular, we mention here the derivation of combinatorial character formulas for Lie superalgebras in [CL, CZ2].

The purpose of the present paper is to demonstrate yet another application of Howe duality to Lie superalgebras, namely the computation of certain Kostant homology groups (and thus cohomology groups) [Ko]. As examples we compute them for a large class of irreducible representations of $\widehat{\mathfrak{g l}}_{\infty \mid \infty}(\mathrm{cf}$. [KvL,,$\overline{\mathrm{KW}}$ ) ) and its subalgebras of classical types $\mathfrak{b}, \mathfrak{c}, \mathfrak{d}$. Similar to character formulas, it is a rather non-trivial task to compute such homology and cohomology groups for Lie (super)algebras directly. Indeed such formulas and the Euler-Poincaré principle immediately imply character formulas as well.

Let us provide more details below. In the remainder of this section it will be convenient to restrict most of our discussion to the case of $\widehat{\mathfrak{g l}}_{\infty \mid \infty}$ for simplicity's sake. Consider the Lie algebra $\mathfrak{g l}_{\infty}$ consisting of matrices $\left(a_{i j}\right)$ with $i, j \in \mathbb{Z}$, and $a_{i j}=0$ for all but finitely many $a_{i j}$ 's. Let $\mathfrak{g}=\widehat{\mathfrak{g l}}_{\infty}$ denote its well-known central extension by a one-dimensional center (see (2.3)). Topological completions of $\mathfrak{g}$, and its subalgebras of classical types $\mathfrak{b}, \mathfrak{c}, \mathfrak{d}$, have found remarkable applications in mathematical physics since their introduction by the Kyoto school [DJKM], and the results in this paper remain valid for these completions as well, since all irreducible representations considered in this paper are also irreducible representations of the corresponding completions. Introduce the subalgebras $\mathfrak{g l}_{\leq 0}, \mathfrak{g l}_{>0}$ and $\mathfrak{u}_{-}$consisting of matrices $\left(a_{i j}\right)$ with $i, j \leq 0, i, j>0$ and $i>0, j \leq 0$, respectively. Let $\mathfrak{F}^{\ell}$ denote the Fock space generated by $\ell$ pairs of free fermions. Then it is known that there is a joint action of $\mathfrak{g} \times \mathrm{GL}(\ell)$ on $\mathfrak{F}^{\ell}$. Indeed $(\mathfrak{g}, \mathrm{GL}(\ell))$ forms a reductive dual pair $[\mathrm{F}$, giving a one-to-one correspondence between certain irreducible highest weight representations of $\mathfrak{g}$ and the irreducible rational representations of $\operatorname{GL}(\ell)$. Now the irreducible rational representations of $\mathrm{GL}(\ell)$ are parameterized by $\mathcal{P}(\mathrm{GL}(\ell))$, the 
set of generalized partitions of length $\ell$. We denote the $\mathfrak{g}$-module corresponding to $\lambda \in \mathcal{P}(\mathrm{GL}(\ell))$ by $L\left(\mathfrak{g}, \Lambda^{\mathfrak{a}}(\lambda)\right)$. Since the derived subalgebra is a generalized Kac-Moody algebra, the homology theory of integrable modules over generalized Kac-Moody algebra (cf. [J]) is applicable to $\mathfrak{g}$, giving us a precise description of the $\mathfrak{g l}_{\leq 0} \oplus \mathfrak{g l}_{>0}$-module structure of the $\mathfrak{u}_{-}$-homology groups $\mathrm{H}_{k}\left(\mathfrak{u}_{-} ; L\left(\mathfrak{g}, \Lambda^{\mathfrak{a}}(\lambda)\right)\right)$ for $k \in \mathbb{N}$.

Now consider the super analogue $\mathfrak{g l}_{\infty \mid \infty}$ consisting of matrices $\left(a_{r s}\right)$ with $r, s \in \frac{1}{2} \mathbb{Z}$, and $a_{r s}=0$ for all but finitely many $a_{r s}$ 's. We regard $\left(a_{r s}\right)$ as the matrix of a linear transformation on the space $\mathbb{C}^{\infty} \mid \infty$ with respect to a basis $\left\{e_{r} \mid r \in \frac{1}{2} \mathbb{Z}\right\}$, where the $\mathbb{Z}_{2}$-gradation is given by $\operatorname{deg} e_{r}=2 r$ modulo 2 . There is a similar central extension $\overline{\mathfrak{g}}=\widehat{\mathfrak{g l}}_{\infty \mid \infty}\left(\right.$ see $(3.1)$ ), and we can define subalgebras $\overline{\mathfrak{g l}}_{\leq 0}, \overline{\mathfrak{g l}}_{>0}$, and $\overline{\mathfrak{u}}_{-}$ in an analogous fashion. Let $\overline{\mathfrak{F}}$ denote the Fock space generated by $\ell$ pairs of free fermions and $\ell$ pairs of free bosons. Then there exists a joint action of $\overline{\mathfrak{g}} \times \operatorname{GL}(\ell)$ on $\overline{\mathfrak{F}}^{\ell}$, which also forms a Howe dual pair [CW2. This provides another one-toone correspondence between certain irreducible highest weight representations of $\overline{\mathfrak{g}}$ and the irreducible rational representations of $\mathrm{GL}(\ell)$. We denote the $\overline{\mathfrak{g}}$-module corresponding to $\lambda \in \mathcal{P}(\operatorname{GL}(\ell))$ by $L\left(\overline{\mathfrak{g}}, \bar{\Lambda}^{\mathfrak{a}}(\lambda)\right)$. Thus these two Howe dualities establish a one-to-one correspondence

$$
L\left(\mathfrak{g}, \Lambda^{\mathfrak{a}}(\lambda)\right) \stackrel{1-1}{\longleftrightarrow} L\left(\overline{\mathfrak{g}}, \bar{\Lambda}^{\mathfrak{a}}(\lambda)\right), \quad \lambda \in \mathcal{P}(\mathrm{GL}(\ell)) .
$$

This correspondence enables us to determine the $\overline{\mathfrak{g l}}_{\leq 0} \oplus \overline{\mathfrak{g l}}_{>0}$-module structure of $\mathrm{H}_{k}\left(\overline{\mathfrak{u}}_{-} ; L\left(\overline{\mathfrak{g}}, \bar{\Lambda}^{\mathfrak{a}}(\lambda)\right)\right)$ in terms $\mathrm{H}_{k}\left(\mathfrak{u}_{-} ; L\left(\mathfrak{g}, \Lambda^{\mathfrak{a}}(\lambda)\right)\right)$, for all $k \in \mathbb{N}$, in Theorem 5.1 . We note that in contrast to Lie algebras the derived subalgebra of $\widehat{\mathfrak{g l}}_{\infty \mid \infty}$ is not a generalized Kac-Moody superalgebra, and thus a direct generalization of the classical homology theory is not possible.

To our best knowledge such a similar relationship on the level of homology groups between Lie algebras and Lie superalgebras was first observed for the irreducible tensor representations of the general linear superalgebra in [CZ1]. It is our hope that the results presented here may lead to a better understanding of the relationship between the representation theories of Lie algebras and Lie superalgebras (e.g. in the spirit of [CWZ]), which is our motivation for this investigation.

Besides Howe duality involving an infinite-rank affine Kac-Moody algebras, worked out systematically in [W], Aribaud's method for computing homology from the character formula [A, L] also plays an important role in our approach. Together with elementary symmetric function theory they provide us with all the necessary tools to reduce the problem to a comparison of eigenvalues of the classical and the super Casimir operators on the chain spaces. 
We conclude with an outline of the paper. In Sections 2 and 3 the Howe dualities involving the infinite-dimensional Lie (super)algebras are recalled. In Section 3.2 we discuss in detail the category of tensor representations of $\mathfrak{g l}(m \mid n)$, for $n \in \mathbb{N} \cup\{\infty\}$. Although the results therein may be known to experts, we have decided to include them, as we were unable to find references for them. These results are then used in subsequent sections. In Section 4 invariant symmetric non-degenerate bilinear forms are introduced for the Lie algebras and superalgebras under discussion, based on which the Casimir operators are defined. We remark that our Casimir operators differ slightly from the usual ones, as this makes subsequent comparisons of their eigenvalues simpler. The homology groups for $\widehat{\mathfrak{g l}}_{\infty \mid \infty}$ and its classical subalgebras are then computed in Section 5. In Section 6 resolutions in terms of generalized Verma modules are constructed. In Section 7 the $\widehat{\mathfrak{g l}}_{\infty}$-modules appearing in the bosonic Fock space are discussed, and their Kostant-type homology groups are computed. In Section 8 we collect some notation for the convenience of the reader.

Finally, all vector spaces, algebras et cetera are over the complex numbers $\mathbb{C}$.

Acknowledgements. The first author thanks NCTS for partial support. The second author thanks the Institute of Mathematics, Academia Sinica, for hospitality and support, where part of this work was completed. Both authors thank the referees for helpful comments.

\section{Infinite-Dimensional LiE ALGEBRAS}

2.1. Notation and conventions. Denote by $\mathcal{P}^{+}$the set of partitions. Given $\ell \in \mathbb{N}$, a non-increasing sequence $\lambda=\left(\lambda_{1}, \lambda_{2}, \cdots, \lambda_{\ell}\right)$ of $\ell$ integers with

$$
\lambda_{1} \geq \lambda_{2} \geq \cdots \geq \lambda_{i}>0=\cdots=0>\lambda_{j} \geq \cdots \geq \lambda_{\ell}
$$

will be called a generalized partition of length $\ell$. Similar to ordinary partitions, a generalized partition $\lambda$ may be viewed as a left-adjusted diagram with $\ell$ rows such that the $k$-th row contains $\lambda_{k}$ cells. Here we use the convention that $\lambda_{k} \geq 0$ (resp. $\left.\lambda_{k} \leq 0\right)$ means we have $\left|\lambda_{k}\right|$ boxes to the right (resp. left) of a fixed vertical line indicating 0 cells. As an example consider $\lambda=(5,3,2,1,-1,-2)$ with $\ell=6$. The corresponding diagram is given in (2.2) below.

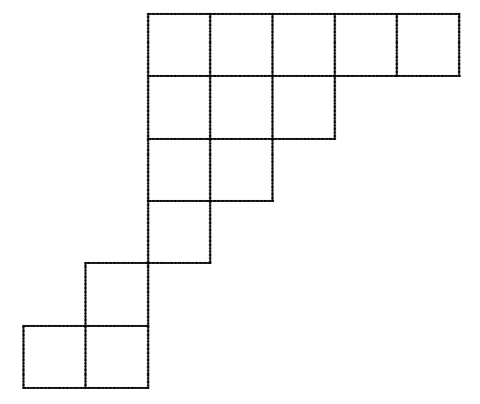


For a generalized partition $\lambda$ of length $\ell$, let $\lambda_{k}^{\prime}$ be the length of the $k$-th column of $\lambda$. We use the convention that the first column of $\lambda$ of the form (2.1) is the first column of the partition $\lambda_{1} \geq \lambda_{2} \geq \cdots \geq \lambda_{j-1}$. The column to its right is the second column of $\lambda$, while the column to its left is the zeroth column and the column to the left of its zeroth column is the -1 -st column et cetera. We also use the convention that a non-positive column has non-positive length. For our example (2.2), we have $\lambda_{-1}^{\prime}=-1, \lambda_{0}^{\prime}=-2, \lambda_{1}^{\prime}=4$ et cetera. The size of a generalized partition $\lambda$ is $\sum_{j} \lambda_{j}$, and is denoted by $|\lambda|$. For a partition $\lambda=\left(\lambda_{1}, \lambda_{2}, \ldots\right), \lambda^{\prime}=\left(\lambda_{1}^{\prime}, \lambda_{2}^{\prime}, \ldots\right)$ and $l(\lambda)$ denote the conjugate and the length of $\lambda$, respectively.

2.2. Preliminaries on $\widehat{\mathfrak{g l}}_{\infty}$ and its subalgebras of classical types. Below we recall the infinite-dimensional Lie algebra $\widehat{\mathfrak{g l}}_{\infty}$ and its subalgebras $\mathfrak{b}_{\infty}, \mathfrak{c}_{\infty}, \mathfrak{d}_{\infty}$. We will say that these algebras are of types $\mathfrak{a}, \mathfrak{b}, \mathfrak{c}, \mathfrak{d}$, respectively. The following notation will be assumed throughout the paper.

$\cdot \mathfrak{g}$ : the Lie algebra $\widehat{\mathfrak{g l}}_{\infty}$, or its subalgebra $\mathfrak{b}_{\infty}, \mathfrak{c}_{\infty}, \mathfrak{d}_{\infty}$,

$\cdot \mathfrak{h}$ : a Cartan subalgebra of $\mathfrak{g}$,

- $I$ : the index set for simple roots,

- $\Pi=\left\{\alpha_{i} \mid i \in I\right\}$ : the set of simple roots,

$\cdot \Pi^{\vee}=\left\{\alpha_{i}^{\vee} \mid i \in I\right\}$ : the set of simple coroots,

$\cdot \Delta^{+}$: the set of positive roots.

2.2.1. The Lie algebra $\widehat{\mathfrak{g l}}_{\infty}$. Let $\mathbb{C}^{\infty}$ be the vector space over $\mathbb{C}$ with a basis $\left\{e_{i} \mid i \in\right.$ $\mathbb{Z}\}$ so that an element in $\operatorname{End}\left(\mathbb{C}^{\infty}\right)$ may be identified with a matrix $\left(a_{i j}\right)(i, j \in \mathbb{Z})$. Let $E_{i j}$ be the matrix with 1 at the $i$-th row and $j$-th column and zero elsewhere. Let $\mathfrak{g l}_{\infty}$ denote the subalgebra consisting of $\left(a_{i j}\right)$ with finitely many non-zero $a_{i j}$ 's. Then $\mathfrak{g l}_{\infty}$ in matrix form is spanned by $E_{i j}(i, j \in \mathbb{Z})$. Denote by $\widehat{\mathfrak{g l}}_{\infty}=\mathfrak{g l}_{\infty} \oplus \mathbb{C} K$ the central extension of $\mathfrak{g l}_{\infty}$ by a one-dimensional center $\mathbb{C} K$ given by the 2 -cocycle (cf. DJKM, K2])

$$
\alpha(A, B):=\operatorname{Tr}([J, A] B)
$$

where $J=\sum_{i \leq 0} E_{i i}$. The derived subalgebra of $\widehat{\mathfrak{g l}}_{\infty}$ is an infinite-rank Kac-Moody algebra [K2]. Note that $\mathfrak{h}=\sum_{i \in \mathbb{Z}} \mathbb{C} E_{i i} \oplus \mathbb{C} K$. Put $I=\mathbb{Z}$. Then we have

$$
\begin{aligned}
\Pi^{\vee} & =\left\{\alpha_{i}^{\vee}=E_{i i}-E_{i+1, i+1}+\delta_{i 0} K(i \in I)\right\}, \\
\Pi & =\left\{\alpha_{i}=\epsilon_{i}-\epsilon_{i+1}(i \in I)\right\}, \\
\Delta^{+} & =\left\{\epsilon_{i}-\epsilon_{j}(i, j \in I, i<j)\right\},
\end{aligned}
$$


where $\epsilon_{i} \in \mathfrak{h}^{*}$ is determined by $\left\langle\epsilon_{i}, E_{j j}\right\rangle=\delta_{i j}$ and $\left\langle\epsilon_{i}, K\right\rangle=0$. Furthermore, for $i \in I$, let $\Lambda_{i}^{\mathfrak{a}}$ be the $i$-th fundamental weight of $\widehat{\mathfrak{g l}}_{\infty}$. That is, we have

$$
\Lambda_{i}^{\mathfrak{a}}= \begin{cases}\Lambda_{0}^{\mathfrak{a}}-\sum_{k=i+1}^{0} \epsilon_{k}, & \text { if } i<0, \\ \Lambda_{0}^{\mathfrak{a}}+\sum_{k=1}^{i} \epsilon_{k}, & \text { if } i>0,\end{cases}
$$

where $\Lambda_{0}^{\mathfrak{a}} \in \mathfrak{h}^{*}$ is determined by $\left\langle\Lambda_{0}^{\mathfrak{a}}, K\right\rangle=1$ and $\left\langle\Lambda_{0}^{\mathfrak{a}}, E_{j j}\right\rangle=0$, for all $j \in \mathbb{Z}$. Let $\rho_{c} \in \mathfrak{h}^{*}$ be determined by $\left\langle\rho_{c}, E_{j j}\right\rangle=-j$, for all $j \in \mathbb{Z}$, and $\left\langle\rho_{c}, K\right\rangle=0$, so that we have $\left\langle\rho_{c}, \alpha_{i}^{\vee}\right\rangle=1$, for all $i \in I$.

By assigning degree 0 to the Cartan subalgebra and setting $\operatorname{deg} E_{i j}=j-i$, we equip $\widehat{\mathfrak{g l}}_{\infty}$ with a $\mathbb{Z}$-gradation: $\widehat{\mathfrak{g l}}_{\infty}=\bigoplus_{k \in \mathbb{Z}}\left(\widehat{\mathfrak{g l}}_{\infty}\right)_{k}$. This leads to the following triangular decomposition:

$$
\widehat{\mathfrak{g l}}_{\infty}=\left(\widehat{\mathfrak{g l}}_{\infty}\right)_{+} \oplus\left(\widehat{\mathfrak{g l}}_{\infty}\right)_{0} \oplus\left(\widehat{\mathfrak{g l}}_{\infty}\right)_{-}
$$

where $\left(\widehat{\mathfrak{g l}}_{\infty}\right)_{ \pm}=\bigoplus_{k \in \pm \mathbb{N}}\left(\widehat{\mathfrak{g l}}_{\infty}\right)_{k}$ and $\left(\widehat{\mathfrak{g l}}_{\infty}\right)_{0}=\mathfrak{h}$.

2.2.2. The Lie algebras $\mathfrak{b}_{\infty}, \mathfrak{c}_{\infty}, \mathfrak{d}_{\infty}$. For convenience of the reader we will briefly review the classical subalgebras of $\widehat{\mathfrak{g l}}_{\infty}$ (cf. [K2]). For $\mathfrak{x} \in\{\mathfrak{b}, \mathfrak{c}, \mathfrak{d}\}$, let $\overline{\mathfrak{x}}_{\infty}$ be the subalgebra of $\mathfrak{g l}_{\infty}$ preserving the following bilinear form on $\mathbb{C}^{\infty}$ :

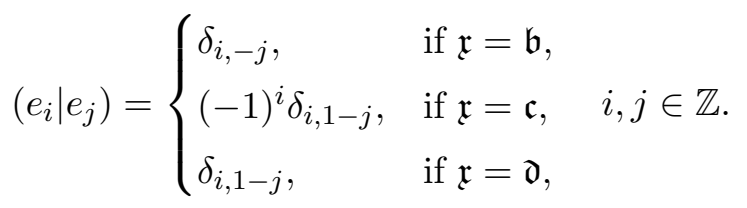

Let $\mathfrak{x}_{\infty}=\overline{\mathfrak{x}}_{\infty} \oplus \mathbb{C} K$ be the central extension of $\overline{\mathfrak{x}}_{\infty}$ determined by the restriction of the two-cocycle (2.3). Then $\mathfrak{x}_{\infty}$ has a natural triangular decomposition induced from $\widehat{\mathfrak{g l}}_{\infty}$ :

$$
\mathfrak{x}_{\infty}=\left(\mathfrak{x}_{\infty}\right)_{+} \oplus\left(\mathfrak{x}_{\infty}\right)_{0} \oplus\left(\mathfrak{x}_{\infty}\right)_{-},
$$

where $\left(\mathfrak{x}_{\infty}\right)_{ \pm}=\mathfrak{x}_{\infty} \cap\left(\widehat{\mathfrak{g l}}_{\infty}\right)_{ \pm}$and $\left(\mathfrak{x}_{\infty}\right)_{0}=\mathfrak{x}_{\infty} \cap\left(\widehat{\mathfrak{g l}}_{\infty}\right)_{0}$. For $i \in \mathbb{N}$, let

$$
\widetilde{E}_{i}= \begin{cases}E_{i i}-E_{-i,-i}, & \text { if } \mathfrak{x}=\mathfrak{b}, \\ E_{i i}-E_{1-i, 1-i}, & \text { if } \mathfrak{x}=\mathfrak{c}, \mathfrak{d} .\end{cases}
$$

Note that $\mathfrak{h}=\left(\mathfrak{x}_{\infty}\right)_{0}=\sum_{i \in \mathbb{N}} \mathbb{C} \widetilde{E}_{i} \oplus \mathbb{C} K$. We may regard $\epsilon_{i} \in\left(\widehat{\mathfrak{g l}}_{\infty}\right)_{0}^{*}$ as an element in $\left(\mathfrak{x}_{\infty}\right)_{0}^{*}$ via restriction so that $\left\langle\epsilon_{i}, \widetilde{E}_{j}\right\rangle=\delta_{i j}$ for $i, j \in \mathbb{N}$. Put $I=\mathbb{Z}_{+}$. Then we have

- $\mathfrak{b}_{\infty}$

$$
\begin{aligned}
\Pi^{\vee} & =\left\{\alpha_{0}^{\vee}=-2 \widetilde{E}_{1}+2 K, \alpha_{i}^{\vee}=\widetilde{E}_{i}-\widetilde{E}_{i+1}(i \in \mathbb{N})\right\}, \\
\Pi & =\left\{\alpha_{0}=-\epsilon_{1}, \alpha_{i}=\epsilon_{i}-\epsilon_{i+1}(i \in \mathbb{N})\right\}, \\
\Delta^{+} & =\left\{ \pm \epsilon_{i}-\epsilon_{j}, \quad-\epsilon_{i}(i, j \in \mathbb{N}, i<j)\right\} .
\end{aligned}
$$


- $\mathfrak{c}_{\infty}$

$$
\begin{aligned}
\Pi^{\vee} & =\left\{\alpha_{0}^{\vee}=-\widetilde{E}_{1}+K, \alpha_{i}^{\vee}=\widetilde{E}_{i}-\widetilde{E}_{i+1}(i \in \mathbb{N})\right\}, \\
\Pi & =\left\{\alpha_{0}=-2 \epsilon_{1}, \alpha_{i}=\epsilon_{i}-\epsilon_{i+1}(i \in \mathbb{N})\right\}, \\
\Delta^{+} & =\left\{ \pm \epsilon_{i}-\epsilon_{j},-2 \epsilon_{i}(i, j \in \mathbb{N}, i<j)\right\} .
\end{aligned}
$$

$\bullet \mathfrak{d}_{\infty}$

$$
\begin{aligned}
\Pi^{\vee} & =\left\{\alpha_{0}^{\vee}=-\widetilde{E}_{1}-\widetilde{E}_{2}+2 K, \alpha_{i}^{\vee}=\widetilde{E}_{i}-\widetilde{E}_{i+1}(i \in \mathbb{N})\right\}, \\
\Pi & =\left\{\alpha_{0}=-\epsilon_{1}-\epsilon_{2}, \alpha_{i}=\epsilon_{i}-\epsilon_{i+1}(i \in \mathbb{N})\right\}, \\
\Delta^{+} & =\left\{ \pm \epsilon_{i}-\epsilon_{j}(i, j \in \mathbb{N}, i<j)\right\} .
\end{aligned}
$$

For $i \in I$, we denote by $\Lambda_{i}^{\mathfrak{x}}$ the $i$-th fundamental weight for $\mathfrak{x}_{\infty}$, that is, $\left\langle\Lambda_{i}^{\mathfrak{x}}, \alpha_{j}^{\vee}\right\rangle=$ $\delta_{i j}(j \in I)$, where $\Lambda_{0}^{\mathfrak{x}} \in \mathfrak{h}^{*}$ is determined by $\left\langle\Lambda_{0}^{\mathfrak{x}}, \widetilde{E}_{i}\right\rangle=0$ for $i \in \mathbb{N}$ and $\left\langle\Lambda_{0}^{\mathfrak{x}}, K\right\rangle=r$ with $r=\frac{1}{2}, 1, \frac{1}{2}$, for $\mathfrak{x}=\mathfrak{b}, \mathfrak{c}, \mathfrak{d}$, respectively. In fact, we have

$$
\begin{aligned}
& \Lambda_{i}^{\mathfrak{b}}=2 \Lambda_{0}^{\mathfrak{b}}+\epsilon_{1}+\cdots+\epsilon_{i}, \quad i \geq 1, \\
& \Lambda_{i}^{\mathfrak{c}}=\Lambda_{0}^{\mathfrak{c}}+\epsilon_{1}+\cdots+\epsilon_{i}, \quad i \geq 1, \\
& \Lambda_{i}^{\mathfrak{d}}= \begin{cases}\Lambda_{0}^{\mathfrak{d}}+\epsilon_{1}, & \text { if } i=1, \\
2 \Lambda_{0}^{\mathfrak{d}}+\epsilon_{1}+\cdots+\epsilon_{i}, & \text { if } i>1\end{cases}
\end{aligned}
$$

We let $\rho_{c} \in \mathfrak{h}^{*}$ be determined by

$$
\left\langle\rho_{c}, \widetilde{E}_{j}\right\rangle=\left\{\begin{array}{ll}
-j+\frac{1}{2}, & \text { for } \mathfrak{b}_{\infty}, \\
-j, & \text { for } \mathfrak{c}_{\infty}, \mathfrak{d}_{\infty},
\end{array} \quad j \in \mathbb{N}, \quad\left\langle\rho_{c}, K\right\rangle=\left\{\begin{array}{cl}
0, & \text { for } \mathfrak{b}_{\infty}, \mathfrak{c}_{\infty}, \\
-1, & \text { for } \mathfrak{d}_{\infty}
\end{array}\right.\right.
$$

2.3. Classical dual pairs on infinite-dimensional Fock spaces. Let $\mathfrak{g}$ be one of the Lie algebras given in Section 2.2. Let $\Lambda \in \mathfrak{h}^{*}$ be given. By standard arguments there is a unique irreducible highest weight representation of $\mathfrak{g}$ of highest weight $\Lambda$, which will be denoted by $L(\mathfrak{g}, \Lambda)$.

We fix a positive integer $\ell \geq 1$ and consider $\ell$ pairs of free fermions $\psi^{ \pm, i}(z)$ with $i=1, \cdots, \ell$. That is, we have

$$
\psi^{+, i}(z)=\sum_{n \in \mathbb{Z}} \psi_{n}^{+, i} z^{-n-1}, \quad \psi^{-, i}(z)=\sum_{n \in \mathbb{Z}} \psi_{n}^{-, i} z^{-n},
$$

with non-trivial commutation relations $\left[\psi_{m}^{+, i}, \psi_{n}^{-, j}\right]=\delta_{i j} \delta_{m+n, 0}$. Let $\mathfrak{F}^{\ell}$ denote the corresponding Fock space generated by the vaccum vector $|0\rangle$, which is annihilated by $\psi_{n}^{+, i}, \psi_{m}^{-, i}$ for $n \geq 0$ and $m>0$.

We introduce a neutral fermionic field $\phi(z)=\sum_{n \in \mathbb{Z}} \phi_{n} z^{-n-1}$ with non-trivial commutation relations $\left[\phi_{m}, \phi_{n}\right]=\delta_{m+n, 0}$. Denote by $\mathfrak{F}^{\frac{1}{2}}$ the Fock space of $\phi(z)$ 
generated by a vacuum vector that is annihilated by $\phi_{m}$ for $m \geq 0$. We denote by $\mathfrak{F}^{\ell+\frac{1}{2}}$ the tensor product of $\mathfrak{F}^{\ell}$ and $\mathfrak{F}^{\frac{1}{2}}$.

We assume that $x_{n}(n \in \mathbb{Z})$ and $z_{i}(i=1, \ldots, \ell)$ are formal indeterminates.

2.3.1. The $\left(\widehat{\mathfrak{g l}}_{\infty}, \mathrm{GL}(\ell)\right)$-duality. Let $\mathrm{GL}(\ell)$ be the general linear group of rank $\ell$. The space of complex $\ell \times \ell$ matrices forms the Lie algebra $\mathfrak{g l}(\ell)$ of $\operatorname{GL}(\ell)$. We denote by $e_{i j}(1 \leq i, j \leq \ell)$ the elementary matrix with 1 in the $i$-th row and $j$-th column and 0 elsewhere. Then $H=\sum_{i} \mathbb{C} e_{i i}$ is a Cartan subalgebra, while $\sum_{i \leq j} \mathbb{C} e_{i j}$ is a Borel subalgebra containing $H$. Recall that an irreducible rational representations of $\mathfrak{g l}(\ell)$ (or $\mathrm{GL}(\ell)$ ) is determined by its highest weight $\lambda \in H^{*}$ with $\left\langle\lambda, e_{i i}\right\rangle=\lambda_{i} \in \mathbb{Z}(1 \leq i \leq \ell)$ and $\lambda_{1} \geq \ldots \geq \lambda_{\ell}$. Denote by $V_{\mathrm{GL}(\ell)}^{\lambda}$ the irreducible representation corresponding to $\lambda$. Identifying $\lambda$ with $\left(\lambda_{1}, \ldots, \lambda_{\ell}\right)$ the irreducible rational representations of $\mathrm{GL}(\ell)$ are parameterized by

$$
\mathcal{P}(\mathrm{GL}(\ell)):=\left\{\lambda=\left(\lambda_{1}, \cdots, \lambda_{\ell}\right) \mid \lambda_{i} \in \mathbb{Z}, \lambda_{1} \geq \ldots \geq \lambda_{\ell}\right\},
$$

which is precisely the set of generalized partitions of length $\ell$.

Proposition 2.1. [F] (cf. [W], Theorem 3.1]) There exists an action of $\widehat{\mathfrak{g l}}_{\infty} \times \mathrm{GL}(\ell)$ on $\mathfrak{F}^{\ell}$. Furthermore, under this joint action, we have

$$
\mathfrak{F}^{\ell} \cong \bigoplus_{\lambda \in \mathcal{P}(\mathrm{GL}(\ell))} L\left(\widehat{\mathfrak{g l}}_{\infty}, \Lambda^{\mathfrak{a}}(\lambda)\right) \otimes V_{\mathrm{GL}(\ell)}^{\lambda}
$$

where $\Lambda^{\mathfrak{a}}(\lambda)=\ell \Lambda_{0}^{\mathfrak{a}}+\sum_{j \in \mathbb{Z}} \lambda_{j}^{\prime} \epsilon_{j}=\sum_{i=1}^{\ell} \Lambda_{\lambda_{i}}^{\mathfrak{a}}$.

Computing the trace of the operator $\prod_{n \in \mathbb{Z}} x_{n}^{E_{n n}} \prod_{i=1}^{\ell} z_{i}^{e_{i i}}$ on both sides of (2.4), we obtain the following identity:

$$
\prod_{i=1}^{\ell} \prod_{n \in \mathbb{N}}\left(1+x_{n} z_{i}\right)\left(1+x_{1-n}^{-1} z_{i}^{-1}\right)=\sum_{\lambda \in \mathcal{P}(\mathrm{GL}(\ell))} \operatorname{ch} L\left(\widehat{\mathfrak{g l}}_{\infty}, \Lambda^{\mathfrak{a}}(\lambda)\right) \operatorname{ch} V_{\mathrm{GL}(\ell)}^{\lambda} .
$$

2.3.2. The $\left(\mathfrak{c}_{\infty}, \operatorname{Sp}(2 \ell)\right)$-duality. Let $\operatorname{Sp}(2 \ell)$ denote the symplectic group, which is the subgroup of $\mathrm{GL}(2 \ell)$ preserving the non-degenerate skew-symmetric bilinear form on $\mathbb{C}^{2 \ell}$ given by

$$
\left(\begin{array}{cc}
0 & J_{\ell} \\
-J_{\ell} & 0
\end{array}\right)
$$

where $J_{\ell}$ is the following $\ell \times \ell$ matrix:

$$
J_{\ell}=\left(\begin{array}{ccccc}
0 & 0 & \cdots & 0 & 1 \\
0 & 0 & \cdots & 1 & 0 \\
\vdots & \vdots & \vdots & \vdots & \vdots \\
0 & 1 & \cdots & 0 & 0 \\
1 & 0 & \cdots & 0 & 0
\end{array}\right)
$$


Let $\mathfrak{s p}(2 \ell)$ be the Lie algebra of $\operatorname{Sp}(2 \ell)$. We take as a Borel subalgebra of $\mathfrak{s p}(2 \ell)$ the subalgebra of upper triangular matrices, and as a Cartan subalgebra $H$ the subalgebra spanned by $\tilde{e}_{i}=e_{i i}-e_{2 \ell+1-i, 2 \ell+1-i}(1 \leq i \leq \ell)$. A finite-dimensional irreducible representation of $\mathfrak{s p}(2 \ell)$ is determined by its highest weight $\lambda \in H^{*}$ with $\left\langle\lambda, \tilde{e}_{i}\right\rangle=\lambda_{i} \in \mathbb{Z}_{+}(1 \leq i \leq \ell)$ and $\lambda_{1} \geq \ldots \geq \lambda_{\ell}$. Furthermore each such representation lifts to an irreducible representation of $\operatorname{Sp}(2 \ell)$, which is denoted by $V_{\mathrm{Sp}(2 \ell)}^{\lambda}$. We identify $\lambda$ with $\left(\lambda_{1}, \ldots, \lambda_{\ell}\right)$, and put

$$
\mathcal{P}(\operatorname{Sp}(2 \ell)):=\left\{\lambda=\left(\lambda_{1}, \cdots, \lambda_{\ell}\right) \mid \lambda_{i} \in \mathbb{Z}_{+}, \lambda_{1} \geq \ldots \geq \lambda_{\ell}\right\},
$$

which is the set of partitions of length no more than $\ell$.

Proposition 2.2. [W, Theorem 3.4] There exists an action of $\mathfrak{c}_{\infty} \times \operatorname{Sp}(2 \ell)$ on $\mathfrak{F}^{\ell}$. Furthermore, under this joint action, we have

$$
\mathfrak{F}^{\ell} \cong \bigoplus_{\lambda \in \mathcal{P}(\operatorname{Sp}(2 \ell))} L\left(\mathfrak{c}_{\infty}, \Lambda^{\mathfrak{c}}(\lambda)\right) \otimes V_{\mathrm{Sp}(2 \ell)}^{\lambda}
$$

where $\Lambda^{\mathfrak{c}}(\lambda)=\ell \Lambda_{0}^{\mathfrak{c}}+\sum_{k \geq 1} \lambda_{k}^{\prime} \epsilon_{k}=(\ell-j) \Lambda_{0}^{\mathfrak{c}}+\sum_{k=1}^{j} \Lambda_{\lambda_{k}}^{\mathfrak{c}}$ and $j$ is the number of non-zero parts of $\lambda$.

Computing the trace of the operator $\prod_{n \in \mathbb{N}} x_{n}^{\widetilde{E}_{n}} \prod_{i=1}^{\ell} z_{i}^{\tilde{e}_{i}}$ on both sides of (2.7), we obtain the following identity:

$$
\prod_{i=1}^{\ell} \prod_{n \in \mathbb{N}}\left(1+x_{n} z_{i}\right)\left(1+x_{n} z_{i}^{-1}\right)=\sum_{\lambda \in \mathcal{P}(\operatorname{Sp}(2 \ell))} \operatorname{ch} L\left(\mathfrak{c}_{\infty}, \Lambda^{\mathfrak{c}}(\lambda)\right) \operatorname{ch} V_{\operatorname{Sp}(2 \ell)}^{\lambda} .
$$

2.3.3. The $\left(\mathfrak{d}_{\infty}, \mathrm{O}(m)\right)$-duality. Let $m$ be a positive integer greater than 1 . We define $\ell$ by $m=2 \ell$ when $m$ is even, and $m=2 \ell+1$ when $m$ is odd. Let $\mathrm{O}(m)$ denote the orthogonal group which is the subgroup of $\mathrm{GL}(m)$ preserving the non-degenerate symmetric bilinear form on $\mathbb{C}^{m}$ determined by $J_{m}$ of $(2.6)$. Let $\mathfrak{s o}(m)$ be the Lie algebra of $\mathrm{O}(m)$. We take as a Cartan subalgebra $H$ of $\mathfrak{s o}(m)$ the subalgebra spanned by $\tilde{e}_{i}=e_{i i}-e_{m+1-i, m+1-i}(1 \leq i \leq \ell)$, while we take as the Borel subalgebra the subalgebra of upper triangular matrices.

For $\lambda \in H^{*}$ let us identify $\lambda$ with $\left(\lambda_{1}, \ldots, \lambda_{\ell}\right)$, where $\lambda_{i}=\left\langle\lambda, \tilde{e}_{i}\right\rangle$ for $1 \leq i \leq \ell$. Then a finite-dimensional irreducible representation of $\mathfrak{s o}(2 \ell)$ is determined by its highest weight $\lambda$ satisfying the condition $\lambda_{1} \geq \ldots \geq \lambda_{\ell-1} \geq\left|\lambda_{\ell}\right|$ with either $\lambda_{i} \in \mathbb{Z}$ or else $\lambda_{i} \in \frac{1}{2}+\mathbb{Z}$, for $1 \leq i \leq \ell$. Furthermore it lifts to a representation of $\mathrm{SO}(2 \ell)$ if and only if $\lambda_{i} \in \mathbb{Z}$ for $1 \leq i \leq \ell$. Also a finite-dimensional irreducible representation of $\mathfrak{s o}(2 \ell+1)$ is determined by its highest weight $\lambda$ satisfying the conditions $\lambda_{1} \geq \ldots \geq \lambda_{\ell}$ with either $\lambda_{i} \in \mathbb{Z}_{+}$or else $\lambda_{i} \in \frac{1}{2}+\mathbb{Z}_{+}$, for $1 \leq i \leq \ell$. Furthermore it lifts to a representation of $\mathrm{SO}(2 \ell+1)$ if and only if $\lambda_{i} \in \mathbb{Z}_{+}$for $1 \leq i \leq \ell$ 
We put

$$
\mathcal{P}(\mathrm{O}(m)):=\left\{\lambda=\left(\lambda_{1}, \cdots, \lambda_{m}\right) \mid \lambda_{i} \in \mathbb{Z}_{+}, \lambda_{1} \geq \ldots \geq \lambda_{m}, \lambda_{1}^{\prime}+\lambda_{2}^{\prime} \leq m\right\} .
$$

For $\lambda \in \mathcal{P}(\mathrm{O}(m))$, let $\tilde{\lambda}$ be the partition obtained from $\lambda$ by replacing its first column with $m-\lambda_{1}^{\prime}$.

Suppose that $m=2 \ell$ and $\lambda=\left(\lambda_{1}, \ldots, \lambda_{\ell}, 0, \ldots, 0\right) \in \mathcal{P}(\mathrm{O}(2 \ell))$ is given. If $\lambda_{\ell}>0$, let $V_{\mathrm{O}(2 \ell)}^{\lambda}$ be the irreducible $\mathrm{O}(2 \ell)$-module, which as an $\mathfrak{s o}(2 \ell)$-module, is isomorphic to the direct sum of irreducible representations of highest weights $\left(\lambda_{1}, \ldots, \lambda_{\ell}\right)$ and $\left(\lambda_{1}, \ldots,-\lambda_{\ell}\right)$. If $\lambda_{\ell}=0$, let $V_{\mathrm{O}(2 \ell)}^{\lambda}$ denote the $\mathrm{O}(2 \ell)$-module that as an $\mathfrak{s o}(2 \ell)$-module is isomorphic to the irreducible representation of highest weight $\left(\lambda_{1}, \cdots, \lambda_{\ell-1}, 0\right)$, and on which the element $\tau=\sum_{i \neq \ell, \ell+1} e_{i i}+e_{\ell, \ell+1}+e_{\ell+1, \ell} \in \mathrm{O}(2 \ell) \backslash \mathrm{SO}(2 \ell)$ transforms trivially on highest weight vectors. Set $V_{\mathrm{O}(2 \ell)}^{\tilde{\lambda}}=V_{\mathrm{O}(2 \ell)}^{\lambda} \otimes$ det, where det is the onedimensional non-trivial representation of $\mathrm{O}(2 \ell)$.

Suppose that $m=2 \ell+1$ and $\lambda=\left(\lambda_{1}, \ldots, \lambda_{\ell}, 0, \ldots, 0\right) \in \mathcal{P}(\mathrm{O}(m))$ is given. Let $V_{\mathrm{O}(2 \ell+1)}^{\lambda}$ be the irreducible $\mathrm{O}(2 \ell+1)$-module isomorphic to the irreducible representation of highest weight $\left(\lambda_{1}, \ldots, \lambda_{\ell}\right)$ as an $\mathfrak{s o}(2 \ell+1)$-module, on which $-I_{m}$ acts trivially. Here $I_{m}$ is the $m \times m$ identity matrix. Also, we let $V_{\mathrm{O}(2 \ell+1)}^{\tilde{\lambda}}=V_{\mathrm{O}(2 \ell+1)}^{\lambda} \otimes$ det (cf. e.g. [BT, H1] for more details).

Proposition 2.3. [W, Theorems 3.2 and 4.1] There exists an action of $\mathfrak{d}_{\infty} \times \mathrm{O}(m)$ on $\mathfrak{F}^{\frac{m}{2}}$. Furthermore under this joint action we have

$$
\mathfrak{F}^{\frac{m}{2}} \cong \bigoplus_{\lambda \in \mathcal{P}(\mathrm{O}(m))} L\left(\mathfrak{d}_{\infty}, \Lambda^{\mathfrak{d}}(\lambda)\right) \otimes V_{\mathrm{O}(m)}^{\lambda}
$$

where $\Lambda^{\mathfrak{d}}(\lambda)=m \Lambda_{0}^{\mathfrak{d}}+\sum_{k \geq 1} \lambda_{k}^{\prime} \epsilon_{k}$.

Suppose that $m=2 \ell$. Computing the trace of the operator $\prod_{n \in \mathbb{N}} x_{n}^{\widetilde{E}_{n}} \prod_{i=1}^{\ell} z_{i}^{\tilde{e}_{i}}$ on both sides of (2.9), we obtain

$$
\prod_{i=1}^{\ell} \prod_{n \in \mathbb{N}}\left(1+x_{n} z_{i}\right)\left(1+x_{n} z_{i}^{-1}\right)=\sum_{\lambda \in \mathcal{P}(\mathrm{O}(2 \ell))} \operatorname{ch} L\left(\mathfrak{d}_{\infty}, \Lambda^{\mathfrak{d}}(\lambda)\right) \operatorname{ch} V_{\mathrm{O}(2 \ell)}^{\lambda} .
$$

Suppose that $m=2 \ell+1$. Let $\epsilon$ be the eigenvalue of $-I_{m}$ on $\mathrm{O}(2 \ell+1)$-modules satisfying $\epsilon^{2}=1$. From the computation of the trace of $\prod_{n \in \mathbb{N}} x_{n}^{\widetilde{E}_{n}} \prod_{i=1}^{\ell} z_{i}^{\tilde{e}_{i}}\left(-I_{m}\right)$ on both sides of (2.9), we obtain

$$
\prod_{i=1}^{\ell} \prod_{n \in \mathbb{N}}\left(1+\epsilon x_{n} z_{i}\right)\left(1+\epsilon x_{n} z_{i}^{-1}\right)\left(1+\epsilon x_{n}\right)=\sum_{\lambda \in \mathcal{P}(\mathrm{O}(2 \ell+1))} \operatorname{ch} L\left(\mathfrak{d}_{\infty}, \Lambda^{\mathfrak{d}}(\lambda)\right) \operatorname{ch} V_{\mathrm{O}(2 \ell+1)}^{\lambda}
$$

Note that $\operatorname{ch} V_{\mathrm{O}(2 \ell)}^{\lambda}$ is a Laurent polynomial in $z_{1}, \ldots, z_{\ell}$ and $\operatorname{ch} V_{\mathrm{O}(2 \ell)}^{\lambda}=\operatorname{ch} V_{\mathrm{O}(2 \ell)}^{\tilde{\lambda}}$, while $\operatorname{ch} V_{\mathrm{O}(2 \ell+1)}^{\lambda}$ is the Laurent polynomial in $z_{1}, \ldots, z_{\ell}, \epsilon$ and $\operatorname{ch} V_{\mathrm{O}(2 \ell+1)}^{\lambda}=\epsilon \operatorname{ch} V_{\mathrm{O}(2 \ell+1)}^{\tilde{\lambda}}$. 
2.3.4. The $\left(\mathfrak{b}_{\infty}, \operatorname{Pin}(2 \ell)\right)$-duality. The Lie group $\operatorname{Pin}(2 \ell)$ is a double cover of $\mathrm{O}(2 \ell)$, with $\operatorname{Spin}(2 \ell)$ as the inverse image of $\mathrm{SO}(2 \ell)$ under the covering map (see e.g. [BT]). An irreducible representation of $\operatorname{Spin}(2 \ell)$ that does not factor through $\mathrm{SO}(2 \ell)$ is an irreducible representation of $\mathfrak{s o}(2 \ell)$ of highest weight of the form

$$
\left(\lambda_{1}+\frac{1}{2}, \ldots, \lambda_{\ell-1}+\frac{1}{2}, \lambda_{\ell}+\frac{1}{2}\right), \text { or }\left(\lambda_{1}+\frac{1}{2}, \ldots, \lambda_{\ell-1}+\frac{1}{2},-\lambda_{\ell}-\frac{1}{2}\right),
$$

where $\lambda_{1} \geq \ldots \geq \lambda_{\ell}$ with $\lambda_{i} \in \mathbb{Z}_{+}$for $1 \leq i \leq \ell$. We put

$$
\mathcal{P}(\operatorname{Pin}(2 \ell)):=\left\{\lambda=\left(\lambda_{1}, \cdots, \lambda_{\ell}\right) \mid \lambda_{i} \in \mathbb{Z}_{+}, \lambda_{1} \geq \ldots \geq \lambda_{\ell}\right\} .
$$

For $\lambda \in \mathcal{P}(\operatorname{Pin}(2 \ell))$, let us denote by $V_{\operatorname{Pin}(2 \ell)}^{\lambda}$ the irreducible representation of $\operatorname{Pin}(2 \ell)$ induced from the irreducible representation of $\operatorname{Spin}(2 \ell)$ whose highest weight is given by either of the two weights in (2.12). When restricted to $\operatorname{Spin}(2 \ell), V_{\operatorname{Pin}(2 \ell)}^{\lambda}$ decomposes into a direct sum of two irreducible representations of highest weights given by those in (2.12).

Proposition 2.4. [W, Theorem 3.3] There exists an action of $\mathfrak{b}_{\infty} \times \operatorname{Pin}(2 \ell)$ on $\mathfrak{F}^{\ell}$. Furthermore, under this joint action, we have

$$
\mathfrak{F}^{\ell} \cong \bigoplus_{\lambda \in \mathcal{P}(\operatorname{Pin}(2 \ell))} L\left(\mathfrak{b}_{\infty}, \Lambda^{\mathfrak{b}}(\lambda)\right) \otimes V_{\operatorname{Pin}(2 \ell)}^{\lambda},
$$

where $\Lambda^{\mathfrak{b}}(\lambda)=2 \ell \Lambda_{0}^{\mathfrak{b}}+\sum_{k \geq 1} \lambda_{k}^{\prime} \epsilon_{k}$.

Taking the trace of the operator $\prod_{n \in \mathbb{N}} x_{n}^{\widetilde{E}_{n}} \prod_{i=1}^{\ell} z_{i}^{\tilde{e}_{i}}$ on both sides of (2.13), gives

$$
\prod_{i=1}^{\ell} \prod_{n \in \mathbb{N}}\left(z_{i}^{\frac{1}{2}}+z_{i}^{-\frac{1}{2}}\right)\left(1+x_{n} z_{i}\right)\left(1+x_{n} z_{i}^{-1}\right)=\sum_{\lambda \in \mathcal{P}(\operatorname{Pin}(2 \ell))} \operatorname{ch} L\left(\mathfrak{b}_{\infty}, \Lambda^{\mathfrak{b}}(\lambda)\right) \operatorname{ch} V_{\operatorname{Pin}(2 \ell)}^{\lambda} .
$$

In what follows we mean by $(\mathfrak{g}, G)$ one of the dual pairs of Section 2.3, and by $\mathfrak{x} \in\{\mathfrak{a}, \mathfrak{b}, \mathfrak{c}, \mathfrak{d}\}$ the type of $\mathfrak{g}$.

2.4. $\mathfrak{u}_{-}$-homology groups of $\mathfrak{g}$-modules. Let $\Delta:=\Delta^{+} \cup \Delta^{-}$be the set of roots of $\mathfrak{g}$, where $\Delta^{-}=-\Delta^{+}$. Let $\Delta_{S}^{ \pm}:=\Delta^{ \pm} \cap\left(\sum_{j \neq 0} \mathbb{Z} \alpha_{j}\right)$ and $\Delta^{ \pm}(S):=\Delta^{ \pm} \backslash \Delta_{S}^{ \pm}$. Denote by $\mathfrak{g}_{\alpha}$ the root space corresponding to $\alpha \in \Delta$. Set

$$
\mathfrak{u}_{ \pm}:=\sum_{\alpha \in \Delta^{ \pm}(S)} \mathfrak{g}_{\alpha}, \quad \mathfrak{l}:=\sum_{\alpha \in \Delta_{S}^{+} \cup \Delta_{S}^{-}} \mathfrak{g}_{\alpha} \oplus \mathfrak{h} .
$$

Then we have $\mathfrak{g}=\mathfrak{u}_{+} \oplus \mathfrak{l} \oplus \mathfrak{u}_{-}$. The Lie algebras $\mathfrak{l}$ and $\mathfrak{g}$ share the same Cartan subalgebra $\mathfrak{h}$. For $\mu \in \mathfrak{h}^{*}$ we denote by $L(\mathfrak{l}, \mu)$ the irreducible highest weight representation of $\mathfrak{l}$ with highest weight $\mu$. For $\mathfrak{g}=\widehat{\mathfrak{g l}}_{\infty}$ we denote by $\mathcal{P}_{\mathfrak{l}}^{+}$the set of $\mu=\sum_{i \in \mathbb{Z}} \mu_{i} \epsilon_{i}+c \Lambda_{0}^{\mathfrak{a}} \in \mathfrak{h}^{*}$ with $\left(\mu_{1}, \mu_{2}, \cdots\right) \in \mathcal{P}^{+},\left(-\mu_{0},-\mu_{-1}, \cdots\right) \in \mathcal{P}^{+}$, and $c \in \mathbb{C}$. For $\mathfrak{g}=\mathfrak{b}_{\infty}, \mathfrak{c}_{\infty}, \mathfrak{d}_{\infty}$, let $\mathcal{P}_{\mathfrak{l}}^{+}$denote the set of $\mu=\sum_{i \in \mathbb{N}} \mu_{i} \epsilon_{i}+c \Lambda_{0}^{x} \in \mathfrak{h}^{*}$ with $\left(\mu_{1}, \mu_{2}, \cdots\right) \in \mathcal{P}^{+}$and $c \in \mathbb{C}$. 
Let $V$ be a $\mathfrak{g}$-module and let $C_{k}\left(\mathfrak{u}_{-} ; V\right):=\Lambda^{k}\left(\mathfrak{u}_{-}\right) \otimes V$ be the space of the $k$-th chains $\left(k \in \mathbb{Z}_{+}\right)$. Denote the boundary operator by $d_{k}: C_{k}\left(\mathfrak{u}_{-} ; V\right) \rightarrow C_{k-1}\left(\mathfrak{u}_{-} ; V\right)$ (see e.g. GL, J, L]). We have a complex

$$
\cdots \stackrel{d_{k+1}}{\longrightarrow} C_{k}\left(\mathfrak{u}_{-} ; V\right) \stackrel{d_{k}}{\longrightarrow} C_{k-1}\left(\mathfrak{u}_{-} ; V\right) \stackrel{d_{k-1}}{\longrightarrow} \cdots \stackrel{d_{2}}{\longrightarrow} \mathfrak{u}_{-} \otimes V \stackrel{d_{1}}{\longrightarrow} V \stackrel{d_{0}}{\longrightarrow} 0
$$

with $d_{k} d_{k+1}=0$ and homology groups $\mathrm{H}_{k}\left(\mathfrak{u}_{-} ; V\right):=\operatorname{ker} d_{k} / \operatorname{im} d_{k+1}$ for $k \in \mathbb{Z}_{+}$.

The subalgebra $\mathfrak{l}$ acts on $V$ by restriction, while it acts on $\mathfrak{u}_{-}$via the adjoint action. Thus $\mathfrak{l}$ acts on the chains. Furthermore the $\mathfrak{l}$-action commutes with boundary operators and hence the homology group $\mathrm{H}_{k}\left(\mathfrak{u}_{-} ; V\right)$ is an $\mathfrak{l}$-module, for all $k \in \mathbb{Z}_{+}$. In order to describe these groups in the case when $V=L\left(\mathfrak{g}, \Lambda^{\mathfrak{x}}(\lambda)\right)$, for $\lambda \in \mathcal{P}(G)$, we introduce further notation.

For $j \in I$, define simple reflections $\sigma_{j}$ by

$$
\sigma_{j}(\mu):=\mu-\left\langle\mu, \alpha_{j}^{\vee}\right\rangle \alpha_{j}
$$

where $\mu \in \mathfrak{h}^{*}$. Let $W$ be the subgroup of $\operatorname{Aut}\left(\mathfrak{h}^{*}\right)$ generated by the simple reflections, i.e. $W$ is the Weyl group of $\mathfrak{g}$. For each $w \in W$ we let $l(w)$ denote the length of $w$. We have an action on $\mathfrak{h}$ given by $\sigma_{j}(h)=h-\left\langle\alpha_{j}, h\right\rangle \alpha_{j}^{\vee}$ for $h \in \mathfrak{h}$ so that $\langle w(\mu), w(h)\rangle=\langle\mu, h\rangle$, for $w \in W, \mu \in \mathfrak{h}^{*}$ and $h \in \mathfrak{h}$. We also define

$$
w \circ \mu:=w\left(\mu+\rho_{c}\right)-\rho_{c}, \quad \mu \in \mathfrak{h}^{*}, w \in W .
$$

Consider $W_{0}$ the subgroup of $W$ generated by $\sigma_{j}$ with $j \neq 0$. Let

$$
W^{0}:=\left\{w \in W \mid w\left(\Delta^{-}\right) \cap \Delta^{+} \subseteq \Delta^{+}(S)\right\} .
$$

It is well-known that $W=W_{0} W^{0}$ and $W^{0}$ is the set of the minimal length representatives of the right coset space $W_{0} \backslash W$ (cf. $[\mathrm{L}]$ ). For $k \in \mathbb{Z}_{+}$, set

$$
W_{k}^{0}:=\left\{w \in W^{0} \mid l(w)=k\right\} .
$$

Given $\lambda \in \mathcal{P}(G)$ it is easy to see that $\left\langle\Lambda^{\mathfrak{x}}(\lambda), \alpha_{j}^{\vee}\right\rangle \in \mathbb{Z}_{+}$, for all $j \in I$. Since $w \in W^{0}$ implies that $w^{-1}\left(\Delta_{S}^{+}\right) \subseteq \Delta^{+}$, we obtain $\left\langle w \circ \Lambda^{\mathfrak{x}}(\lambda), \alpha_{j}^{\vee}\right\rangle \in \mathbb{Z}_{+}$, for all $j \in I \backslash\{0\}$. Since $\mathfrak{g}$ is a generalized Kac-Moody algebra and each $L\left(\mathfrak{g}, \Lambda^{\mathfrak{x}}(\lambda)\right)$ is an integrable (=standard) module, the description of the l-module structure of the homology groups $\mathrm{H}_{k}\left(\mathfrak{u}_{-} ; L\left(\mathfrak{g}, \Lambda^{\mathfrak{x}}(\lambda)\right)\right.$ in [J] remains valid. It is given as follows.

Proposition 2.5. [J] Let $\lambda \in \mathcal{P}(G)$ and $k \in \mathbb{Z}_{+}$. We have, as $\mathfrak{l}$-modules,

$$
\mathrm{H}_{k}\left(\mathfrak{u}_{-} ; L\left(\mathfrak{g}, \Lambda^{\mathfrak{x}}(\lambda)\right)\right) \cong \bigoplus_{w \in W_{k}^{0}} L\left(\mathfrak{l}, w \circ \Lambda^{\mathfrak{x}}(\lambda)\right) .
$$

We may write $w \circ \Lambda^{\mathfrak{x}}(\lambda)$ as

$$
w \circ \Lambda^{\mathfrak{x}}(\lambda)= \begin{cases}\sum_{j>0}\left(\lambda_{w}^{+}\right)_{j} \epsilon_{j}-\sum_{j \geq 0}\left(\lambda_{w}^{-}\right)_{j+1} \epsilon_{-j}+n \Lambda_{0}^{\mathfrak{a}}, & \text { if } \mathfrak{x}=\mathfrak{a}, \\ \sum_{j>0}\left(\lambda_{w}\right)_{j} \epsilon_{j}+n \Lambda_{0}^{\mathfrak{x}}, & \text { otherwise }\end{cases}
$$


where $n=\left\langle\Lambda^{\mathfrak{x}}(\lambda), K\right\rangle /\left\langle\Lambda_{0}^{\mathfrak{x}}, K\right\rangle$. Furthermore, $\lambda_{w}^{ \pm}=\left(\left(\lambda_{w}^{ \pm}\right)_{1},\left(\lambda_{w}^{ \pm}\right)_{2}, \ldots\right)$ and $\lambda_{w}=$ $\left(\left(\lambda_{w}\right)_{1},\left(\lambda_{w}\right)_{2}, \ldots\right)$ are partitions. Thus, suppressing the action of $K$, we see that the character of the l-module $\mathrm{H}_{k}\left(\mathfrak{u}_{-} ; L\left(\mathfrak{g}, \Lambda^{\mathfrak{x}}(\lambda)\right)\right)$ is

$$
\operatorname{chH}_{k}\left(\mathfrak{u}_{-} ; L\left(\mathfrak{g}, \Lambda^{\mathfrak{x}}(\lambda)\right)\right)= \begin{cases}\sum_{w \in W_{k}^{0}} s_{\lambda_{w}^{+}}\left(x_{1}, x_{2}, \cdots\right) s_{\lambda_{w}^{-}}\left(x_{0}^{-1}, x_{-1}^{-1}, \cdots\right), & \text { if } \mathfrak{x}=\mathfrak{a} \\ \sum_{w \in W_{k}^{0}} s_{\lambda_{w}}\left(x_{1}, x_{2}, \cdots\right), & \text { otherwise }\end{cases}
$$

where here and further $s_{\mu}\left(y_{1}, y_{2}, \cdots\right)$ denotes the Schur function in the variables $y_{1}, y_{2}, \cdots$ associated to the partition $\mu$. Now applying the Euler-Poincaré principle to (2.16) one obtains

$$
\sum_{k=0}^{\infty}(-1)^{k} \operatorname{ch} C_{k}\left(\mathfrak{u}_{-} ; L\left(\mathfrak{g}, \Lambda^{\mathfrak{x}}(\lambda)\right)\right)=\sum_{k=0}^{\infty}(-1)^{k} \operatorname{chH}_{k}\left(\mathfrak{u}_{-} ; L\left(\mathfrak{g}, \Lambda^{\mathfrak{x}}(\lambda)\right)\right) .
$$

Since

$$
\sum_{k=0}^{\infty}(-1)^{k} \operatorname{ch} C_{k}\left(\mathfrak{u}_{-} ; L\left(\mathfrak{g}, \Lambda^{\mathfrak{x}}(\lambda)\right)\right)=\operatorname{ch} L\left(\mathfrak{g}, \Lambda^{\mathfrak{x}}(\lambda)\right) D^{\mathfrak{x}}
$$

where

$$
D^{\mathfrak{x}}= \begin{cases}\prod_{i, j}\left(1-x_{-i+1}^{-1} x_{j}\right), & \text { if } \mathfrak{x}=\mathfrak{a} \\ \prod_{i}\left(1-x_{i}\right) \prod_{i<j}\left(1-x_{i} x_{j}\right), & \text { if } \mathfrak{x}=\mathfrak{b} \\ \prod_{i}\left(1-x_{i}^{2}\right) \prod_{i<j}\left(1-x_{i} x_{j}\right), & \text { if } \mathfrak{x}=\mathfrak{c} \\ \prod_{i<j}\left(1-x_{i} x_{j}\right), & \text { if } \mathfrak{x}=\mathfrak{d}\end{cases}
$$

with $i, j \in \mathbb{N}$, we obtain

$$
\operatorname{ch} L\left(\mathfrak{g}, \Lambda^{\mathfrak{x}}(\lambda)\right)=\frac{1}{D^{\mathfrak{x}}} \sum_{k=0}^{\infty}(-1)^{k} \operatorname{chH}_{k}\left(\mathfrak{u}_{-} ; L\left(\mathfrak{g}, \Lambda^{\mathfrak{x}}(\lambda)\right)\right) .
$$

\section{INFINITE-DIMENSIONAL LIE SUPERALGEBRAS}

3.1. The Lie superalgebra $\widehat{\mathfrak{g l}}_{\infty \mid \infty}$ and its subalgebras of classical types. Let us recall the infinite-dimensional Lie superalgebra $\widehat{\mathfrak{g l}}_{\infty \mid \infty}$ and the subalgebras $\widehat{\mathcal{B}}$, $\widehat{\mathfrak{C}}, \widehat{\mathcal{D}}$ of classical types. We will say that these superalgebras are of types $\mathfrak{a}, \mathfrak{b}, \mathfrak{c}, \mathfrak{d}$, respectively. The following notation will also be assumed throughout the paper.

$\cdot \overline{\mathfrak{g}}$ : the Lie superalgebra $\widehat{\mathfrak{g l}}_{\infty \mid \infty}$, or its subalgebra $\widehat{\mathcal{B}}, \widehat{\mathrm{C}}, \widehat{\mathcal{D}}$,

$\cdot \overline{\mathfrak{h}}$ : a Cartan subalgebra of $\overline{\mathfrak{g}}$,

$\cdot \bar{I}$ : the index set for simple roots,

$\cdot \bar{\Pi}=\left\{\beta_{r} \mid r \in \bar{I}\right\}:$ the set of simple roots,

$\cdot \bar{\Pi}^{\vee}=\left\{\beta_{r}^{\vee} \mid r \in \bar{I}\right\}$ : the set of simple coroots,

$\cdot \bar{\Delta}^{+}$: the set of positive roots. 
3.1.1. The Lie superalgebra $\widehat{\mathfrak{g l}}_{\infty \mid \infty}$. Let $\left.\mathbb{C}^{\infty}\right|_{\infty}$ be the infinite-dimensional superspace over $\mathbb{C}$ with a basis $\left\{e_{r} \mid r \in \frac{1}{2} \mathbb{Z}\right\}$. We assume that $\operatorname{deg} e_{r}=\overline{0}$, for $r \in \mathbb{Z}$, and $\operatorname{deg} e_{r}=\overline{1}$, otherwise. We may identify $\operatorname{End}\left(\mathbb{C}^{\infty} \mid \infty\right)$ with the Lie superalgebra of matrices $\left(a_{r s}\right)\left(r, s \in \frac{1}{2} \mathbb{Z}\right)$. Let $\mathfrak{g l}_{\infty \mid \infty}$ denote the subalgebra consisting of $\left(a_{r s}\right)$ with $a_{r s}=0$ for all but finitely many $a_{r s}$ 's. Denote by $E_{r s}$ the elementary matrix with 1 at the $r$-th row and $s$-th column and zero elsewhere. Denote by $\widehat{\mathfrak{g l}}_{\infty \mid \infty}=\mathfrak{g l}_{\infty \mid \infty} \oplus \mathbb{C} K$ the central extension of $\mathfrak{g l}_{\infty \mid \infty}$ by a one-dimensional center $\mathbb{C} K$ given by the 2 -cocycle

$$
\beta(A, B):=\operatorname{Str}([\bar{J}, A] B),
$$

where $\bar{J}=\sum_{r \leq 0} E_{r r}$. Here, for a matrix $D=\left(d_{r s}\right)$, the supertrace is defined by $\operatorname{Str} D=\sum_{r \in \frac{1}{2} \mathbb{Z}}(-1)^{2 r} d_{r r}$. Then $\overline{\mathfrak{h}}=\sum_{r \in \frac{1}{2} \mathbb{Z}} \mathbb{C} E_{r r} \oplus \mathbb{C} K$ is a Cartan subalgebra. Put $\bar{I}=\frac{1}{2} \mathbb{Z}$. We have

$$
\begin{aligned}
\bar{\Pi}^{\vee} & =\left\{\beta_{r}^{\vee}=E_{r r}+E_{r+\frac{1}{2}, r+\frac{1}{2}}+\delta_{r 0} K(r \in \bar{I})\right\}, \\
\bar{\Pi} & =\left\{\beta_{r}=\delta_{r}-\delta_{r+\frac{1}{2}}(r \in \bar{I})\right\}, \\
\bar{\Delta}^{+} & =\left\{\delta_{r}-\delta_{s}(r, s \in \bar{I}, r<s)\right\},
\end{aligned}
$$

where $\delta_{r} \in \overline{\mathfrak{h}}^{*}$ is determined by $\left\langle\delta_{r}, E_{s s}\right\rangle=\delta_{r s}$ and $\left\langle\delta_{r}, K\right\rangle=0$.

By assigning degree 0 to the Cartan subalgebra and setting $\operatorname{deg} E_{r s}=s-r$, we equip $\widehat{\mathfrak{g l}}_{\infty \mid \infty}$ with a $\frac{1}{2} \mathbb{Z}$-gradation: $\widehat{\mathfrak{g l}}_{\infty \mid \infty}=\bigoplus_{k \in \frac{1}{2} \mathbb{Z}}\left(\widehat{\mathfrak{g l}}_{\infty \mid \infty}\right)_{k}$. This leads to the triangular decomposition:

$$
\widehat{\mathfrak{g l}}_{\infty \mid \infty}=\left(\widehat{\mathfrak{g l}}_{\infty \mid \infty}\right)_{+} \oplus\left(\widehat{\mathfrak{g l}}_{\infty \mid \infty}\right)_{0} \oplus\left(\widehat{\mathfrak{g l}}_{\infty \mid \infty}\right)_{-},
$$

where $\left(\widehat{\mathfrak{g l}}_{\infty \mid \infty}\right)_{ \pm}=\bigoplus_{k \in \pm \frac{1}{2} \mathbb{N}}\left(\widehat{\mathfrak{g l}}_{\infty \mid \infty}\right)_{k}$ and $\left(\widehat{\mathfrak{g l}}_{\infty \mid \infty}\right)_{0}=\overline{\mathfrak{h}}$.

3.1.2. The Lie superalgebras $\widehat{\mathcal{B}}, \widehat{\mathcal{C}}, \widehat{\mathcal{D}}$. Below we follow the presentations of [CW2, LZ]. Let $L=L_{\overline{0}} \oplus L_{\overline{1}}$ be a Lie superalgebra, which is a subalgebra of $\mathfrak{g l}_{\infty \mid \infty}$. We say that $L$ preserves a bilinear form $(\cdot \mid \cdot)$ on $\mathbb{C}^{\infty / \infty}$ if

$$
L_{\epsilon}=\left\{A \in\left(\widehat{\mathfrak{g l}}_{\infty \mid \infty}\right)_{\epsilon}\left|(A v \mid w)=-(-1)^{\epsilon|v|}(v \mid A w), v, w \in \mathbb{C}^{\infty}\right| \infty\right\}, \quad \epsilon=\overline{0}, \overline{1},
$$

where $|v|$ denotes the parity of $v$ in $\mathbb{C}^{\infty} \mid \infty$ (cf. [K1]).

First we define the Lie superalgebra $\mathcal{B}$ to be the subalgebra of $\mathfrak{g l}_{\infty \mid \infty}$ preserving the following super-symmetric bilinear form on $\mathbb{C}^{\infty} \mid \infty$ :

$$
\begin{aligned}
& \left(e_{i} \mid e_{j}\right)=(-1)^{i} \delta_{i,-j}, \quad i, j \in \mathbb{Z}, \\
& \left(e_{r} \mid e_{s}\right)=(-1)^{r+\frac{1}{2}} \delta_{r,-s}, \quad r, s \in \frac{1}{2}+\mathbb{Z}, \\
& \left(e_{i} \mid e_{r}\right)=0, \quad i \in \mathbb{Z}, r \in \frac{1}{2}+\mathbb{Z} .
\end{aligned}
$$

Let $\mathcal{A}$ be the subalgebra of $\mathfrak{g l}_{\infty \mid \infty}$ consisting of matrices $A=\left(a_{i j}\right)$ such that $a_{i j}=0$ if $i=0$ or $j=0$. We define the Lie superalgebras $\mathcal{C}$ and $\mathcal{D}$ to be the 
subalgebras of $\mathcal{A}$ preserving the following super-skew-symmetric bilinear forms on $\left.\mathbb{C}^{\infty}\right|^{\infty}$, respectively:

$$
\begin{aligned}
& \left(e_{i} \mid e_{j}\right)=\left\{\begin{array}{ll}
\operatorname{sgn}(i) \delta_{i,-j}, & \text { for } \mathcal{C}, \\
\delta_{i,-j}, & \text { for } \mathcal{D},
\end{array} \quad i, j \in \mathbb{Z} \backslash\{0\},\right. \\
& \left(e_{r} \mid e_{s}\right)=\left\{\begin{array}{ll}
\delta_{r,-s}, & \text { for } \mathcal{C}, \\
\operatorname{sgn}(r) \delta_{r,-s}, & \text { for } \mathcal{D},
\end{array} \quad r, s \in \frac{1}{2}+\mathbb{Z},\right. \\
& \left(e_{i} \mid e_{r}\right)=0, \quad i \in \mathbb{Z}, r \in \frac{1}{2}+\mathbb{Z} .
\end{aligned}
$$

Now let $X$ be one of $\mathcal{B}, \mathcal{C}, \mathcal{D}$. We define $\widehat{x}$ to be the central extension of $x$ given by the restriction of the two-cocycle (3.1). Then $\widehat{x}$ has a natural triangular decomposition induced from $\widehat{\mathfrak{g l}}_{\infty \mid \infty}$ :

$$
\widehat{x}=\widehat{x}_{+} \oplus \widehat{x}_{0} \oplus \widehat{x}_{-}
$$

where $\widehat{X}_{0}=\overline{\mathfrak{h}}=\sum_{r \in \frac{1}{2} \mathbb{N}} \mathbb{C} \widetilde{E}_{r} \oplus \mathbb{C} K$ is a Cartan subalgebra, with $\widetilde{E}_{r}=E_{r r}-E_{-r,-r}$ for $r \in \frac{1}{2} \mathbb{N}$. We may regard $\delta_{r} \in\left(\widehat{\mathfrak{g l}}_{\infty \mid \infty}\right)_{0}^{*}$ as an element in $\widehat{X}_{0}^{*}$ via restriction so that $\left\langle\delta_{r}, \widetilde{E}_{s}\right\rangle=\delta_{r s}$ for $r, s \in \frac{1}{2} \mathbb{N}$. Put $\bar{I}=\frac{1}{2} \mathbb{Z}_{+}$. Then we have

- $\widehat{\mathcal{B}}$

$$
\begin{aligned}
\bar{\Pi}^{\vee} & =\left\{\beta_{0}^{\vee}=\widetilde{E}_{\frac{1}{2}}+K, \beta_{r}^{\vee}=\widetilde{E}_{r}+\widetilde{E}_{r+\frac{1}{2}}\left(r \in \frac{1}{2} \mathbb{N}\right)\right\}, \\
\bar{\Pi} & =\left\{\beta_{0}=-\delta_{\frac{1}{2}}, \beta_{r}=\delta_{r}-\delta_{r+\frac{1}{2}}\left(r \in \frac{1}{2} \mathbb{N}\right)\right\}, \\
\bar{\Delta}^{+} & =\left\{-\delta_{r}\left(r \in \frac{1}{2} \mathbb{N}\right),-2 \delta_{r}\left(r \in \frac{1}{2}+\mathbb{Z}_{+}\right), \pm \delta_{r}-\delta_{s}\left(r, s \in \frac{1}{2} \mathbb{N}, r<s\right)\right\} .
\end{aligned}
$$

- $\widehat{\mathrm{C}}$

$$
\begin{aligned}
\bar{\Pi}^{\vee} & =\left\{\beta_{0}^{\vee}=\widetilde{E}_{\frac{1}{2}}-\widetilde{E}_{1}+2 K, \beta_{r}^{\vee}=\widetilde{E}_{r}+\widetilde{E}_{r+\frac{1}{2}}\left(r \in \frac{1}{2} \mathbb{N}\right)\right\}, \\
\bar{\Pi} & =\left\{\beta_{0}=-\delta_{\frac{1}{2}}-\delta_{1}, \beta_{r}=\delta_{r}-\delta_{r+\frac{1}{2}}\left(r \in \frac{1}{2} \mathbb{N}\right)\right\}, \\
\bar{\Delta}^{+} & =\left\{-2 \delta_{n}(n \in \mathbb{N}), \pm \delta_{r}-\delta_{s}\left(r, s \in \frac{1}{2} \mathbb{N}, r<s\right)\right\} .
\end{aligned}
$$

- $\widehat{\mathcal{D}}$

$$
\begin{aligned}
\bar{\Pi}^{\vee} & =\left\{\beta_{0}^{\vee}=\widetilde{E}_{\frac{1}{2}}+K, \beta_{r}^{\vee}=\widetilde{E}_{r}+\widetilde{E}_{r+\frac{1}{2}}\left(r \in \frac{1}{2} \mathbb{N}\right)\right\}, \\
\bar{\Pi} & =\left\{\beta_{0}=-2 \delta_{\frac{1}{2}}, \beta_{r}=\delta_{r}-\delta_{r+\frac{1}{2}}\left(r \in \frac{1}{2} \mathbb{N}\right)\right\}, \\
\bar{\Delta}^{+} & =\left\{-2 \delta_{r}\left(r \in \frac{1}{2} \mathbb{N}\right), \pm \delta_{r}-\delta_{s}\left(r, s \in \frac{1}{2} \mathbb{N}, r<s\right)\right\} .
\end{aligned}
$$

\subsection{Tensor representations of $\mathfrak{g l}(m \mid n)$ and hook Schur functions.}


3.2.1. Hook Schur functions. Let $\lambda \in \mathcal{P}^{+}$and let $s_{\lambda}\left(x_{1}, y_{1}, x_{2}, y_{2}, \cdots\right)$ be the Schur function corresponding to $\lambda$. We can write (see e.g. [M])

$$
s_{\lambda}\left(x_{1}, y_{1}, x_{2}, y_{2}, x_{3}, y_{3}, \cdots\right)=\sum_{\mu \subseteq \lambda} s_{\mu}\left(x_{1}, x_{2}, \cdots\right) s_{\lambda / \mu}\left(y_{1}, y_{2}, \cdots\right) .
$$

Define the hook Schur function corresponding to $\lambda$ [BR, S1] to be

$$
H S_{\lambda}\left(x_{1}, y_{1}, x_{2}, y_{2}, \cdots\right)=\sum_{\mu \subseteq \lambda} s_{\mu}\left(x_{1}, x_{2}, \cdots\right) s_{(\lambda / \mu)^{\prime}}\left(y_{1}, y_{2}, \cdots\right) .
$$

From the definition it is not difficult to check the following (cf. $\mathrm{M}$, Chapter I $\S 5$ Ex. 23]).

Lemma 3.1. For a partition $\lambda$ we have

$$
H S_{\lambda^{\prime}}\left(x_{1}, y_{1}, x_{2}, y_{2}, \cdots\right)=H S_{\lambda}\left(y_{1}, x_{1}, y_{2}, x_{2}, \cdots\right) .
$$

3.2.2. Irreducible tensor representations of $\mathfrak{g l}(m \mid n)$. Below we present some basic results for the Lie superalgebra $\mathfrak{g l}(m \mid n)$, for $m \in \mathbb{N}$ and $n \in \mathbb{N} \cup\{\infty\}$, that will be used in the sequel.

Consider the space $\mathbb{C}^{m \mid n}$ spanned by basis elements $e_{i}$ with $i \in\{-m, \cdots,-1\} \cup$ $\{1,2, \cdots, n\}$, for $n$ finite, and $i \in\{-m, \cdots,-1\} \cup \mathbb{N}$, for $n=\infty$. We assume that $\operatorname{deg} e_{i}=\overline{0}$ for $i<0$, and $\operatorname{deg} e_{i}=\overline{1}$ for $i>0$. The Lie superalgebra $\mathfrak{g l}(m \mid n)$ is defined similarly as in Section 3.1.1. Denote the elementary matrices by $\left\{E_{i j}\right\}$. Here in Section 3.2 .2 our Borel subalgebra is $\sum_{i \leq j} \mathbb{C} E_{i j}$ with Cartan subalgebra $\overline{\mathfrak{h}}=\sum_{j} \mathbb{C} E_{j j}$. Let $\delta_{i} \in \overline{\mathfrak{h}}^{*}$ be defined by $\left\langle\delta_{i}, E_{j j}\right\rangle=\delta_{i j}$. We choose a symmetric bilinear form $(\cdot \mid \cdot)_{s}$ on $\overline{\mathfrak{h}}^{*}$ such that $\left(\delta_{i} \mid \delta_{j}\right)_{s}=-\operatorname{sgn}(i) \delta_{i j}$. For $\mu \in \overline{\mathfrak{h}}^{*}$ we denote by $L(\mathfrak{g l}(m \mid n), \mu)$ the irreducible highest weight module of highest weight $\mu$.

In this paragraph let us assume that $n$ is finite. It is well-known [BR, S1] that the tensor powers of the standard module $\mathbb{C}^{m \mid n}$ are completely reducible. The highest weights of the irreducible $\mathfrak{g l}(m \mid n)$-modules that appear as irreducible components in a tensor power of $\mathbb{C}^{m \mid n}$ are parameterized by the set

$$
\mathcal{P}_{m \mid n}^{+}:=\left\{\left(\lambda_{-m}, \lambda_{-m+1}, \cdots, \lambda_{-1}, \lambda_{1}, \lambda_{2}, \cdots\right) \in \mathcal{P}^{+} \mid \lambda_{1} \leq n\right\} .
$$

To be more precise, let us write $\lambda^{-}=\left(\lambda_{-m}, \cdots, \lambda_{-1}\right), \lambda^{+}=\left(\lambda_{1}, \lambda_{2}, \cdots\right)$ and $\lambda=$ $\left(\lambda^{-} \mid \lambda^{+}\right)$. The irreducible $\mathfrak{g l}(m \mid n)$-modules that appear in $\left(\mathbb{C}^{m \mid n}\right)^{\otimes k}$ have highest weights precisely of the form

$$
\lambda^{\natural}=\sum_{i=-m}^{-1} \lambda_{i} \delta_{i}+\sum_{j \geq 1} \lambda_{j}^{\prime} \delta_{j}, \quad|\lambda|=k .
$$

Such a module $L\left(\mathfrak{g l}(m \mid n), \lambda^{\natural}\right)$ will be called an irreducible tensor module. Furthermore, putting $e^{\delta_{i}}=x_{i}(i<0)$ and $y_{j}=e^{\delta_{j}}(j>0)$ the character of $L\left(\mathfrak{g l}(m \mid n), \lambda^{\natural}\right)$ 
is given by [BR, $\mathrm{S} 1]$

$$
\sum_{\mu \subseteq \lambda} s_{\mu}\left(x_{-m}, \cdots, x_{-1}\right) s_{(\lambda / \mu)^{\prime}}\left(y_{1}, y_{2}, \cdots\right) .
$$

For $n \in \mathbb{N} \cup\{\infty\}$ let $X^{m \mid n}$ denote the set of sequences of $m+n$ integers, which we index by the set $\{-m, \cdots,-1,1, \cdots, n\}$ for $n \in \mathbb{N}$, and $\{-m, \cdots,-1\} \cup \mathbb{N}$ for $n=\infty$. We assume that a sequence in $X^{m \mid n}$ has only finitely many non-zero entries. Let $X_{+}^{m \mid n} \subseteq X^{m \mid n}$ be the subset consisting of sequences of the form $\mu=\left(\mu^{-} \mid \mu^{+}\right)$, where $\mu^{-}=\left(\mu_{-m}, \cdots, \mu_{-1}\right)$ is a generalized partition of length $m$ and $\mu^{+}=\left(\mu_{1}, \mu_{2}, \ldots\right)$ is a partition. We may regard $\mu \in X^{m \mid n}$ as the element $\sum_{i \geq-m, i \neq 0} \mu_{i} \delta_{i} \in \overline{\mathfrak{h}}^{*}$.

Consider the category $\mathcal{O}_{m \mid n}^{+}$of $\overline{\mathfrak{h}}$-semisimple $\mathfrak{g l}(m \mid n)$-modules consisting of objects $M$ that have composition series of the form

$$
0 \subseteq M_{1} \subseteq M_{2} \subseteq M_{3} \subseteq \cdots
$$

with $\bigcup_{i=1}^{\infty} M_{i}=M$, and such that each composition factor is of the form $L(\mathfrak{g l}(m \mid n), \mu)$, $\mu \in X_{+}^{m \mid n}$. Furthermore we assume that each isotypic composition factor appears with finite multiplicity.

Recall the truncation functor of [CWZ, Definition 4.4] defined as follows. Let $n$ be finite and let $\mu \in X_{+}^{m \mid \infty}$. Note that in the case $\left(\mu \mid \delta_{n+1}\right)_{s}=0$ we may regard $\mu$ as a weight of $\mathfrak{g l}(m \mid n)$ by ignoring the zeros. Define for $M \in \mathcal{O}_{m \mid \infty}^{+}$a functor $\mathfrak{t r}_{n}: \mathcal{O}_{m \mid \infty}^{+} \rightarrow \mathcal{O}_{m \mid n}^{+}$by setting $\mathfrak{t r}_{n}(M)$ to be the linear span of all weight vectors $m \in M$ such that $\left(\operatorname{wt}(m) \mid \delta_{j}\right)_{s}=0$ for $j>n$, where wt $(m)$ denotes the weight of $m$. Clearly $\mathfrak{t r}_{n}$ is an exact functor. It is shown in [CWZ, Corollary 3.6] that

$$
\mathfrak{t r}_{n}(L(\mathfrak{g l}(m \mid \infty), \mu))= \begin{cases}L(\mathfrak{g l}(m \mid n), \mu), & \text { if }\left(\mu \mid \delta_{n+1}\right)_{s}=0 \\ 0, & \text { otherwise. }\end{cases}
$$

Lemma 3.2. The $\mathfrak{g l}(m \mid \infty)$-module $\left(\mathbb{C}^{m \mid \infty}\right)^{\otimes k}$ is completely reducible. Furthermore we have

$$
\left(\mathbb{C}^{m \mid \infty}\right)^{\otimes k} \cong \bigoplus_{\lambda} L\left(\mathfrak{g l}(m \mid \infty), \lambda^{\natural}\right)^{m_{\lambda}}
$$

where the sum ranges over all partitions $\lambda=\left(\lambda_{-m}, \cdots, \lambda_{-1}, \lambda_{1}, \lambda_{2}, \cdots\right)$ with $|\lambda|=k$, and $m_{\lambda} \in \mathbb{N}$.

Proof. Suppose that in $\left(\mathbb{C}^{m \mid \infty}\right)^{\otimes k}$ we have a non-trivial extension of the form

$$
0 \longrightarrow L(\mathfrak{g l}(m \mid \infty), \gamma) \longrightarrow E \longrightarrow L(\mathfrak{g l}(m \mid \infty), \mu) \longrightarrow 0 .
$$

Take a finite $n \gg 0$ such that $\left(\gamma \mid \delta_{j}\right)_{s}=\left(\mu \mid \delta_{j}\right)_{s}=0$, for $j \geq n+1$. Applying the truncation functor $\mathfrak{t r}_{n}$ upon (3.5) we get an exact sequence of $\mathfrak{g l}(m \mid n)$-modules

$$
0 \longrightarrow L(\mathfrak{g l}(m \mid n), \gamma) \longrightarrow \mathfrak{t r}_{n} E \longrightarrow L(\mathfrak{g l}(m \mid n), \mu) \longrightarrow 0 .
$$


Since $\mathfrak{t r}_{n}\left(\mathbb{C}^{m \mid \infty}\right)^{\otimes k}=\left(\mathbb{C}^{m \mid n}\right)^{\otimes k}, \mathfrak{t r}_{n} E$ is an extension inside $\left(\mathbb{C}^{m \mid n}\right)^{\otimes k}$, which therefore must be a split extension. Thus we may assume that there exists $v_{\mu} \in E$ that is annihilated by all $E_{i, i+1}$, for $i \leq n-1$. Now $E_{i, i+1} v_{\mu}$ has weight $\mu+\delta_{i}-\delta_{i+1}$, for $i>n$. But there are no such weight spaces in $E$, being an extension of two highest weight modules of highest weights $\gamma$ and $\mu$. Thus $E_{i, i+1} v_{\mu}=0$, for $i \geq n$, and hence $v_{\mu}$ is a genuine highest weight vector inside $E$. Now $U(\mathfrak{g l}(m \mid k)) v_{\mu}=$ $L(\mathfrak{g l}(m \mid k), \mu)$, for all $k \geq n$, and hence $U(\mathfrak{g l}(m \mid \infty)) v_{\mu}=L(\mathfrak{g l}(m \mid \infty), \mu)$. It follows that $U(\mathfrak{g l}(m \mid \infty)) v_{\mu} \cap U(\mathfrak{g l}(m \mid \infty)) v_{\gamma}=0$, and thus (3.5) is a split extension.

The second statement is easy using the truncation functor again together with the statement for finite $n$.

Lemma 3.2 allows us to define irreducible tensor representations of $\mathfrak{g l}(m \mid \infty)$ in an analogous fashion. It follows that they are parameterized by $\mathcal{P}^{+}$. Furthermore the character of $L\left(\mathfrak{g l}(m \mid \infty), \lambda^{\natural}\right)$ is given by (3.3) with $n=\infty$.

Assume that $n$ is finite. Let $\gamma \in X^{m \mid n}$. We denote the central character corresponding to $\gamma$ by $\chi_{\gamma}$ (see e.g. [Se, $[\mathrm{B}]$ ). Weights in $\overline{\mathfrak{h}}^{*}$ corresponding to the same central character can be characterized as follows. Let $P$ be the free abelian group with basis $\left\{\varepsilon_{a} \mid a \in \mathbb{Z}\right\}$. Define

$$
\varepsilon_{\gamma}:=\sum_{i=-m}^{-1} \varepsilon_{\gamma_{i}-i}-\sum_{j=1}^{n} \varepsilon_{-\gamma_{j}+j} .
$$

Then $\chi_{\gamma}=\chi_{\mu}$ if and only if $\varepsilon_{\gamma}=\varepsilon_{\mu}$ (see [B, Lemma 4.18]). Returning to tensor modules, take a partition $\lambda=\left(\lambda_{-m}, \cdots, \lambda_{-1}, \lambda_{1}, \lambda_{2}, \cdots\right)$ with $\lambda_{1} \leq n$. Consider

$$
\varepsilon_{\lambda^{\natural}}=\sum_{i=-m}^{-1} \varepsilon_{\lambda_{i}-i}-\sum_{j=1}^{n} \varepsilon_{-\lambda_{j}^{\prime}+j} .
$$

Lemma 3.3. Let $\lambda \in \mathcal{P}_{m \mid n}^{+}$and suppose that for some $i, j>0$ we have $\lambda_{-j}+j=$ $-\lambda_{i}^{\prime}+i$. Then we have $\lambda_{-j}=i-j, \lambda_{i}^{\prime}=0$, and hence $\lambda_{s}^{\prime}=0$, for $s=i+1, \cdots, n$.

Proof. The equality $\lambda_{-j}+j=-\lambda_{i}^{\prime}+i$ implies that $\lambda_{-j}+\lambda_{i}^{\prime}=i-j$. Now if $\lambda_{i}^{\prime}>0$, then $\lambda_{-j} \geq i$. But then the identity implies that $\lambda_{i}^{\prime}=0$.

Lemma 3.4. The map given by $\lambda \mapsto \varepsilon_{\lambda^{\natural}}$ is an injection from $\mathcal{P}_{m \mid n}^{+}$to $P$.

Proof. We will show that we can construct the partition $\lambda$ from $\varepsilon_{\lambda^{\natural}}$.

In light of the Lemma 3.3 we can construct $\lambda$ as follows. Consider

$$
\varepsilon_{\lambda^{\natural}}=\varepsilon_{a_{1}}+\cdots+\varepsilon_{a_{s}}-\varepsilon_{b_{1}}-\cdots-\varepsilon_{b_{t}},
$$

with $a_{1}>a_{2}>\cdots>a_{s}$, and $b_{1}<b_{2}<\cdots<b_{t}$. The total number of terms is $m+n-2 k$, where $k$ is the degree of atypicality of $\lambda$. Let

$$
\varepsilon_{-}^{0}=-\varepsilon_{b_{1}}-\cdots-\varepsilon_{b_{t}} .
$$


Assume that $k>0$. Otherwise it is clear what $\lambda$ is. Let $c_{0} \leq n$ be the smallest positive integer such that $-\varepsilon_{c_{0}+1}-\varepsilon_{c_{0}+2}-\cdots-\varepsilon_{n}$ is a summand of (3.7) above. Then we define

$$
\varepsilon_{-}^{1}=-\varepsilon_{b_{1}}-\cdots-\varepsilon_{b_{t}}-\varepsilon_{c_{0}} .
$$

Now we let $c_{1}<c_{0}$ be the smallest integer such that $-\varepsilon_{c_{1}+1}-\cdots-\varepsilon_{n}$ is a summand of (3.8). Define $\varepsilon_{-}^{2}$ analogously et cetera until we get

$$
\varepsilon_{-}^{k}=\varepsilon_{-}^{0}-\sum_{i=0}^{k-1} \varepsilon_{c_{i}}
$$

Consider

$$
\varepsilon_{\lambda^{\natural}}=\left(\varepsilon_{a_{1}}+\cdots+\varepsilon_{a_{s}}+\sum_{i=0}^{k-1} \varepsilon_{c_{i}}\right)-\left(\varepsilon_{b_{1}}+\cdots+\varepsilon_{b_{t}}+\sum_{i=0}^{k-1} \varepsilon_{c_{i}}\right) .
$$

From this form with $m+n$ summands we can read off $\lambda$ easily.

Corollary 3.1. Let $n \in \mathbb{N}$. The irreducible tensor representations have distinct central characters. In particular for $\lambda, \mu \in \mathcal{P}_{m \mid n}^{+}$we have

$$
\operatorname{Ext}^{1}\left(L\left(\mathfrak{g l}(m \mid n), \lambda^{\natural}\right), L\left(\mathfrak{g l}(m \mid n), \mu^{\natural}\right)\right)=0 .
$$

Let $\mathcal{O}_{m \mid n}^{++}$denote the full subcategory of $\mathcal{O}_{m \mid n}^{+}$such that each composition factor isomorphic to an irreducible tensor module.

Remark 3.1. Let $n \in \mathbb{N}$ and let $\mathcal{O}_{m \mid n}^{++, f}$ denote the full subcategory of $\mathcal{O}_{m \mid n}^{++}$consisting of objects that have finite composition series. Then $\mathcal{O}_{m \mid n}^{++, f}$ is a full subcategory of the category of finite-dimensional $\mathfrak{g l}(m \mid n)$-modules. From Corollary 3.1 and the long exact sequence one shows, using induction on the length of the composition series, that $\operatorname{Ext}^{1}(M, N)=0$, for $M, N \in \mathcal{O}_{m \mid n}^{++, f}$. The projective cover $P\left(\mathfrak{g l}(m \mid n), \lambda^{\natural}\right)$ of $L\left(\mathfrak{g l}(m \mid n), \lambda^{\natural}\right)$ exists in the category of finite-dimensional $\mathfrak{g l}(m \mid n)$-modules (see e.g. [B]). Now consider the short exact sequence

$$
0 \longrightarrow K \longrightarrow P\left(\mathfrak{g l}(m \mid n), \lambda^{\natural}\right) \longrightarrow L\left(\mathfrak{g l}(m \mid n), \lambda^{\natural}\right) \longrightarrow 0 .
$$

From the long exact sequence one shows that $\operatorname{Ext}^{i}(M, N)=0$, and in particular, $\operatorname{Ext}^{i}\left(L\left(\mathfrak{g l}(m \mid n), \lambda^{\natural}\right), L\left(\mathfrak{g l}(m \mid n), \mu^{\natural}\right)\right)=0$, for all $i \in \mathbb{N}$.

Theorem 3.1. For $n \in \mathbb{N} \cup\{\infty\}$ the category $\mathcal{O}_{m \mid n}^{++}$is a semisimple tensor category.

Proof. Given $M, N \in \mathcal{O}_{m \mid n}^{++}$with composition series $0 \subseteq M_{1} \subseteq M_{2} \subseteq \ldots$, and $0 \subseteq N_{1} \subseteq N_{2} \subseteq \ldots$, respectively. We obtain a filtration of $M \otimes N$

$$
0 \subseteq M_{1} \otimes N_{1} \subseteq M_{2} \otimes N_{2} \subseteq \ldots,
$$


whose composition series is a composition series for $M \otimes N$. Now for $\lambda, \mu, \nu \in \mathcal{P}_{m \mid n}^{+}$, $L\left(\lambda^{\natural}\right) \subseteq L\left(\mu^{\natural}\right) \otimes L\left(\nu^{\natural}\right)$ only if $|\lambda|=|\mu|+|\nu|$. Thus every isotypic composition factor of $M \otimes N$ appears with finite multiplicity. Hence $\mathcal{O}_{m \mid n}^{++}$is a tensor category.

The theorem for a finite $n$ now follows from Corollary 3.1. To show the statement for $n=\infty$ we need to show that any exact sequence of $\mathfrak{g l}(m \mid \infty)$-modules of the form

$$
0 \longrightarrow L\left(\mathfrak{g l}(m \mid \infty), \lambda^{\natural}\right) \longrightarrow E \longrightarrow L\left(\mathfrak{g l}(m \mid \infty), \mu^{\natural}\right) \longrightarrow 0
$$

is split. For this choose a finite $n$ sufficiently large so that $\lambda_{1}, \mu_{1} \leq n$. Applying the truncation functor $\mathfrak{t r}_{n}$ we get an exact sequence of $\mathfrak{g l}(m \mid n)$-modules

$$
0 \longrightarrow L\left(\mathfrak{g l}(m \mid n), \lambda^{\natural}\right) \longrightarrow \mathfrak{t r}_{n} E \longrightarrow L\left(\mathfrak{g l}(m \mid n), \mu^{\natural}\right) \longrightarrow 0,
$$

which is split. Now repeating the arguments of Lemma 3.2 completes the proof.

3.2.3. Non-standard Borel subalgebra of $\mathfrak{g l}(m \mid m)$. Below we will discuss the highest weight theory of the Lie superalgebra $\mathfrak{g l}(m \mid m)$, for $m \in \mathbb{N} \cup\{\infty\}$, with respect to the non-standard Borel subalgebra.

Let $\mathbb{C}^{m \mid m}=\mathbb{C}^{>0}$ be the superspace of dimension $(m \mid m)$. We assume that it has a basis $\left\{e_{r} \mid r=\frac{1}{2}, 1, \frac{3}{2}, 2, \cdots\right\}$ with $\operatorname{deg} e_{r}=\overline{0}$ for $r \in\{1,2, \cdots\}$, and $\operatorname{deg} e_{s}=\overline{1}$ otherwise. With respect to this basis the subalgebra generated by $E_{r s}(r \leq s)$ will be called the non-standard Borel subalgebra of $\mathfrak{g l}(m \mid m)$.

For a finite $m$ it is clear that $\left(\mathbb{C}^{m \mid m}\right)^{\otimes k}$ is a completely reducible $\mathfrak{g l}(m \mid m)$-module, and the irreducible factors are the irreducible tensor representations, which are parameterized by $\lambda=\left(\lambda_{1}, \lambda_{2}, \ldots\right) \in \mathcal{P}^{+}$with $\lambda_{m+1} \leq m$. In fact, for such a $\lambda$, the highest weight (with respect to the non-standard Borel subalgebra and the Cartan subalgebra $\left.\sum_{r} \mathbb{C} E_{r r}\right)$ of the corresponding representation of $\mathfrak{g l}(m \mid m)$ is given by $\sum_{r} a_{r} \delta_{r}$ with (cf. e.g. [CW2, Section 7])

$$
a_{r}= \begin{cases}\left\langle\lambda_{r+\frac{1}{2}}^{\prime}-\left(r-\frac{1}{2}\right)\right\rangle, & \text { if } r \in\left\{\frac{1}{2}, \frac{3}{2}, \cdots\right\}, \\ \left\langle\lambda_{r}-r\right\rangle, & \text { if } r \in\{1,2, \cdots\} .\end{cases}
$$

Above $\langle k\rangle=k$, for $k>0$, and $\langle k\rangle=0$, otherwise. By (3.3) its character, which is the trace of the operator $\prod_{r} x_{r}^{E_{r r}}$, is $H S_{\lambda^{\prime}}\left(x_{\frac{1}{2}}, x_{1}, x_{\frac{3}{2}}, \cdots\right)$, since the standard and non-standard Borel subalgebras share the same Cartan subalgebra.

For $m=\infty$ it is not hard to show, with the help of a similarly defined truncation functor, that an analogous statement of Lemma 3.2 remains valid in the setup of non-standard Borel.

We define respective categories $\mathcal{O}_{m \mid m}^{++}(m \in \mathbb{N} \cup\{\infty\})$ which is a semisimple tensor category for $m \in \mathbb{N}$ by Theorem 3.1. Using this fact we then can show in an analogous fashion the following.

Theorem 3.2. The category $\mathcal{O}_{\infty \mid \infty}^{++}$is a semisimple tensor category. 
To simplify notation for later on we set, for $m=\infty$ above, $\overline{\mathfrak{g l}}_{>0}:=\mathfrak{g l}(\infty \mid \infty)$, which may be regarded as a subalgebra of $\widehat{\mathfrak{g l}}_{\infty \mid \infty}$ spanned by $E_{r s}$ with $r, s>0$. Let $\overline{\mathfrak{g l}}_{\leq 0}$ be the subalgebra of $\widehat{\mathfrak{g l}}_{\infty \mid \infty}$ spanned by $E_{r s}$ with $r, s \leq 0$, which is isomorphic to $\overline{\mathfrak{g l}}_{>0}$.

Let $\mathbb{C} \leq 0=\sum_{r \in-\frac{1}{2} \mathbb{Z}_{+}} \mathbb{C} e_{r}$. Similarly, we call an irreducible component of $(\mathbb{C} \leq 0 *)^{\otimes k}$ an irreducible dual tensor representation of $\overline{\mathfrak{g l}}_{\leq 0}$. As in $\left(\mathbb{C}^{>0}\right)^{\otimes k}$, each irreducible component corresponds to $\lambda \in \mathcal{P}^{+}$, whose highest weight with respect to the nonstandard Borel subalgebra $\sum_{r \leq s} \mathbb{C} E_{r s}$ and the Cartan subalgebra $\sum_{r \in-\frac{1}{2} \mathbb{Z}_{+}} \mathbb{C} E_{r r}$ is given by

$$
-\sum_{r \in-\frac{1}{2} \mathbb{Z}_{+}} b_{r} \delta_{r}, \quad \text { where } b_{r}= \begin{cases}\left\langle\lambda_{-r+1}+r\right\rangle, & \text { if } r \in-\mathbb{Z}_{+}, \\ \left\langle\lambda_{-r+\frac{1}{2}}^{\prime}+\left(r-\frac{1}{2}\right)\right\rangle, & \text { if } r \in-\frac{1}{2}-\mathbb{Z}_{+} .\end{cases}
$$

Its character is $H S_{\lambda}\left(x_{0}^{-1}, x_{-\frac{1}{2}}^{-1}, x_{-1}^{-1}, \ldots\right)$ and we have an analogous statement on semisimplicity of an analogous category of dual tensor representations.

3.3. Super dual pairs on infinite-dimensional Fock spaces. Let $\overline{\mathfrak{g}}$ be one of the Lie superalgebras defined in Section 3.1. Let $\Lambda \in \overline{\mathfrak{h}}^{*}$ be given. By standard arguments there exists a unique irreducible highest weight $\overline{\mathfrak{g}}$-module of highest weight $\Lambda$, which will be denoted by $L(\overline{\mathfrak{g}}, \Lambda)$. For $\Lambda \in \overline{\mathfrak{h}}^{*}$, we set $\Lambda_{s}=\left\langle\Lambda, E_{s s}\right\rangle\left(s \in \frac{1}{2} \mathbb{Z}\right)$ if $\overline{\mathfrak{g}}=\widehat{\mathfrak{g l}}_{\infty \mid \infty}$, and $\Lambda_{s}=\left\langle\Lambda, \widetilde{E}_{s}\right\rangle\left(s \in \frac{1}{2} \mathbb{N}\right)$ if $\overline{\mathfrak{g}}=\widehat{\mathcal{B}}, \widehat{\mathrm{C}}, \widehat{\mathcal{D}}$.

Fix a positive integer $\ell \geq 1$. Consider $\ell$ pairs of free fermions $\psi^{ \pm, i}(z)$ and $\ell$ pairs of free bosons $\gamma^{ \pm, i}(z)$ with $i=1, \cdots, \ell$. That is, we have

$$
\begin{array}{rlrl}
\psi^{+, i}(z) & =\sum_{n \in \mathbb{Z}} \psi_{n}^{+, i} z^{-n-1}, & \psi^{-, i}(z) & =\sum_{n \in \mathbb{Z}} \psi_{n}^{-, i} z^{-n}, \\
\gamma^{+, i}(z) & =\sum_{r \in \frac{1}{2}+\mathbb{Z}} \gamma_{r}^{+, i} z^{-r-1 / 2}, & \gamma^{-, i}(z)=\sum_{r \in \frac{1}{2}+\mathbb{Z}} \gamma_{r}^{-, i} z^{-r-1 / 2},
\end{array}
$$

with non-trivial commutation relations

$$
\left[\psi_{m}^{+, i}, \psi_{n}^{-, j}\right]=\delta_{i j} \delta_{m+n, 0}, \quad\left[\gamma_{r}^{+, i}, \gamma_{s}^{-, j}\right]=\delta_{i j} \delta_{r+s, 0} .
$$

Let $\overline{\mathfrak{F}}^{\ell}$ denote the corresponding Fock space generated by the vaccum vector $|0\rangle$, which is annihilated by $\psi_{n}^{+, i}, \psi_{m}^{-, i}, \gamma_{r}^{ \pm, i}$ for $n \geq 0, m>0$ and $r>0$.

We also consider the following $\ell$ pairs of free fermions $\widetilde{\psi}^{ \pm, i}(i=1, \cdots, \ell)$ :

$$
\widetilde{\psi}^{+, i}(z)=\sum_{n \in \mathbb{Z} \backslash\{0\}} \psi_{n}^{+, i} z^{-n-1}, \quad \widetilde{\psi}^{-, i}(z)=\sum_{n \in \mathbb{Z} \backslash\{0\}} \psi_{n}^{-, i} z^{-n},
$$

with the same commutation relations. We denote by $\overline{\mathfrak{F}}_{0}^{\ell}$ the Fock space corresponding to $\widetilde{\psi}^{ \pm, i}(z)$ and $\gamma^{ \pm, i}(z)(i=1, \cdots, \ell)$ generated by the vacuum vector $|0\rangle$ with $\psi_{n}^{ \pm, i}|0\rangle=\gamma_{r}^{ \pm, i}|0\rangle=0$, for $n, r>0$. 
We introduce a neutral fermionic field $\widetilde{\phi}(z)=\sum_{n \in \mathbb{Z} \backslash\{0\}} \phi_{n} z^{-n-1}$ and a neutral bosonic field $\chi(z)=\sum_{r \in \frac{1}{2}+\mathbb{Z}} \chi_{r} z^{-r-\frac{1}{2}}$ with non-trivial commutation relations $\left[\phi_{m}, \phi_{n}\right]=\delta_{m+n, 0}$ and $\left[\chi_{r}, \chi_{s}\right]=\delta_{r+s, 0}$. Denote by $\overline{\mathfrak{F}}_{0}^{\frac{1}{2}}$ the corresponding Fock space generated by the vacuum vector $|0\rangle$, which is annihilated by $\phi_{m}$ and $\chi_{r}$ for $m, r>0$. We denote by $\overline{\mathfrak{F}}_{0}^{\ell+\frac{1}{2}}$ the tensor product of $\overline{\mathfrak{F}}_{0}^{\ell}$ and $\overline{\mathfrak{F}}_{0}^{\frac{1}{2}}$.

Let $W$ be the Weyl group of the Lie algebra $\mathfrak{g}$, and $G$ the dual partner of $\mathfrak{g}$ in Section 2.3. We let $W_{k}^{0}, \lambda_{w}^{ \pm}, \lambda_{w}\left(\lambda \in \mathcal{P}(G), w \in W_{k}^{0}\right)$ be as in Section 2.4, Let $x_{r}$ $\left(r \in \frac{1}{2} \mathbb{Z}\right)$ and $z_{i}(i=1, \cdots, \ell)$ be formal indeterminates.

3.3.1. The $\left(\widehat{\mathfrak{g l}}_{\infty \mid \infty}, \mathrm{GL}(\ell)\right)$-duality. Given $\lambda \in \mathcal{P}(\operatorname{GL}(\ell))$, we define $\bar{\Lambda}^{\mathfrak{a}}(\lambda) \in \overline{\mathfrak{h}}^{*}$ by

$$
\begin{aligned}
& \bar{\Lambda}^{\mathfrak{a}}(\lambda)_{i}=\left\langle\lambda_{i}^{\prime}-i\right\rangle, \quad i \in \mathbb{N}, \\
& \bar{\Lambda}^{\mathfrak{a}}(\lambda)_{j}=-\left\langle-\lambda_{j}^{\prime}+j\right\rangle, \quad j \in-\mathbb{Z}_{+}, \\
& \bar{\Lambda}^{\mathfrak{a}}(\lambda)_{r}=\left\langle\lambda_{r+1 / 2}-(r-1 / 2)\right\rangle, \quad r \in \frac{1}{2}+\mathbb{Z}_{+}, \\
& \bar{\Lambda}^{\mathfrak{a}}(\lambda)_{s}=-\left\langle-\lambda_{\ell+(s+1 / 2)}+(s-1 / 2)\right\rangle, \quad s \in-\frac{1}{2}-\mathbb{Z}_{+}, \\
& \left\langle\bar{\Lambda}^{\mathfrak{a}}(\lambda), K\right\rangle=\ell .
\end{aligned}
$$

Proposition 3.1. CW2, Theorem 8.1] There exists an action of $\widehat{\mathfrak{g l}}_{\infty \mid \infty} \times \mathrm{GL}(\ell)$ on $\overline{\mathfrak{F}}$. Furthermore, under this joint action, we have

$$
\overline{\mathfrak{F}}^{\ell} \cong \bigoplus_{\lambda \in \mathcal{P}(\operatorname{GL}(\ell))} L\left(\widehat{\mathfrak{g l}}_{\infty \mid \infty}, \bar{\Lambda}^{\mathfrak{a}}(\lambda)\right) \otimes V_{\mathrm{GL}(\ell)}^{\lambda}
$$

Computing the trace of the operator $\prod_{s \in \frac{1}{2} \mathbb{Z}} x_{s}^{E_{s s}} \prod_{i=1}^{\ell} z_{i}^{e_{i i}}$ on both sides of (3.9), we obtain the following identity:

$$
\prod_{i=1}^{\ell} \frac{\prod_{n \in \mathbb{N}}\left(1+x_{n} z_{i}\right)\left(1+x_{-n+1}^{-1} z_{i}^{-1}\right)}{\prod_{r \in 1 / 2+\mathbb{Z}_{+}}\left(1-x_{r} z_{i}\right)\left(1-x_{-r}^{-1} z_{i}^{-1}\right)}=\sum_{\lambda \in \mathcal{P}(\mathrm{GL}(\ell))} \operatorname{ch} L\left(\widehat{\mathfrak{g l}}_{\infty \mid \infty}, \bar{\Lambda}^{\mathfrak{a}}(\lambda)\right) \operatorname{ch} V_{\mathrm{GL}(\ell)}^{\lambda} .
$$

Set $\mathbf{x}_{>0}=\left\{x_{\frac{1}{2}}, x_{1}, x_{\frac{3}{2}}, \cdots\right\}, \mathbf{x}_{\leq 0}^{-1}=\left\{x_{0}^{-1}, x_{-\frac{1}{2}}^{-1}, x_{-1}^{-1}, \cdots\right\}$, and

$$
\bar{D}^{\mathfrak{a}}=\prod_{i, j \in \mathbb{N}} \frac{\left(1-x_{-i+1}^{-1} x_{j}\right)\left(1-x_{-i+\frac{1}{2}}^{-1} x_{j-\frac{1}{2}}\right)}{\left(1+x_{-i+1}^{-1} x_{j-\frac{1}{2}}\right)\left(1+x_{-i+\frac{1}{2}}^{-1} x_{j}\right)}
$$


Theorem 3.3. For $\lambda \in \mathcal{P}(\mathrm{GL}(\ell))$, we have

$$
\operatorname{ch} L\left(\widehat{\mathfrak{g l}}_{\infty \mid \infty}, \bar{\Lambda}^{\mathfrak{a}}(\lambda)\right)=\frac{1}{\bar{D}^{\mathfrak{a}}} \sum_{k=0}^{\infty} \sum_{w \in W_{k}^{0}}(-1)^{k} H S_{\left(\lambda_{w}^{+}\right)^{\prime}}\left(\mathbf{x}_{>0}\right) H S_{\lambda_{w}^{-}}\left(\mathbf{x}_{\leq 0}^{-1}\right) .
$$

Proof. The proof is similar to that of Theorem 3.2 in [CL]. First we use (2.17) and (2.18) to write (2.5) as an identity in $\left\{x_{-j}^{-1}\right\}_{j \in \mathbb{Z}_{+}}$and $\left\{x_{j}\right\}_{j \in \mathbb{N}}$. Next we replace $\left\{x_{-j}^{-1}\right\}_{j \in \mathbb{Z}_{+}}$(resp. $\left\{x_{j}\right\}_{j \in \mathbb{N}}$ ) with $\left\{x_{-j}^{-1}\right\}_{j \in \frac{1}{2} \mathbb{Z}_{+}}$(resp. $\left\{x_{j}\right\}_{j \in \frac{1}{2} \mathbb{N}}$ ) in this new identity, which we regard first as a symmetric function identity in the variables $\left\{x_{-j}^{-1}\right\}_{j \in \frac{1}{2}+\mathbb{Z}_{+}}$. This allows us to apply to it the involution of symmetric functions that interchanges the elementary symmetric functions with the complete symmetric functions (see e.g. [M, Chapter I, $\S 2,(2.7)]$ ) and obtain, using Lemma 3.1.

$$
\begin{aligned}
& \prod_{k=1}^{\ell} \prod_{i \in \mathbb{N}} \prod_{j \in \frac{1}{2} \mathbb{N}} \frac{\left(1+x_{j} z_{k}\right)\left(1+x_{-i+1}^{-1} z_{k}^{-1}\right)}{\left(1-x_{-i+\frac{1}{2}}^{-1} z_{k}^{-1}\right)}=\sum_{\lambda \in \mathcal{P}(\mathrm{GL}(\ell))} \operatorname{ch} V_{\mathrm{GL}(\ell)}^{\lambda} \times \\
& \frac{\sum_{k=0}^{\infty} \sum_{w \in W_{k}^{0}}(-1)^{k} s_{\lambda_{w}^{+}}\left(x_{\frac{1}{2}}, x_{1}, \cdots\right) H S_{\lambda_{w}^{-}}\left(x_{0}^{-1}, x_{-\frac{1}{2}}^{-1}, x_{-1}^{-1}, \cdots\right)}{\prod_{i \in \mathbb{N}, j \in \frac{1}{2} \mathbb{N}}\left(1-x_{-i+1}^{-1} x_{j}\right) /\left(1+x_{-i+\frac{1}{2}}^{-1} x_{j}\right)} .
\end{aligned}
$$

Now we regard (3.11) as a symmetric function identity in the variables $\left\{x_{j}\right\}_{j \in \frac{1}{2}+\mathbb{Z}_{+}}$ and apply the involution again to obtain, using Lemma 3.1.

$$
\begin{aligned}
\prod_{k=1}^{\ell} & \frac{\prod_{n \in \mathbb{N}}\left(1+x_{n} z_{k}\right)\left(1+x_{-n+1}^{-1} z_{k}^{-1}\right)}{\prod_{r \in 1 / 2+\mathbb{Z}_{+}}\left(1-x_{r} z_{k}\right)\left(1-x_{-r}^{-1} z_{k}^{-1}\right)}=\sum_{\lambda \in \mathcal{P}(\mathrm{GL}(\ell))} \operatorname{ch} V_{\mathrm{GL}(\ell)}^{\lambda} \times \\
& \frac{\sum_{k=0}^{\infty} \sum_{w \in W_{k}^{0}}(-1)^{k} H S_{\left(\lambda_{w}^{+}\right)^{\prime}}\left(x_{\frac{1}{2}}, x_{1}, \cdots\right) H S_{\lambda_{w}^{-}}\left(x_{0}^{-1}, x_{-\frac{1}{2}}^{-1}, x_{-1}^{-1}, \cdots\right)}{\prod_{i, j \in \mathbb{N}}\left(1-x_{-i+1}^{-1} x_{j}\right)\left(1-x_{-i+\frac{1}{2}}^{-1} x_{j-\frac{1}{2}}\right) /\left(1+x_{-i+1}^{-1} x_{j-\frac{1}{2}}\right)\left(1+x_{-i+\frac{1}{2}}^{-1} x_{j}\right)} .
\end{aligned}
$$

Now the theorem follows from (3.10) and the fact that the set $\left\{\operatorname{ch} V_{\mathrm{GL}(\ell)}^{\lambda} \mid \lambda \in \mathcal{P}(\mathrm{GL}(\ell))\right\}$, being the set of Schur Laurent polynomials, is linearly independent.

We record an identity (cf. [CW1, Remark 3.3]) that can be proved similarly as Theorem 3.3 using the classical Cauchy identity:

$$
\prod_{i=1}^{\ell} \frac{\prod_{n \in \mathbb{N}}\left(1+x_{n} z_{i}\right)}{\prod_{r \in 1 / 2+\mathbb{Z}_{+}}\left(1-x_{r} z_{i}\right)}=\sum_{\lambda \in \mathcal{P}^{+}, l(\lambda) \leq \ell} H S_{\lambda^{\prime}}\left(\mathbf{x}_{>0}\right) s_{\lambda}\left(z_{1}, \cdots, z_{\ell}\right) .
$$

3.3.2. The $(\widehat{\mathcal{B}}, \operatorname{Pin}(2 \ell))$-duality. For $\lambda \in \mathcal{P}(\operatorname{Pin}(2 \ell))$, we define $\bar{\Lambda}^{\mathfrak{b}}(\lambda) \in \overline{\mathfrak{h}}^{*}$ by

$$
\begin{aligned}
& \bar{\Lambda}^{\mathfrak{b}}(\lambda)_{i}=\left\langle\lambda_{i}^{\prime}-i\right\rangle, \quad i \in \mathbb{N}, \\
& \bar{\Lambda}^{\mathfrak{b}}(\lambda)_{r}=\left\langle\lambda_{r+1 / 2}-(r-1 / 2)\right\rangle, \quad r \in \frac{1}{2}+\mathbb{Z}_{+}, \\
& \left\langle\bar{\Lambda}^{\mathfrak{b}}(\lambda), K\right\rangle=\ell .
\end{aligned}
$$


Proposition 3.2. CW2, Theorem 8.2] There exists an action of $\widehat{\mathcal{B}} \times \operatorname{Pin}(2 \ell)$ on $\overline{\mathfrak{F}}^{\ell}$. Furthermore, under this joint action, we have

$$
\overline{\mathfrak{F}}^{\ell} \cong \bigoplus_{\lambda \in \mathcal{P}(\operatorname{Pin}(2 \ell))} L\left(\widehat{\mathcal{B}}, \bar{\Lambda}^{\mathfrak{b}}(\lambda)\right) \otimes V_{\operatorname{Pin}(2 \ell)}^{\lambda}
$$

Computing the trace of the operator $\prod_{s \in \frac{1}{2} \mathbb{N}} x_{s}^{\widetilde{E}_{s}} \prod_{i=1}^{\ell} z_{i}^{\tilde{e}_{i}}$ on both sides of (3.13), we obtain the following identity:

$$
\prod_{i=1}^{\ell}\left(z_{i}^{\frac{1}{2}}+z_{i}^{-\frac{1}{2}}\right) \frac{\prod_{n \in \mathbb{N}}\left(1+x_{n} z_{i}\right)\left(1+x_{n} z_{i}^{-1}\right)}{\prod_{r \in \frac{1}{2}+\mathbb{Z}_{+}}\left(1-x_{r} z_{i}\right)\left(1-x_{r} z_{i}^{-1}\right)}=\sum_{\lambda \in \mathcal{P}(\operatorname{Pin}(2 \ell))} \operatorname{ch} L\left(\widehat{\mathcal{B}}, \bar{\Lambda}^{\mathfrak{b}}(\lambda)\right) \operatorname{ch} V_{\operatorname{Pin}(2 \ell)}^{\lambda} .
$$

Theorem 3.4. For $\lambda \in \mathcal{P}(\operatorname{Pin}(2 \ell))$, we have

$$
\operatorname{ch} L\left(\widehat{\mathcal{B}}, \bar{\Lambda}^{\mathfrak{b}}(\lambda)\right)=\frac{1}{\bar{D}^{\mathfrak{b}}} \sum_{k=0}^{\infty} \sum_{w \in W_{k}^{0}}(-1)^{k} H S_{\left(\lambda_{w}\right)^{\prime}}\left(\mathbf{x}_{>0}\right)
$$

where

$$
\bar{D}^{\mathfrak{b}}=\prod_{\substack{i, j \in \mathbb{N} \\ i \leq j}} \frac{\left(1-x_{i}\right)\left(1-x_{i} x_{j+1}\right)\left(1-x_{i-\frac{1}{2}} x_{j-\frac{1}{2}}\right)}{\left(1+x_{i-\frac{1}{2}}\right)\left(1+x_{i} x_{j+\frac{1}{2}}\right)\left(1+x_{i-\frac{1}{2}} x_{j}\right)}
$$

Proof. The proof is similar to that of Theorem 3.3. Replace $\left\{x_{j}\right\}_{j \in \mathbb{N}}$ with $\left\{x_{j}\right\}_{j \in \frac{1}{2} \mathbb{N}}$ in (2.14), and then apply the involution to the symmetric functions in $\left\{x_{j}\right\}_{j \in \frac{1}{2}+\mathbb{Z}_{+}}$ (cf. [M, Chapter I $\S 5$ Ex. 4,5]). Then the result follows from the linear independence of $\left\{\operatorname{ch} V_{\operatorname{Pin}(2 \ell)}^{\lambda} \mid \lambda \in \mathcal{P}(\operatorname{Pin}(2 \ell))\right\}$.

3.3.3. The $(\widehat{\mathcal{C}}, \operatorname{Sp}(2 \ell))$-duality. For $\lambda \in \mathcal{P}(\operatorname{Sp}(2 \ell))$, we define $\bar{\Lambda}^{\mathfrak{c}}(\lambda) \in \overline{\mathfrak{h}}^{*}$ by

$$
\begin{aligned}
& \bar{\Lambda}^{\mathfrak{c}}(\lambda)_{i}=\left\langle\lambda_{i}^{\prime}-i\right\rangle, \quad i \in \mathbb{N}, \\
& \bar{\Lambda}^{\mathfrak{c}}(\lambda)_{r}=\left\langle\lambda_{r+1 / 2}-(r-1 / 2)\right\rangle, \quad r \in \frac{1}{2}+\mathbb{Z}_{+}, \\
& \left\langle\bar{\Lambda}^{\mathfrak{c}}(\lambda), K\right\rangle=\ell .
\end{aligned}
$$

Proposition 3.3. [LZ, Theorem 5.3] There exists an action of $\widehat{\mathcal{C}} \times \operatorname{Sp}(2 \ell)$ on $\overline{\mathfrak{F}}_{0}^{\ell}$. Furthermore, under this joint action, we have

$$
\overline{\mathfrak{F}}_{0}^{\ell} \cong \bigoplus_{\lambda \in \mathcal{P}(\operatorname{Sp}(2 \ell))} L\left(\widehat{\mathrm{e}}, \bar{\Lambda}^{\mathfrak{c}}(\lambda)\right) \otimes V_{\mathrm{Sp}(2 \ell)}^{\lambda}
$$

Computing the trace of the operator $\prod_{s \in \frac{1}{2} \mathbb{N}} x_{s}^{\widetilde{E}_{s}} \prod_{i=1}^{\ell} z_{i}^{\widetilde{e}_{i}}$ on both sides of (3.15), we obtain the following:

$$
\prod_{i=1}^{\ell} \frac{\prod_{n \in \mathbb{N}}\left(1+x_{n} z_{i}\right)\left(1+x_{n} z_{i}^{-1}\right)}{\prod_{r \in \frac{1}{2}+\mathbb{Z}_{+}}\left(1-x_{r} z_{i}\right)\left(1-x_{r} z_{i}^{-1}\right)}=\sum_{\lambda \in \mathcal{P}(\operatorname{Sp}(2 \ell))} \operatorname{ch} L\left(\widehat{\mathcal{C}}, \bar{\Lambda}^{\mathfrak{c}}(\lambda)\right) \operatorname{ch} V_{\operatorname{Sp}(2 \ell)}^{\lambda} .
$$


Similar to Theorem 3.4, we obtain the following.

Theorem 3.5. For $\lambda \in \mathcal{P}(\operatorname{Sp}(2 \ell))$, we have

$$
\operatorname{ch} L\left(\widehat{\mathrm{e}}, \bar{\Lambda}^{\mathfrak{c}}(\lambda)\right)=\frac{1}{\bar{D}^{\mathfrak{c}}} \sum_{k=0}^{\infty} \sum_{w \in W_{k}^{0}}(-1)^{k} H S_{\left(\lambda_{w}\right)^{\prime}}\left(\mathbf{x}_{>0}\right),
$$

where

$$
\bar{D}^{\mathfrak{c}}=\prod_{\substack{i, j \in \mathbb{N} \\ i \leq j}} \frac{\left(1-x_{i} x_{j}\right)\left(1-x_{i-\frac{1}{2}} x_{j+\frac{1}{2}}\right)}{\left(1+x_{i} x_{j+\frac{1}{2}}\right)\left(1+x_{i-\frac{1}{2}} x_{j}\right)}
$$

3.3.4. The $(\widehat{\mathcal{D}}, \mathrm{O}(m))$-duality. For $\lambda \in \mathcal{P}(\mathrm{O}(m))$, we define $\bar{\Lambda}^{\mathfrak{d}}(\lambda) \in \overline{\mathfrak{h}}^{*}$ by

$$
\begin{aligned}
& \bar{\Lambda}^{\mathfrak{d}}(\lambda)_{i}=\left\langle\lambda_{i}^{\prime}-i\right\rangle, \quad i \in \mathbb{N}, \\
& \bar{\Lambda}^{\mathfrak{d}}(\lambda)_{r}=\left\langle\lambda_{r+1 / 2}-(r-1 / 2)\right\rangle, \quad r \in \frac{1}{2}+\mathbb{Z}_{+}, \\
& \left\langle\bar{\Lambda}^{\mathfrak{d}}(\lambda), K\right\rangle=\frac{m}{2} .
\end{aligned}
$$

Proposition 3.4. [LZ, Theorems 5.3 and 5.4] There exists an action of $\widehat{\mathcal{D}} \times \mathrm{O}(m)$ on $\overline{\mathfrak{F}}_{0}^{\frac{m}{2}}$. Furthermore, under this joint action, we have

$$
\overline{\mathfrak{F}}_{0}^{\frac{m}{2}} \cong \bigoplus_{\lambda \in \mathcal{P}(\mathrm{O}(m))} L\left(\widehat{\mathcal{D}}, \bar{\Lambda}^{\mathfrak{d}}(\lambda)\right) \otimes V_{\mathrm{O}(m)}^{\lambda}
$$

Suppose that $m=2 \ell$. Computing the trace of the operator $\prod_{s \in \frac{1}{2} \mathbb{N}} x_{s}^{\widetilde{E}_{s}} \prod_{i=1}^{\ell} z_{i}^{\tilde{e}_{i}}$ on both sides of (3.17), we obtain the following identity:

$$
\prod_{i=1}^{\ell} \frac{\prod_{n \in \mathbb{N}}\left(1+x_{n} z_{i}\right)\left(1+x_{n} z_{i}^{-1}\right)}{\prod_{r \in \frac{1}{2}+\mathbb{Z}_{+}}\left(1-x_{r} z_{i}\right)\left(1-x_{r} z_{i}^{-1}\right)}=\sum_{\lambda \in \mathcal{P}(\mathrm{O}(2 \ell))} \operatorname{ch} L\left(\widehat{\mathcal{D}}, \bar{\Lambda}^{\mathfrak{d}}(\lambda)\right) \operatorname{ch} V_{\mathrm{O}(2 \ell)}^{\lambda} .
$$

Suppose that $m=2 \ell+1$. Let $\epsilon$ be the eigenvalue of $-I_{m}$ on $\mathrm{O}(2 \ell+1)$-modules satisfying $\epsilon^{2}=1$. Calculating the trace of the operator $\prod_{s \in \frac{1}{2} \mathbb{N}} x_{s}^{\widetilde{E}_{s}} \prod_{i=1}^{\ell} z_{i}^{\tilde{e}_{i}}\left(-I_{m}\right)$ on (3.17) yields

$$
\prod_{i=1}^{\ell} \frac{\prod_{n \in \mathbb{N}}\left(1+\epsilon x_{n} z_{i}\right)\left(1+\epsilon x_{n} z_{i}^{-1}\right)\left(1+\epsilon x_{n}\right)}{\prod_{r \in \frac{1}{2}+\mathbb{Z}_{+}}\left(1-\epsilon x_{r} z_{i}\right)\left(1-\epsilon x_{r} z_{i}^{-1}\right)\left(1-\epsilon x_{r}\right)}=\sum_{\lambda \in \mathcal{P}(\mathrm{O}(2 \ell+1))} \operatorname{ch} L\left(\widehat{\mathcal{D}}, \bar{\Lambda}^{\mathfrak{d}}(\lambda)\right) \operatorname{ch} V_{\mathrm{O}(2 \ell+1)}^{\lambda} .
$$

Theorem 3.6. Suppose that $\lambda \in \mathcal{P}(\mathrm{O}(m))$ is given. Put

$$
\bar{D}^{\mathfrak{d}}=\prod_{\substack{i, j \in \mathbb{N} \\ i \leq j}} \frac{\left(1-x_{i} x_{j+1}\right)\left(1-x_{i-\frac{1}{2}} x_{j-\frac{1}{2}}\right)}{\left(1+x_{i} x_{j+\frac{1}{2}}\right)\left(1+x_{i-\frac{1}{2}} x_{j}\right)} .
$$


(1) If $m=2 \ell+1$, then we have

$$
\operatorname{ch} L\left(\widehat{\mathcal{D}}, \bar{\Lambda}^{\mathfrak{d}}(\lambda)\right)=\frac{1}{\bar{D}^{\mathfrak{d}}} \sum_{k=0}^{\infty} \sum_{w \in W_{k}^{0}}(-1)^{k} H S_{\left(\lambda_{w}\right)^{\prime}}\left(\mathbf{x}_{>0}\right) .
$$

(2) If $m=2 \ell$, then we have

$$
\begin{aligned}
& \operatorname{ch} L\left(\widehat{\mathcal{D}}, \bar{\Lambda}^{\mathfrak{d}}(\lambda)\right)+\operatorname{ch} L\left(\widehat{\mathcal{D}}, \bar{\Lambda}^{\mathfrak{d}}(\tilde{\lambda})\right) \\
& =\frac{1}{\bar{D}^{\mathfrak{d}}} \sum_{k=0}^{\infty} \sum_{w \in W_{k}^{0}}(-1)^{k}\left[H S_{\left(\lambda_{w}\right)^{\prime}}\left(\mathbf{x}_{>0}\right)+H S_{\left(\tilde{\lambda}_{w}\right)^{\prime}}\left(\mathbf{x}_{>0}\right)\right] .
\end{aligned}
$$

Proof. The proof is similar to that of Theorem 3.4. However, note that in the case of (2), $V_{\mathrm{O}(2 \ell)}^{\lambda}$ and $V_{\mathrm{O}(2 \ell)}^{\tilde{\lambda}}$ are isomorphic as $\mathfrak{s o}(2 \ell)$-modules, and they give the same character. Hence, the result follows from comparing the coefficients of $\operatorname{ch} V_{\mathrm{O}(2 \ell)}^{\lambda}$ for $\lambda \in \mathcal{P}(\mathrm{O}(2 \ell))$ with length less than or equal to $\ell$ (cf. [CZ2, Lemma 6.1]).

From now on we mean by $(\overline{\mathfrak{g}}, G)$ one of the dual pairs of Section [3.3, and by $\mathfrak{x} \in\{\mathfrak{a}, \mathfrak{b}, \mathfrak{c}, \mathfrak{d}\}$ the type of $\overline{\mathfrak{g}}$. The map (consisting of involutions of symmetric functions) that transforms (2.18) into $\operatorname{ch} L\left(\overline{\mathfrak{g}}, \Lambda^{\mathfrak{x}}(\lambda)\right)$ of the form in Theorems 3.3 3.6 will be denoted by $\omega^{\mathfrak{x}}$.

\section{The Casimir operators}

4.1. The bilinear form $(\cdot \mid \cdot)_{c}$ and the Casimir operator $\Omega$ of $\mathfrak{g}$. Suppose first that $\mathfrak{g}=\widehat{\mathfrak{g l}}_{\infty}$. We fix a symmetric bilinear form $(\cdot \mid \cdot)_{c}$ on $\mathfrak{h}^{*}$ satisfying

$$
\begin{aligned}
& \left(\lambda \mid \epsilon_{i}\right)_{c}=\left\langle\lambda, E_{i i}-\zeta(i) \frac{K}{2}\right\rangle, \quad \lambda \in \mathfrak{h}^{*}, i \in \mathbb{Z}, \\
& \left(\Lambda_{0}^{\mathfrak{a}} \mid \Lambda_{0}^{\mathfrak{a}}\right)_{c}=\left(\Lambda_{0}^{\mathfrak{a}} \mid \rho_{c}\right)_{c}=0
\end{aligned}
$$

where $\zeta(i)=1$ (resp. -1 ) if $i>0$ (resp. $i \leq 0$ ). Such a form exists, since the vectors $\left\{\epsilon_{i}\right\}_{i \in \mathbb{Z}}, \Lambda_{0}^{\mathfrak{a}}$ and $\rho_{c}$ are linearly independent. We check easily that

$$
\begin{aligned}
& \left(\epsilon_{i} \mid \epsilon_{j}\right)_{c}=\delta_{i j}, \quad\left(\Lambda_{0}^{\mathfrak{a}} \mid \epsilon_{i}\right)_{c}=-\frac{\zeta(i)}{2}, \quad i, j \in \mathbb{Z}, \\
& \left(\rho_{c} \mid \alpha_{i}\right)_{c}=\frac{1}{2}\left(\alpha_{i} \mid \alpha_{i}\right)_{c}, \quad i \in I .
\end{aligned}
$$

Suppose that $\mathfrak{g}=\mathfrak{x}_{\infty}$ with $\mathfrak{x} \in\{\mathfrak{b}, \mathfrak{c}, \mathfrak{d}\}$. We choose a symmetric bilinear form $(\cdot \mid \cdot)_{c}$ on $\mathfrak{h}^{*}$ satisfying

$$
\begin{aligned}
& \left(\lambda \mid \epsilon_{i}\right)_{c}=\left\langle\lambda, \widetilde{E}_{i}-K\right\rangle, \quad i \in \mathbb{N}, \\
& \left(\Lambda_{0}^{\mathfrak{x}} \mid \Lambda_{0}^{\mathfrak{x}}\right)_{c}=\left(\Lambda_{0}^{\mathfrak{x}} \mid \rho_{c}\right)_{c}=0 .
\end{aligned}
$$

Also one checks that $\left(\epsilon_{i} \mid \epsilon_{j}\right)_{c}=\delta_{i j},\left(\Lambda_{0}^{\mathfrak{x}} \mid \epsilon_{i}\right)_{c}=-r$ for $i, j \in \mathbb{N}$, where $r=\frac{1}{2}, 1, \frac{1}{2}$ for $\mathfrak{x}=\mathfrak{b}, \mathfrak{c}, \mathfrak{d}$, respectively, and $2\left(\rho_{c} \mid \alpha_{i}\right)_{c}=\left(\alpha_{i} \mid \alpha_{i}\right)_{c}$ for $i \in I$. 
Now for each $\mathfrak{g}$ of type $\mathfrak{x} \in\{\mathfrak{a}, \mathfrak{b}, \mathfrak{c}, \mathfrak{d}\}$, let $\left\{s_{i}^{\mathfrak{x}}\right\}_{i \in I}$ be the sequence defined by

$$
s_{i}^{\mathfrak{a}}=s_{i}^{\mathfrak{d}}=1, \quad i \in I, \quad s_{i}^{\mathfrak{b}}=\left\{\begin{array}{ll}
\frac{1}{2}, & \text { if } i=0, \\
1, & \text { if } i \geq 1,
\end{array} \quad s_{i}^{\mathfrak{c}}= \begin{cases}2, & \text { if } i=0, \\
1, & \text { if } i \geq 1 .\end{cases}\right.
$$

Then it follows that

$$
\left(\lambda \mid \alpha_{i}\right)_{c}=s_{i}^{\mathfrak{x}}\left\langle\lambda, \alpha_{i}^{\vee}\right\rangle
$$

for $\lambda \in \mathfrak{h}^{*}, i \in I$ so that $\left(\alpha_{i} \mid \alpha_{j}\right)_{c}=s_{j}^{\mathfrak{x}}\left\langle\alpha_{i}, \alpha_{j}^{\vee}\right\rangle$, for $i, j \in I$. By defining $\left(\alpha_{i}^{\vee} \mid \alpha_{j}^{\vee}\right)_{c}:=$ $\left(s_{i}^{\mathfrak{x}} s_{j}^{\mathfrak{x}}\right)^{-1}\left(\alpha_{i} \mid \alpha_{j}\right)_{c}$, we obtain a symmetric bilinear form on the Cartan subalgebra of $\mathfrak{g}^{\prime}=[\mathfrak{g}, \mathfrak{g}]$, which can be extended to a non-degenerate invariant symmetric bilinear form on $\mathfrak{g}^{\prime}$ such that

$$
\left(e_{i} \mid f_{j}\right)_{c}=\delta_{i j} / s_{i}^{\mathfrak{x}}
$$

where $e_{i}$ and $f_{j}(i, j \in I)$ denote the Chevalley generators of $\mathfrak{g}^{\prime}$ with $\left[e_{i}, f_{i}\right]=$ $\alpha_{i}^{\vee}$. Thus the symmetric bilinear form on $\mathfrak{h}^{*}$ induces an invariant symmetric nondegenerate bilinear form on the derived subalgebra of $\mathfrak{g}$. Since every root space is one-dimensional, we can choose a basis $\left\{u_{\alpha}\right\}$ of $\mathfrak{g}_{\alpha}$ for $\alpha \in \Delta^{+}$and a dual basis $\left\{u^{\alpha}\right\}$ of $\mathfrak{g}_{-\alpha}$ with respect to $(\cdot \mid \cdot)_{c}$.

Let $V$ be a highest weight $\mathfrak{g}$-module with weight space decomposition $V=\oplus_{\mu} V_{\mu}$. Define $\Gamma_{1}: V \rightarrow V$ to be the linear map that acts as the scalar $\left(\mu+2 \rho_{c} \mid \mu\right)_{c}$ on $V_{\mu}$. Let $\Gamma_{2}:=2 \sum_{\alpha \in \Delta^{+}} u^{\alpha} u_{\alpha}$. Define the Casimir operator (cf. [J]) to be

$$
\Omega:=\Gamma_{1}+\Gamma_{2} .
$$

It follows from (4.1) and (4.2) that $\Omega$ commutes with the action of $\mathfrak{g}$ on $V$ (cf. [J], Proposition 3.6]). Thus, if $V$ is generated by a highest weight vector with highest weight $\lambda$, then $\Omega$ acts on $V$ as the scalar $\left(\lambda+2 \rho_{c} \mid \lambda\right)_{c}$.

4.2. The bilinear form $(\cdot \mid \cdot)_{s}$ and the Casimir operator $\bar{\Omega}$ of $\overline{\mathfrak{g}}$. Suppose first that $\overline{\mathfrak{g}}=\widehat{\mathfrak{g l}}_{\infty \mid \infty}$. Define $\bar{\Lambda}_{0}^{\mathfrak{a}} \in \overline{\mathfrak{h}}^{*}$ by $\left\langle\bar{\Lambda}_{0}^{\mathfrak{a}}, K\right\rangle=1$ and $\left\langle\bar{\Lambda}_{0}^{\mathfrak{a}}, E_{r r}\right\rangle=0$, for all $r \in \frac{1}{2} \mathbb{Z}$. We choose a symmetric bilinear form $(\cdot \mid \cdot)_{s}$ on $\overline{\mathfrak{h}}^{*}$ satisfying

$$
\begin{aligned}
& \left(\lambda \mid \delta_{r}\right)_{s}=(-1)^{2 r}\left\langle\lambda, E_{r r}-(-1)^{2 r} \zeta(r) \frac{K}{2}\right\rangle, \quad \lambda \in \overline{\mathfrak{h}}^{*}, r \in \frac{1}{2} \mathbb{Z}, \\
& \left(\bar{\Lambda}_{0}^{\mathfrak{a}} \mid \bar{\Lambda}_{0}^{\mathfrak{a}}\right)_{s}=0 .
\end{aligned}
$$

Here we set that $\rho_{s}=0 \in \overline{\mathfrak{h}}^{*}$. We check easily that

$$
\begin{aligned}
& \left(\delta_{r} \mid \delta_{t}\right)_{s}=(-1)^{2 r} \delta_{r t}, \quad\left(\bar{\Lambda}_{0}^{\mathfrak{a}} \mid \delta_{r}\right)_{s}=-\frac{\zeta(r)}{2}, \quad r, t \in \frac{1}{2} \mathbb{Z}, \\
& \left(\rho_{s} \mid \beta_{r}\right)_{s}=\frac{1}{2}\left(\beta_{r} \mid \beta_{r}\right)_{s}, \quad r \in \bar{I} .
\end{aligned}
$$


Suppose that $\overline{\mathfrak{g}}=\widehat{X}$ for $X \in\{\mathcal{B}, \mathcal{C}, \mathcal{D}\}$, with $\mathfrak{x} \in\{\mathfrak{b}, \mathfrak{c}, \mathfrak{d}\}$ denoting the respective type of $\overline{\mathfrak{g}}$. Define $\bar{\Lambda}_{0}^{\mathfrak{x}} \in \overline{\mathfrak{h}}^{*}$ by $\left\langle\bar{\Lambda}_{0}^{\mathfrak{x}}, K\right\rangle=1$ and $\left\langle\bar{\Lambda}_{0}^{\mathfrak{x}}, \widetilde{E}_{r}\right\rangle=0$ for $r \in \frac{1}{2} \mathbb{N}$. For $\widehat{\mathfrak{C}}$, we put $\rho_{s}=0$. For the other cases, let $\rho_{s} \in \overline{\mathfrak{h}}^{*}$ be determined by

$$
\begin{aligned}
& \left\langle\rho_{s}, \widetilde{E}_{r}\right\rangle=\left\{\begin{array}{ll}
(-1)^{2 r} \frac{1}{2}, & \text { for } \widehat{\mathcal{B}}, \\
(-1)^{2 r}, & \text { for } \widehat{\mathcal{D}},
\end{array} \quad r \in \frac{1}{2} \mathbb{N},\right. \\
& \left\langle\rho_{s}, K\right\rangle=0 .
\end{aligned}
$$

We fix a symmetric bilinear form $(\cdot \mid \cdot)_{s}$ on $\overline{\mathfrak{h}}^{*}$ satisfying

$$
\begin{aligned}
& \left(\lambda \mid \delta_{r}\right)_{s}=(-1)^{2 r}\left\langle\lambda, \widetilde{E}_{r}-(-1)^{2 r} K\right\rangle, \quad \lambda \in \overline{\mathfrak{h}}^{*}, r \in \frac{1}{2} \mathbb{N}, \\
& \left(\bar{\Lambda}_{0}^{\mathfrak{x}} \mid \bar{\Lambda}_{0}^{\mathfrak{x}}\right)_{s}=\left(\bar{\Lambda}_{0}^{\mathfrak{x}} \mid \rho_{s}\right)_{s}=0 .
\end{aligned}
$$

Then we can check that $\left(\delta_{r} \mid \delta_{t}\right)_{s}=(-1)^{2 r} \delta_{r t},\left(\bar{\Lambda}_{0}^{\mathfrak{x}} \mid \delta_{r}\right)_{s}=-1$ for $r, t \in \frac{1}{2} \mathbb{N}$, and $2\left(\rho_{s} \mid \beta_{r}\right)_{s}=\left(\beta_{r} \mid \beta_{r}\right)_{s}$ for $r \in \bar{I}$.

Now, for each $\overline{\mathfrak{g}}$ of type $\mathfrak{x} \in\{\mathfrak{a}, \mathfrak{b}, \mathfrak{c}, \mathfrak{d}\}$, let $\left\{\bar{s}_{r}^{\mathfrak{r}}\right\}_{r \in \bar{I}}$ be the sequence defined by

$$
\begin{aligned}
& \bar{s}_{r}^{\mathfrak{a}}=\bar{s}_{r}^{\mathfrak{b}}=\bar{s}_{r}^{\mathfrak{c}}=(-1)^{2 r}, \quad r \in \bar{I}, \\
& \bar{s}_{r}^{\mathfrak{d}}= \begin{cases}2, & \text { if } r=0, \\
(-1)^{2 r}, & \text { if } r \geq \frac{1}{2} .\end{cases}
\end{aligned}
$$

Then we have

$$
\left(\lambda \mid \beta_{r}\right)_{s}=\bar{s}_{r}^{\mathfrak{x}}\left\langle\lambda, \beta_{r}^{\vee}\right\rangle, \quad \lambda \in \overline{\mathfrak{h}}^{*}, r \in \bar{I},
$$

so that $\left(\beta_{r} \mid \beta_{t}\right)_{s}=\bar{s}_{t}^{\mathfrak{x}}\left\langle\beta_{r}, \beta_{t}^{\vee}\right\rangle$ for $r, t \in \bar{I}$. By defining $\left(\beta_{r}^{\vee} \mid \beta_{t}^{\vee}\right)_{s}:=\left(\bar{s}_{r}^{\mathfrak{r}} \bar{s}_{t}^{\mathfrak{r}}\right)^{-1}\left(\beta_{r} \mid \beta_{t}\right)_{s}$, we obtain a symmetric bilinear form on the Cartan subalgebra of $\overline{\mathfrak{g}}^{\prime}=[\overline{\mathfrak{g}}, \overline{\mathfrak{g}}]$, which can be extended to a non-degenerate invariant super-symmetric bilinear form on $\overline{\mathfrak{g}}^{\prime}$ such that

$$
\left(\bar{e}_{r} \mid \bar{f}_{t}\right)_{s}=\delta_{r t} / \bar{s}_{r}^{\mathfrak{x}}
$$

where $\bar{e}_{r}$ and $\bar{f}_{t}(r, t \in \bar{I})$ denote the Chevalley generators of $\overline{\mathfrak{g}}$ with $\left[\bar{e}_{r}, \bar{f}_{r}\right]=\beta_{r}^{\vee}$.

We have now all the ingredients to define the super-analogue of the Casimir operator. Namely, for $\beta \in \bar{\Delta}^{+}$, let $\overline{\mathfrak{g}}_{\beta}$ be the root space of $\overline{\mathfrak{g}}$ corresponding to $\beta$. Take a basis $\left\{u_{\beta}\right\}$ of $\overline{\mathfrak{g}}_{\beta}$, and a dual basis $\left\{u^{\beta}\right\}$ of $\overline{\mathfrak{g}}_{-\beta}$ with respect to $(\cdot \mid \cdot)_{s}$. For any highest weight $\overline{\mathfrak{g}}$-module $V$, with weight space decomposition $V=\oplus_{\mu} V_{\mu}$, we define $\bar{\Gamma}_{1}: V \rightarrow V$ to be the linear map that acts as the scalar $\left(\mu+2 \rho_{s} \mid \mu\right)_{s}$ on $V_{\mu}$. Let $\bar{\Gamma}_{2}:=2 \sum_{\beta \in \bar{\Delta}^{+}} u^{\beta} u_{\beta}$. For example, if $\overline{\mathfrak{g}}=\widehat{\mathfrak{g l}}_{\infty \mid \infty}$, then we have for $r \in \bar{I}$

$$
u^{\beta_{r}} u_{\beta_{r}}=(-1)^{2 r} E_{r+\frac{1}{2}, r} E_{r, r+\frac{1}{2}} .
$$


Define the Casimir operator to be

$$
\bar{\Omega}:=\bar{\Gamma}_{1}+\bar{\Gamma}_{2} .
$$

Proposition 4.1. Let $V$ be a highest weight $\overline{\mathfrak{g}}$-module. The operator $\bar{\Omega}$ commutes with the action of $\overline{\mathfrak{g}}$ on $V$. In particular, if $V$ is generated by a highest weight vector with highest weight $\lambda$, then $\bar{\Omega}$ acts on $V$ as the scalar $\left(\lambda+2 \rho_{s} \mid \lambda\right)_{s}$.

Proof. The argument is parallel to the one given to prove [J, Proposition 3.6]. Here we use (4.3) and (4.4) to replace the corresponding identities of [J].

4.3. $\overline{\mathfrak{u}}_{-}$-homology groups of $\overline{\mathfrak{g}}$-modules. Suppose that $(\mathfrak{g}, G)$ and $(\overline{\mathfrak{g}}, G)$ are the dual pairs of type $\mathfrak{x} \in\{\mathfrak{a}, \mathfrak{b}, \mathfrak{c}, \mathfrak{d}\}$ given in Section 2.3 and Section 3.3. and $\lambda \in$ $\mathcal{P}(G)$. Recall the following results for integrable modules of generalized Kac-Moody algebras [J, L] applied to our setting.

\section{Proposition 4.2.}

(i) If $\eta \in \mathcal{P}_{\mathfrak{l}}^{+}$is a weight in $\Lambda^{k} \mathfrak{u}_{-} \otimes L\left(\mathfrak{g}, \Lambda^{\mathfrak{x}}(\lambda)\right)$ with $\left(\eta+2 \rho_{c} \mid \eta\right)_{c}=\left(\Lambda^{\mathfrak{x}}(\lambda)+\right.$ $\left.2 \rho_{c} \mid \Lambda^{\mathfrak{x}}(\lambda)\right)_{c}$, then there exists $w \in W_{k}^{0}$ with $\eta=w \circ \Lambda^{\mathfrak{x}}(\lambda)$ and $\eta$ appears with multiplicity one.

(ii) The $\mathfrak{l}$-module $\mathrm{H}_{k}\left(\mathfrak{u}_{-} ; L\left(\mathfrak{g}, \Lambda^{\mathfrak{x}}(\lambda)\right)\right)$ is completely reducible. Furthermore if $L(\mathfrak{l}, \eta)$ is an irreducible component of $\mathrm{H}_{k}\left(\mathfrak{u}_{-} ; L\left(\mathfrak{g}, \Lambda^{\mathfrak{x}}(\lambda)\right)\right)$, then $\left(\eta+2 \rho_{c} \mid \eta\right)_{c}=$ $\left(\Lambda^{\mathfrak{x}}(\lambda)+2 \rho_{c} \mid \Lambda^{\mathfrak{x}}(\lambda)\right)_{c}$.

Let $\bar{\Delta}:=\bar{\Delta}^{+} \cup \bar{\Delta}^{-}$be the set of roots of $\overline{\mathfrak{g}}$, where $\bar{\Delta}^{-}=-\bar{\Delta}^{+}$. Let $\bar{\Delta}_{S}^{ \pm}:=$ $\bar{\Delta}^{ \pm} \cap\left(\sum_{r \neq 0} \mathbb{Z} \beta_{r}\right)$ and $\bar{\Delta}^{ \pm}(S):=\bar{\Delta}^{ \pm} \backslash \bar{\Delta}_{S}^{ \pm}$. Let

$$
\overline{\mathfrak{u}}_{ \pm}:=\sum_{\beta \in \bar{\Delta}^{ \pm}(S)} \overline{\mathfrak{g}}_{\beta}, \quad \overline{\mathfrak{l}}:=\sum_{\beta \in \bar{\Delta}_{S}^{+} \cup \bar{\Delta}_{S}^{-}} \overline{\mathfrak{g}}_{\beta} \oplus \overline{\mathfrak{h}}, \quad \overline{\mathfrak{p}}:=\overline{\mathfrak{l}} \oplus \overline{\mathfrak{u}}_{+} .
$$

Then we have $\overline{\mathfrak{g}}=\overline{\mathfrak{u}}_{+} \oplus \overline{\mathfrak{l}} \oplus \overline{\mathfrak{u}}_{-}$. The Lie superalgebras $\overline{\mathfrak{l}}$ and $\overline{\mathfrak{g}}$ share the same Cartan subalgebra. It is not difficult to see that $\overline{\mathfrak{l}}=\overline{\mathfrak{g l}}_{>0} \oplus \overline{\mathfrak{g l}}_{\leq 0} \oplus \mathbb{C} K$ if $\overline{\mathfrak{g}}=\widehat{\mathfrak{g l}}_{\infty \mid \infty}$, and $\overline{\mathfrak{l}} \cong \overline{\mathfrak{g l}}_{>0} \oplus \mathbb{C} K$ otherwise.

For $\mu \in \overline{\mathfrak{h}}^{*}$ we denote by $L(\overline{\mathfrak{l}}, \mu)$ the irreducible highest weight representation of $\overline{\mathfrak{l}}$ with highest weight $\mu$. For $\overline{\mathfrak{g}}=\widehat{\mathfrak{g l}}_{\infty \mid \infty}$, let $\mathcal{P}_{\overline{\mathfrak{l}}}^{+}$be the set of $\mu \in \overline{\mathfrak{h}}^{*}$ such that $\mu=\sum_{r \in \frac{1}{2} \mathbb{Z}} \mu_{r} \delta_{r}+c \bar{\Lambda}_{0}^{\mathfrak{x}}$ for some $c \in \mathbb{C}$ and $\sum_{r \in \frac{1}{2} \mathbb{N}} \mu_{r} \delta_{r}$ (resp. $\sum_{r \in-\frac{1}{2} \mathbb{Z}_{+}} \mu_{r} \delta_{r}$ ) is a highest weight for an irreducible (resp. irreducible dual) tensor representation of $\overline{\mathfrak{g l}}_{>0}\left(\right.$ resp. $\left.\overline{\mathfrak{g l}}_{\leq 0}\right)$. For $\overline{\mathfrak{g}}=\widehat{X}$ with $X \in\{\mathcal{B}, \mathcal{C}, \mathcal{D}\}$, let $\mathcal{P}_{\overline{\mathfrak{l}}}^{+}$be the set of $\mu \in \overline{\mathfrak{h}}^{*}$ such

that $\mu=\sum_{r \in \frac{1}{2} \mathbb{N}} \mu_{r} \delta_{r}+c \bar{\Lambda}_{0}^{\mathfrak{x}}$ for some $c \in \mathbb{C}$ and $\sum_{r \in \frac{1}{2} \mathbb{N}} \mu_{r} \delta_{r}$ is a highest weight for an irreducible tensor representation of $\overline{\mathfrak{g l}}_{>0}$.

Now consider the homology groups $\mathrm{H}_{k}\left(\overline{\mathfrak{u}}_{-} ; L\left(\overline{\mathfrak{g}}, \bar{\Lambda}^{\mathfrak{x}}(\lambda)\right)\right)$, which are defined analogously (see e.g. [Fu, $\mathrm{KK}]$ ). 
Lemma 4.1. The $\overline{\mathfrak{l}}$-module $L\left(\overline{\mathfrak{g}}, \bar{\Lambda}^{\mathfrak{x}}(\lambda)\right)$ is completely reducible.

Proof. We will only show this for $\overline{\mathfrak{g}}=\widehat{\mathfrak{g l}}_{\infty \mid \infty}$. The other cases are analogous and omitted.

Consider the Fock space $\overline{\mathfrak{F}}^{\ell}$ with $\overline{\mathfrak{l}} \times \mathrm{GL}(\ell)$-character

$$
\prod_{i=1}^{\ell} \frac{\prod_{n \in \mathbb{N}}\left(1+x_{n} z_{i}\right)\left(1+x_{-n+1}^{-1} z_{i}^{-1}\right)}{\prod_{r \in 1 / 2+\mathbb{Z}_{+}}\left(1-x_{r} z_{i}\right)\left(1-x_{-r}^{-1} z_{i}^{-1}\right)},
$$

which is the left hand side of (3.10). Using (3.12) twice we see that (4.7) can be written as

$$
\sum_{\lambda, \mu \in \mathcal{P}^{+} ; l(\lambda), l(\mu) \leq \ell} H S_{\lambda^{\prime}}\left(\mathbf{x}_{>0}\right) H S_{\mu}\left(\mathbf{x}_{\leq 0}^{-1}\right) s_{\lambda}\left(z_{1}, \cdots, z_{\ell}\right) s_{\mu}\left(z_{1}^{-1}, \cdots, z_{\ell}^{-1}\right) .
$$

Thus (4.7) can be written as an infinite sum of $H S_{\lambda^{\prime}}\left(\mathbf{x}_{>0}\right) H S_{\mu}\left(\mathbf{x}_{\leq 0}^{-1}\right)$ such that each summand has a finite multiplicity. Now Theorem 3.2 implies that $\overline{\mathfrak{F}} \ell$, as an $\overline{\mathfrak{l}}$-module, is completely reducible. Since $L\left(\overline{\mathfrak{g}}, \bar{\Lambda}^{\mathfrak{a}}(\lambda)\right)$ is a direct summand of a completely reducible module, it is also completely reducible.

Lemma 4.2. The $\overline{\mathfrak{l}}-$ module $\mathrm{H}_{k}\left(\overline{\mathfrak{u}}_{-} ; L\left(\overline{\mathfrak{g}}, \bar{\Lambda}^{\mathfrak{x}}(\lambda)\right)\right)$ is completely reducible.

Proof. First suppose that $\overline{\mathfrak{g}}=\widehat{\mathfrak{g l}}_{\infty \mid \infty}$ with $\overline{\mathfrak{l}}=\overline{\mathfrak{g l}}_{\leq 0} \oplus \overline{\mathfrak{g l}}_{>0} \oplus \mathbb{C} K$. As a $\overline{\mathfrak{g l}}_{\leq 0} \oplus \overline{\mathfrak{g l}}_{>0^{-}}$ module, $\overline{\mathfrak{u}}_{-} \cong \mathbb{C}^{\leq 0 *} \otimes \mathbb{C}^{>0}$. We claim that $\Lambda^{k}\left(\overline{\mathfrak{u}}_{-}\right)$is completely reducible, and each irreducible component is an irreducible dual tensor representation of $\overline{\mathfrak{g l}}_{\leq 0}$ tensored with an irreducible tensor representation of $\overline{\mathfrak{g l}}_{>0}$. This can be proved, for example, by using an analogous truncation functor argument as in Section 3.2 .2 in combination with the skew-symmetric Howe duality for a pair of finite-dimensional general Lie superalgebras [CW1, Theorem 3.3]. By Lemma 4.1 $L\left(\widehat{\mathfrak{g l}}_{\infty \mid \infty}, \bar{\Lambda}^{\mathfrak{a}}(\lambda)\right)$ as an $\overline{\mathfrak{l}}$-module is completely reducible, and hence by Theorem $3.2 \Lambda^{k}\left(\overline{\mathfrak{u}}_{-}\right) \otimes L\left(\left.\widehat{\mathfrak{g l}}_{\infty}\right|_{\infty}, \bar{\Lambda}^{\mathfrak{a}}(\lambda)\right)$ is a completely reducible $\overline{\mathfrak{l}}$-module. Since any subquotient of a completely reducible module is also completely reducible, the result follows.

Now suppose that $\overline{\mathfrak{g}}=\widehat{\mathcal{B}}, \widehat{\mathfrak{C}}, \widehat{\mathcal{D}}$. Then $\overline{\mathfrak{l}}$ is isomorphic to $\overline{\mathfrak{g l}}_{>0} \oplus \mathbb{C} K$ and as a $\overline{\mathfrak{g l}}_{>0}$-module,

$$
\overline{\mathfrak{u}}_{-} \cong \begin{cases}\Lambda^{2}\left(\mathbb{C}^{>0}\right) \oplus \mathbb{C}^{>0}, & \text { if } \overline{\mathfrak{g}}=\widehat{\mathcal{B}} \\ S^{2}\left(\mathbb{C}^{>0}\right), & \text { if } \overline{\mathfrak{g}}=\widehat{\mathfrak{C}}, \\ \Lambda^{2}\left(\mathbb{C}^{>0}\right), & \text { if } \overline{\mathfrak{g}}=\widehat{\mathcal{D}},\end{cases}
$$

which is a direct sum of irreducible tensor representations. By Theorem $3.2 \Lambda^{k}\left(\overline{\mathfrak{u}}_{-}\right)$ is completely reducible over $\overline{\mathfrak{l}}$. This together with Lemma 4.1 shows that $\Lambda^{k}\left(\overline{\mathfrak{u}}_{-}\right) \otimes$ $L\left(\overline{\mathfrak{g}}, \bar{\Lambda}^{\mathfrak{x}}(\lambda)\right)$, and hence $\mathrm{H}_{k}\left(\overline{\mathfrak{u}}_{-} ; L\left(\overline{\mathfrak{g}}, \bar{\Lambda}^{\mathfrak{x}}(\lambda)\right)\right)$, is completely reducible. 
Remark 4.1. Alternatively, Lemmas 4.1 and 4.2 also follow from the unitarity of the respective $\overline{\mathfrak{l}}$-modules.

We have the following super-analogue of the action of the Casimir operator on homology groups (cf. [GL, L, J]).

Proposition 4.3. Let $\gamma \in \mathcal{P}_{\overline{\mathfrak{l}}}^{+}$and let $L(\overline{\mathfrak{l}}, \gamma)$ be the irreducible $\overline{\mathfrak{l}}$-module of highest weight $\gamma$. If $L(\overline{\mathfrak{l}}, \gamma)$ is a component of $\mathrm{H}_{k}\left(\overline{\mathfrak{u}}_{-} ; L\left(\overline{\mathfrak{g}}, \bar{\Lambda}^{\mathfrak{x}}(\lambda)\right)\right)$, then $\left(\gamma+2 \rho_{s} \mid \gamma\right)_{s}=$ $\left(\bar{\Lambda}^{\mathfrak{x}}(\lambda)+2 \rho_{s} \mid \bar{\Lambda}^{\mathfrak{x}}(\lambda)\right)_{s}$.

Proof. This can be proved following the same type of arguments as the one given in the proof of [L, Proposition 18] and thus we will omit the details. We only remark that in the process we use the same bilinear form $(\cdot \mid \cdot)_{s}$ and the same $\rho_{s}$ to define the corresponding Casimir operator for $\overline{\mathfrak{l}}$ as in (4.5).

\section{Computation of $\overline{\mathfrak{u}}_{-}$-homology Groups}

5.1. Comparison of Casimir eigenvalues. Let $\overline{\mathcal{P}}^{1}$ denote the set of sequences $a=\left(a_{\frac{1}{2}}, a_{1}, a_{\frac{3}{2}}, \cdots\right)$, where $\left(a_{\frac{1}{2}}, a_{\frac{3}{2}}, \cdots\right)$ and $\left(a_{1}, a_{2}, \cdots\right)$ are strict partitions, such that $a_{s}=0$ implies that $a_{s+\frac{1}{2}}=0$, for all $s$. We can define a map $\theta_{1}: \mathcal{P}^{+} \rightarrow \overline{\mathcal{P}}^{1}$ as follows. For $\lambda=\left(\lambda_{1}, \lambda_{2}, \cdots\right) \in \mathcal{P}^{+}$, let

$$
\theta_{1}(\lambda)=\left(\left\langle\lambda_{1}^{\prime}\right\rangle,\left\langle\lambda_{1}-1\right\rangle,\left\langle\lambda_{2}^{\prime}-1\right\rangle,\left\langle\lambda_{2}-2\right\rangle,\left\langle\lambda_{3}^{\prime}-2\right\rangle,\left\langle\lambda_{3}-3\right\rangle, \cdots\right) .
$$

It is easy to see that $\theta_{1}(\lambda) \in \overline{\mathcal{P}}^{1}$ and $\theta_{1}$ is a bijection. We have the following combinatorial lemma that is equivalent to [M, 1.7], where an elegant proof can be found.

Lemma 5.1. Let $i=1, \cdots, N$ with $N \geq \lambda_{1}^{\prime}$. The set $\left\{\lambda_{i}^{\prime}-i+1 \mid \lambda_{i}^{\prime}-i+1>\right.$ $0\} \cup\left\{-\lambda_{i}+i \mid \lambda_{i}-i<0\right\}$ is a permutation of the set $\{1,2, \cdots, N\}$.

For $a, b \in \overline{\mathcal{P}}^{1}$, we define

$$
(a \mid b)_{s}:=\sum_{r \in \frac{1}{2} \mathbb{N}}(-1)^{2 r} a_{r} b_{r} .
$$

For $\lambda \in \mathcal{P}^{+}$, we define

$$
\left(\lambda+2 \rho_{1} \mid \lambda\right)_{1}:=\sum_{i \in \mathbb{N}} \lambda_{i}\left(\lambda_{i}-2 i\right)
$$

Proposition 5.1. For $\lambda \in \mathcal{P}^{+}$, we have

$$
\left(\lambda+2 \rho_{1} \mid \lambda\right)_{1}=\left(\theta_{1}(\lambda) \mid \theta_{1}(\lambda)\right)_{s} .
$$


Proof. Let $s$ be the smallest positive integer such that $\lambda_{s}-s \leq 0$, and $l$ the length of $\lambda$. We have

$$
\begin{aligned}
\left(\theta_{1}(\lambda) \mid \theta_{1}(\lambda)\right)_{s} & =\sum_{i}\left\langle\lambda_{i}-i\right\rangle^{2}-\sum_{j}\left\langle\lambda_{j}^{\prime}-j+1\right\rangle^{2} \\
& =\sum_{i=1}^{l}\left(\lambda_{i}-i\right)\left(\lambda_{i}-i\right)-\sum_{j=s}^{l}\left(\lambda_{i}-i\right)^{2}-\sum_{j}\left\langle\lambda_{j}^{\prime}-j+1\right\rangle^{2} \\
& =\sum_{i=1}^{l}\left(\lambda_{i}-i\right)\left(\lambda_{i}-i\right)-\sum_{j=s}^{l}\left(-\lambda_{i}+i\right)^{2}-\sum_{j}\left\langle\lambda_{j}^{\prime}-j+1\right\rangle^{2} \\
& =\sum_{i=1}^{l}\left(\lambda_{i}-i\right)\left(\lambda_{i}-i\right)-\sum_{j=1}^{\lambda_{1}^{\prime}} j^{2} \text { by Lemma } \underline{5.1} \\
& =\sum_{i=1}^{l} \lambda_{i}\left(\lambda_{i}-2 i\right)+\sum_{i=1}^{l} i^{2}-\sum_{j=1}^{\lambda_{1}^{\prime}} j^{2} \\
& =\sum_{i=1}^{l} \lambda_{i}\left(\lambda_{i}-2 i\right)=\left(\lambda+2 \rho_{1} \mid \lambda\right)_{1} .
\end{aligned}
$$

We also need to consider a slightly different setup. Let $\overline{\mathcal{P}}^{2}$ denote the set of sequences $a=\left(a_{0}, a_{\frac{1}{2}}, a_{1}, a_{\frac{3}{2}}, \cdots\right)$, where $\left(a_{0}, a_{1}, a_{2}, \cdots\right)$ and $\left(a_{\frac{1}{2}}, a_{\frac{3}{2}}, a_{\frac{5}{2}}, \cdots\right)$ are strict partitions, such that $a_{s}=0$ implies $a_{s+\frac{1}{2}}=0$ for all $s$. Let $\lambda$ be a partition, whose rows and columns we index with $\mathbb{Z}_{+}$, i.e. $\lambda=\left(\lambda_{0}, \lambda_{1}, \cdots\right)$. We define similarly an element $\theta_{2}(\lambda) \in \overline{\mathcal{P}}^{2}$ by

$$
\theta_{2}(\lambda):=\left(\left\langle\lambda_{0}\right\rangle,\left\langle\lambda_{0}^{\prime}-1\right\rangle,\left\langle\lambda_{1}-1\right\rangle,\left\langle\lambda_{1}^{\prime}-2\right\rangle,\left\langle\lambda_{2}-2\right\rangle,\left\langle\lambda_{2}^{\prime}-3\right\rangle, \cdots\right) .
$$

For $a, b \in \overline{\mathcal{P}}^{2}$, we define

$$
(a \mid b)_{s}:=\sum_{r \in \frac{1}{2} \mathbb{Z}_{+}}(-1)^{2 r} a_{r} b_{r} .
$$

For $\lambda \in \mathcal{P}^{+}$, we define $\left(\lambda+2 \rho_{2} \mid \lambda\right)_{2}:=\sum_{i \in \mathbb{Z}_{+}} \lambda_{i}\left(\lambda_{i}-2 i\right)$. Similarly one can show the following.

Proposition 5.2. For $\lambda \in \mathcal{P}^{+}$, we have

$$
\left(\lambda+2 \rho_{2} \mid \lambda\right)_{2}=\left(\theta_{2}(\lambda) \mid \theta_{2}(\lambda)\right)_{s} .
$$

Corollary 5.1. Let $\lambda, \mu$ be partitions. For $i=1,2$, we have $\left(\lambda+2 \rho_{i} \mid \lambda\right)_{i}=\left(\mu+2 \rho_{i} \mid \mu\right)_{i}$ if and only if $\left(\theta_{i}(\lambda) \mid \theta_{i}(\lambda)\right)_{s}=\left(\theta_{i}(\mu) \mid \theta_{i}(\mu)\right)_{s}$. 
5.2. Formulas for the $\overline{\mathfrak{u}}_{-}$-homology groups. We keep the notation of Section 4. Suppose that $\mu=\sum_{i \in \mathbb{N}} \mu_{i} \epsilon_{i}-\sum_{j \in-\mathbb{Z}_{+}} \mu_{j} \epsilon_{j}+n \Lambda_{0}^{\mathfrak{x}} \in \mathcal{P}_{\mathfrak{l}}^{+}$is given, where we assume that $\mu_{j}=0$ for $j \leq 0$ if $\mathfrak{g} \neq \widehat{\mathfrak{g l}}_{\infty}$. By definition, $\mu^{+}=\left(\mu_{1}, \mu_{2}, \cdots\right)$ and $\mu^{-}=\left(\mu_{0}, \mu_{-1}, \mu_{-2}, \cdots\right)$ are partitions. Set $\theta_{1}\left(\mu^{+}\right)=\left(a_{\frac{1}{2}}, a_{1}, a_{\frac{3}{2}}, \cdots\right) \in \overline{\mathcal{P}}^{1}$ and $\theta_{2}\left(\mu^{-}\right)=\left(b_{0}, b_{\frac{1}{2}}, b_{1}, b_{\frac{3}{2}}, \cdots\right) \in \overline{\mathcal{P}}^{2}$. We define

$$
\theta(\mu):= \begin{cases}\sum_{r \in \frac{1}{2} \mathbb{N}} a_{r} \delta_{r}-\sum_{s \in \frac{1}{2} \mathbb{Z}_{+}} b_{s} \delta_{-s}+\bar{n} \bar{\Lambda}_{0}^{\mathfrak{a}}, & \text { if } \mathfrak{g}=\widehat{\mathfrak{g l}}_{\infty}, \\ \sum_{r \in \frac{1}{2} \mathbb{N}} a_{r} \delta_{r}+\bar{n} \bar{\Lambda}_{0}^{\mathfrak{x}}, & \text { if } \mathfrak{g}=\mathfrak{b}_{\infty}, \mathfrak{c}_{\infty}, \mathfrak{d}_{\infty},\end{cases}
$$

where $\bar{n}=n\left\langle\Lambda_{0}^{\mathfrak{x}}, K\right\rangle$. It is not difficult to see that $\theta(\mu) \in \mathcal{P}_{\overline{\mathfrak{l}}}^{+}$and $\theta$ yields a bijection from $\mathcal{P}_{\mathfrak{l}}^{+}$to $\mathcal{P}_{\overline{\mathfrak{l}}}^{+}$. In particular, $\theta\left(\Lambda^{\mathfrak{x}}(\lambda)\right)=\bar{\Lambda}^{\mathfrak{x}}(\lambda)$ for $\lambda \in \mathcal{P}(G)$.

For $\lambda \in \mathcal{P}(G)$, we put

$$
\mathrm{L}\left(\mathfrak{g}, \Lambda^{\mathfrak{x}}(\lambda)\right):= \begin{cases}L\left(\mathfrak{g}, \Lambda^{\mathfrak{d}}(\lambda)\right) \oplus L\left(\mathfrak{g}, \Lambda^{\mathfrak{d}}(\tilde{\lambda})\right), & \text { if } G=\mathrm{O}(2 \ell), \\ L\left(\mathfrak{g}, \Lambda^{\mathfrak{x}}(\lambda)\right), & \text { otherwise. }\end{cases}
$$

We define $\mathrm{L}\left(\overline{\mathfrak{g}}, \bar{\Lambda}^{\mathfrak{x}}(\lambda)\right)$ in a similar way. For $w \in W^{0}$ we put

$$
\mathrm{L}\left(\overline{\mathfrak{l}}, \theta\left(w \circ \Lambda^{\mathfrak{x}}(\lambda)\right)\right):= \begin{cases}L\left(\overline{\mathfrak{l}}, \theta\left(w \circ \Lambda^{\mathfrak{d}}(\lambda)\right)\right) \oplus L\left(\overline{\mathfrak{l}}, \theta\left(w \circ \Lambda^{\mathfrak{d}}(\tilde{\lambda})\right)\right), & \text { if } G=\mathrm{O}(2 \ell), \\ L\left(\overline{\mathfrak{l}}, \theta\left(w \circ \Lambda^{\mathfrak{x}}(\lambda)\right)\right), & \text { otherwise }\end{cases}
$$

Also $L\left(\mathfrak{l}, w \circ \Lambda^{\mathfrak{x}}(\lambda)\right)$ is defined similarly.

Note that $\operatorname{chL}\left(\overline{\mathfrak{g}}, \bar{\Lambda}^{\mathfrak{x}}(\lambda)\right)=\omega^{\mathfrak{x}}\left(\operatorname{chL}\left(\mathfrak{g}, \Lambda^{\mathfrak{x}}(\lambda)\right)\right)$ and $\operatorname{ch} L(\overline{\mathfrak{l}}, \theta(\eta))=\omega^{\mathfrak{x}}(\operatorname{ch} L(\mathfrak{l}, \eta))$, for $\eta \in \mathcal{P}_{\mathfrak{l}}^{+}$.

Lemma 5.2. Let $\lambda \in \mathcal{P}(G)$ and $\bar{\mu} \in \mathcal{P}_{\overline{\mathfrak{l}}}^{+}$. If $L(\overline{\mathfrak{l}}, \bar{\mu})$ appears in $\Lambda^{k} \overline{\mathfrak{u}}_{-} \otimes \mathrm{L}\left(\overline{\mathfrak{g}}, \bar{\Lambda}^{\mathfrak{x}}(\lambda)\right)$, then there exists a unique $\mu \in \mathcal{P}_{\mathfrak{l}}^{+}$, with $\theta(\mu)=\bar{\mu}$, such that $L(\mathfrak{l}, \mu)$ appears in $\Lambda^{k} \mathfrak{u}_{-} \otimes \mathrm{L}\left(\mathfrak{g}, \Lambda^{\mathfrak{x}}(\lambda)\right)$ with the same multiplicity.

Proof. Since we have $\omega^{\mathfrak{x}}\left(\operatorname{chL}\left(\mathfrak{g}, \Lambda^{\mathfrak{x}}(\lambda)\right)\right)=\operatorname{chL}\left(\overline{\mathfrak{g}}, \bar{\Lambda}^{\mathfrak{x}}(\lambda)\right)$ for $\lambda \in \mathcal{P}(G)$, we conclude that

$$
\omega^{\mathfrak{x}}\left(\operatorname{ch}\left[\Lambda^{k} \mathfrak{u}_{-} \otimes \mathrm{L}\left(\mathfrak{g}, \Lambda^{\mathfrak{x}}(\lambda)\right)\right]\right)=\operatorname{ch}\left[\Lambda^{k} \overline{\mathfrak{u}}_{-} \otimes \mathrm{L}\left(\overline{\mathfrak{g}}, \bar{\Lambda}^{\mathfrak{x}}(\lambda)\right)\right] .
$$

Since $\theta$ is a bijection, there exists a unique $\mu \in \mathcal{P}_{\mathfrak{l}}^{+}$such that $\theta(\mu)=\bar{\mu}$. Therefore $L(\mathfrak{l}, \mu)$ is a composition factor of $\Lambda^{k} \mathfrak{u}_{-} \otimes \mathrm{L}\left(\mathfrak{g}, \Lambda^{\mathfrak{x}}(\lambda)\right)$ if and only if $L(\overline{\mathfrak{l}}, \theta(\mu))$ is a composition factor of $\Lambda^{k} \overline{\mathfrak{u}}_{-} \otimes \mathrm{L}\left(\overline{\mathfrak{g}}, \bar{\Lambda}^{\mathfrak{x}}(\lambda)\right)$. Furthermore they have the same multiplicity.

The following lemma is crucial in the sequel.

Lemma 5.3. For $\mu \in \mathcal{P}_{\mathfrak{l}}^{+}$, we have

$$
\left(\mu+2 \rho_{c} \mid \mu\right)_{c}=\left(\theta(\mu)+2 \rho_{s} \mid \theta(\mu)\right)_{s} .
$$

In particular, for $\lambda \in \mathcal{P}(G)$, we have $\left(\Lambda^{\mathfrak{x}}(\lambda)+2 \rho_{c} \mid \Lambda^{\mathfrak{x}}(\lambda)\right)_{c}=\left(\bar{\Lambda}^{\mathfrak{x}}(\lambda)+2 \rho_{s} \mid \bar{\Lambda}^{\mathfrak{x}}(\lambda)\right)_{s}$. 
Proof. Case 1. $\mathfrak{g}=\widehat{\mathfrak{g l}}_{\infty}$. Suppose that $\mu=\sum_{i \in \mathbb{N}} \mu_{i} \epsilon_{i}-\sum_{i \in-\mathbb{Z}_{+}} \mu_{i} \epsilon_{i}+n \Lambda_{0}^{\mathfrak{a}}$, where $\mu^{+}=\left(\mu_{1}, \mu_{2}, \ldots\right)$ and $\mu^{-}=\left(\mu_{0}, \mu_{-1}, \ldots\right)$ are partitions. Let $\theta_{1}\left(\mu^{+}\right)=\left(a_{1}, a_{\frac{3}{2}}, \ldots\right)$ and $\theta_{2}\left(\mu^{-}\right)=\left(b_{0}, b_{\frac{1}{2}}, \ldots\right)$. Using Proposition 5.1 and Proposition 5.2, we compute:

$$
\begin{aligned}
& \begin{aligned}
& \left.\mu+2 \rho_{c} \mid \mu\right)_{c} \\
= & \left(\sum_{i \in \mathbb{N}} \mu_{i} \epsilon_{i}+2 \rho_{c} \mid \sum_{i \in \mathbb{N}} \mu_{i} \epsilon_{i}\right)_{c}+\left(\sum_{i \in-\mathbb{Z}_{+}} \mu_{i} \epsilon_{i}-2 \rho_{c} \mid \sum_{i \in-\mathbb{Z}_{+}} \mu_{i} \epsilon_{i}\right)_{c} \\
& +2 n\left(\Lambda_{0}^{\mathfrak{a}} \mid \sum_{i \in \mathbb{N}} \mu_{i} \epsilon_{i}\right)_{c}-2 n\left(\Lambda_{0}^{\mathfrak{a}} \mid \sum_{i \in-\mathbb{Z}_{+}} \mu_{i} \epsilon_{i}\right)_{c}
\end{aligned} \\
& =\sum_{i \in \mathbb{N}} \mu_{i}\left(\mu_{i}-2 i\right)+\sum_{i \in \mathbb{Z}_{+}} \mu_{-i}\left(\mu_{-i}-2 i\right)-n\left(\sum_{i \in \mathbb{N}} \mu_{i}+\sum_{i \in-\mathbb{Z}_{+}} \mu_{i}\right) \\
& =\left(\mu^{+}+2 \rho_{1} \mid \mu^{+}\right)_{1}+\left(\mu^{-}+2 \rho_{2} \mid \mu^{-}\right)_{2}-n\left(\sum_{i \in \mathbb{N}} \mu_{i}+\sum_{i \in-\mathbb{Z}_{+}} \mu_{i}\right) \\
& =\left(\theta_{1}\left(\mu^{+}\right) \mid \theta_{1}\left(\mu^{+}\right)\right)_{s}+\left(\theta_{2}\left(\mu^{-}\right) \mid \theta_{2}\left(\mu^{-}\right)\right)_{s}-n\left(\sum_{r \in \frac{1}{2} \mathbb{N}} a_{r}+\sum_{r \in \frac{1}{2} \mathbb{Z}_{+}} b_{r}\right) \\
& =\left(\sum_{r \in \frac{1}{2} \mathbb{N}} a_{r} \delta_{r} \mid \sum_{r \in \frac{1}{2} \mathbb{N}} a_{r} \delta_{r}\right)_{s}+\left(\sum_{r \in \frac{1}{2} \mathbb{Z}_{+}} b_{r} \delta_{r} \mid \sum_{r \in \frac{1}{2} \mathbb{Z}_{+}} b_{r} \delta_{r}\right)_{s} \\
& \quad+2 n\left(\bar{\Lambda}_{0}^{\mathfrak{a}} \mid \sum_{r \in \frac{1}{2} \mathbb{N}} a_{r} \delta_{r}\right)-2 n\left(\bar{\Lambda}_{0}^{\mathfrak{a}} \mid \sum_{r \in \frac{1}{2} \mathbb{Z}_{+}} b_{r} \delta_{r}\right)_{s} \\
& =(\theta(\mu) \mid \theta(\mu))_{s}=\left(\theta(\mu)+2 \rho_{s} \mid \theta(\mu)\right)_{s},
\end{aligned}
$$

where $\rho_{s}=0$ in this case.

Next, we assume that $\mathfrak{g}=\mathfrak{x}_{\infty}$ with $\mathfrak{x} \in\{\mathfrak{b}, \mathfrak{c}, \mathfrak{d}\}$. Suppose that $\mu=\sum_{i \in \mathbb{N}} \mu_{i} \epsilon_{i}+n \Lambda_{0}^{\mathfrak{x}}$, where $\mu^{\circ}=\left(\mu_{1}, \mu_{2}, \ldots\right)$ is a partition. Let $\theta_{1}\left(\mu^{\circ}\right)=\left(a_{1}, a_{\frac{3}{2}}, \ldots\right)$.

Case 2. $\mathfrak{g}=\mathfrak{b}_{\infty}$.

$$
\begin{aligned}
& \left(\mu+2 \rho_{c} \mid \mu\right)_{c} \\
& =\left(\sum_{i \in \mathbb{N}} \mu_{i} \epsilon_{i}+2 \rho_{c} \mid \sum_{i \in \mathbb{N}} \mu_{i} \epsilon_{i}\right)_{c}+2 n\left(\Lambda_{0}^{\mathfrak{b}} \mid \sum_{i \in \mathbb{N}} \mu_{i} \epsilon_{i}\right)_{c}=\sum_{i \in \mathbb{N}} \mu_{i}\left(\mu_{i}-2 i+1\right)-n \sum_{i \in \mathbb{N}} \mu_{i} \\
& =\left(\mu^{\circ}+\rho_{1} \mid \mu^{\circ}\right)_{1}+\sum_{i \in \mathbb{N}} \mu_{i}-n \sum_{i \in \mathbb{N}} \mu_{i}=\left(\theta_{1}\left(\mu^{\circ}\right) \mid \theta_{1}\left(\mu^{\circ}\right)\right)_{s}+\sum_{r \in \frac{1}{2} \mathbb{N}} a_{r}-n \sum_{r \in \frac{1}{2} \mathbb{N}} a_{r} \\
& =\left(\sum_{r \in \frac{1}{2} \mathbb{N}} a_{r} \delta_{r} \mid \sum_{r \in \frac{1}{2} \mathbb{N}} a_{r} \delta_{r}\right)_{s}+2\left(\rho_{s} \mid \sum_{r \in \frac{1}{2} \mathbb{N}} a_{r} \delta_{r}\right)_{s}+n\left(\bar{\Lambda}_{0}^{\mathfrak{b}} \mid \sum_{r \in \frac{1}{2} \mathbb{N}} a_{r} \delta_{r}\right)_{s} \\
& =\left(\sum_{r \in \frac{1}{2} \mathbb{N}} a_{r} \delta_{r}+\frac{1}{2} n \bar{\Lambda}_{0}^{\mathfrak{b}}+2 \rho_{s} \mid \sum_{r \in \frac{1}{2} \mathbb{N}} a_{r} \delta_{r}+\frac{1}{2} n \bar{\Lambda}_{0}^{\mathfrak{b}}\right)_{s}=\left(\theta(\mu)+2 \rho_{s} \mid \theta(\mu)\right)_{s} .
\end{aligned}
$$


Case 3. $\mathfrak{g}=\mathfrak{c}_{\infty}$.

$$
\begin{aligned}
& \left(\mu+2 \rho_{c} \mid \mu\right)_{c} \\
& =\left(\sum_{i \in \mathbb{N}} \mu_{i} \epsilon_{i}+2 \rho_{c} \mid \sum_{i \in \mathbb{N}} \mu_{i} \epsilon_{i}\right)_{c}+2 n\left(\Lambda_{0}^{\mathfrak{c}} \mid \sum_{i \in \mathbb{N}} \mu_{i} \epsilon_{i}\right)_{c}=\sum_{i \in \mathbb{N}} \mu_{i}\left(\mu_{i}-2 i\right)-2 n \sum_{i \in \mathbb{N}} \mu_{i} \\
& =\left(\mu^{\circ}+\rho_{1} \mid \mu^{\circ}\right)_{1}-2 n \sum_{i \in \mathbb{N}} \mu_{i}=\left(\theta_{1}\left(\mu^{\circ}\right) \mid \theta_{1}\left(\mu^{\circ}\right)\right)_{s}-2 n \sum_{r \in \frac{1}{2} \mathbb{N}} a_{r} \\
& =\left(\sum_{r \in \frac{1}{2} \mathbb{N}} a_{r} \delta_{r} \mid \sum_{r \in \frac{1}{2} \mathbb{N}} a_{r} \delta_{r}\right)_{s}+2 n\left(\bar{\Lambda}_{0}^{\mathfrak{c}} \mid \sum_{r \in \frac{1}{2} \mathbb{N}} a_{r} \delta_{r}\right)_{s} \\
& =\left(\sum_{r \in \frac{1}{2} \mathbb{N}} a_{r} \delta_{r}+n \bar{\Lambda}_{0}^{\mathfrak{c}}+2 \rho_{s} \mid \sum_{r \in \frac{1}{2} \mathbb{N}} a_{r} \delta_{r}+n \bar{\Lambda}_{0}^{\mathfrak{c}}\right)_{s}=\left(\theta(\mu)+2 \rho_{s} \mid \theta(\mu)\right)_{s} .
\end{aligned}
$$

Note that $\rho_{s}=0$ in this case.

Case $4 \cdot \mathfrak{g}=\mathfrak{d}_{\infty}$.

$$
\begin{aligned}
& \left(\mu+2 \rho_{c} \mid \mu\right)_{c} \\
& =\left(\sum_{i \in \mathbb{N}} \mu_{i} \epsilon_{i}+2 \rho_{c} \mid \sum_{i \in \mathbb{N}} \mu_{i} \epsilon_{i}\right)_{c}+2 n\left(\Lambda_{0}^{\mathfrak{d}} \mid \sum_{i \in \mathbb{N}} \mu_{i} \epsilon_{i}\right)_{c}=\sum_{i \in \mathbb{N}} \mu_{i}\left(\mu_{i}-2(i-1)\right)-n \sum_{i \in \mathbb{N}} \mu_{i} \\
& =\left(\mu^{\circ}+\rho_{1} \mid \mu^{\circ}\right)_{1}+2 \sum_{i \in \mathbb{N}} \mu_{i}-n \sum_{i \in \mathbb{N}} \mu_{i}=\left(\theta_{1}\left(\mu^{\circ}\right) \mid \theta_{1}\left(\mu^{\circ}\right)\right)_{s}+2 \sum_{r \in \frac{1}{2} \mathbb{N}} a_{r}-n \sum_{r \in \frac{1}{2} \mathbb{N}} a_{r} \\
& =\left(\sum_{r \in \frac{1}{2} \mathbb{N}} a_{r} \delta_{r} \mid \sum_{r \in \frac{1}{2} \mathbb{N}} a_{r} \delta_{r}\right)_{s}+2\left(\rho_{s} \mid \sum_{r \in \frac{1}{2} \mathbb{N}} a_{r} \delta_{r}\right)_{s}+n\left(\bar{\Lambda}_{0}^{\mathfrak{b}} \mid \sum_{r \in \frac{1}{2} \mathbb{N}} a_{r} \delta_{r}\right)_{s} \\
& =\left(\sum_{r \in \frac{1}{2} \mathbb{N}} a_{r} \delta_{r}+\frac{1}{2} n \bar{\Lambda}_{0}^{\mathfrak{d}}+2 \rho_{s} \mid \sum_{r \in \frac{1}{2} \mathbb{N}} a_{r} \delta_{r}+\frac{1}{2} n \bar{\Lambda}_{0}^{\mathfrak{d}}\right)_{s}=\left(\theta(\mu)+2 \rho_{s} \mid \theta(\mu)\right)_{s} .
\end{aligned}
$$

Remark 5.1. Note that for $\lambda \in \mathcal{P}(\mathrm{O}(2 \ell))$

$$
\left(\Lambda^{\mathfrak{d}}(\lambda)+2 \rho_{c} \mid \Lambda^{\mathfrak{d}}(\lambda)\right)_{c}=\left(\Lambda^{\mathfrak{d}}(\tilde{\lambda})+2 \rho_{c} \mid \Lambda^{\mathfrak{d}}(\tilde{\lambda})\right)_{c} .
$$

Corollary 5.2. Let $\lambda \in \mathcal{P}(G)$ and let $\Lambda^{k}\left(\overline{\mathfrak{u}}_{-}\right) \otimes \mathrm{L}\left(\overline{\mathfrak{g}}, \bar{\Lambda}^{\mathfrak{x}}(\lambda)\right)=\bigoplus_{\eta \in \mathcal{P}_{\overline{\mathfrak{I}}}^{+}} L(\overline{\mathfrak{l}}, \eta)^{m_{\eta}}$ be its decomposition into irreducible $\overline{\mathfrak{l}}$-modules. Then $\eta=\theta\left(w \circ \Lambda^{\mathfrak{x}}(\lambda)\right)$, for some $w \in W_{k}^{0}$, if and only if $\left(\eta+2 \rho_{s} \mid \eta\right)_{s}=\left(\bar{\Lambda}^{\mathfrak{x}}(\lambda)+2 \rho_{s} \mid \bar{\Lambda}^{\mathfrak{x}}(\lambda)\right)_{s}$.

Proof. Suppose that there exits $w \in W_{k}^{0}$ with $\eta=\theta\left(w \circ \Lambda^{\mathfrak{x}}(\lambda)\right)$. Then by Lemma 5.3 and the $W$-invariance of $(\cdot \mid \cdot)_{c}$ we have

$\left(\eta+2 \rho_{s} \mid \eta\right)_{s}=\left(w \circ \Lambda^{\mathfrak{x}}(\lambda)+2 \rho_{c} \mid w \circ \Lambda^{\mathfrak{x}}(\lambda)\right)_{c}=\left(\Lambda^{\mathfrak{x}}(\lambda)+2 \rho_{c} \mid \Lambda^{\mathfrak{x}}(\lambda)\right)_{c}=\left(\bar{\Lambda}^{\mathfrak{x}}(\lambda)+2 \rho_{s} \mid \bar{\Lambda}^{\mathfrak{x}}(\lambda)\right)_{s}$. 
Conversely, suppose that $\left(\eta+2 \rho_{s} \mid \eta\right)_{s}=\left(\bar{\Lambda}^{\mathfrak{x}}(\lambda)+2 \rho_{s} \mid \bar{\Lambda}^{\mathfrak{x}}(\lambda)\right)_{s}$. By Lemma 5.3 we have

$$
\left(\theta^{-1}(\eta)+2 \rho_{c} \mid \theta^{-1}(\eta)\right)_{c}=\left(\Lambda^{\mathfrak{x}}(\lambda)+2 \rho_{c} \mid \Lambda^{\mathfrak{x}}(\lambda)\right)_{c} .
$$

By Proposition 4.2 (i) there exist $w \in W_{k}^{0}$ such that $\theta^{-1}(\eta)=w \circ \Lambda^{\mathfrak{x}}(\lambda)$.

Remark 5.2. The multiplicity $m_{\eta}$ equals 1 if $\eta$ satisfies the condition of Corollary 5.2 for $\mathfrak{x}=\mathfrak{a}, \mathfrak{b}, \mathfrak{c}$ or $\mathfrak{x}=\mathfrak{d}$ with $\lambda \neq \tilde{\lambda}$. In the case of $\mathfrak{x}=\mathfrak{d}$ with $\lambda=\tilde{\lambda}$ we have $m_{\eta}=2$. This follows from (5.2), Proposition 4.2 and Lemma 5.2.

Theorem 5.1. Let $\lambda \in \mathcal{P}(G), k \in \mathbb{Z}_{+}$, and $\theta$ as in (5.1). As $\overline{\mathfrak{l}}$-modules we have

$$
\mathrm{H}_{k}\left(\overline{\mathfrak{u}}_{-} ; \mathrm{L}\left(\overline{\mathfrak{g}}, \bar{\Lambda}^{\mathfrak{x}}(\lambda)\right)\right) \cong \bigoplus_{w \in W_{k}^{0}} \mathrm{~L}\left(\overline{\mathfrak{l}}, \theta\left(w \circ \Lambda^{\mathfrak{x}}(\lambda)\right)\right) .
$$

In particular, $\operatorname{ch}\left[\mathrm{H}_{k}\left(\overline{\mathfrak{u}}_{-} ; \mathrm{L}\left(\overline{\mathfrak{g}}, \bar{\Lambda}^{\mathfrak{x}}(\lambda)\right)\right)\right]=\omega^{\mathfrak{x}}\left(\operatorname{ch}\left[\mathrm{H}_{k}\left(\mathfrak{u}_{-} ; \mathrm{L}\left(\mathfrak{g}, \Lambda^{\mathfrak{x}}(\lambda)\right)\right)\right]\right)$.

Proof. Let $\mu \in \mathcal{P}_{\mathfrak{l}}^{+}$be such that $L(\mathfrak{l}, \mu)$ is a composition factor of $\mathrm{H}_{k}\left(\mathfrak{u}_{-} ; \mathrm{L}\left(\mathfrak{g}, \Lambda^{\mathfrak{x}}(\lambda)\right)\right)$. Then it is precisely a composition factor of $\Lambda^{k} \mathfrak{u}_{-} \otimes \mathrm{L}\left(\mathfrak{g}, \Lambda^{\mathfrak{x}}(\lambda)\right)$ with $\left(\mu+2 \rho_{c} \mid \mu\right)_{c}=$ $\left(\Lambda^{\mathfrak{x}}(\lambda)+2 \rho_{c} \mid \Lambda^{\mathfrak{x}}(\lambda)\right)_{c}$ by Proposition 4.2. Furthermore each appears with multiplicity given in Remark [5.2. By Corollary 5.2 the corresponding $\theta(\mu)$ 's are precisely the weights in $\mathcal{P}_{\overline{\mathfrak{l}}}^{+}$such that $L(\overline{\mathfrak{l}}, \theta(\mu))$ appears as a composition factor of $\Lambda^{k} \overline{\mathfrak{u}}_{-} \otimes \mathrm{L}\left(\overline{\mathfrak{g}}, \bar{\Lambda}^{\mathfrak{x}}(\lambda)\right)$ with $\left(\theta(\mu)+2 \rho_{s} \mid \theta(\mu)\right)_{s}=\left(\bar{\Lambda}^{\mathfrak{x}}(\lambda)+2 \rho_{s} \mid \bar{\Lambda}^{\mathfrak{x}}(\lambda)\right)_{s}$, which has the same multiplicity.

Now, Theorems 3.3 3.6, and the Euler-Poincaré principle imply

$$
\sum_{k=0}^{\infty}(-1)^{k} \operatorname{ch}\left[\mathrm{H}_{k}\left(\overline{\mathfrak{u}}_{-} ; \mathrm{L}\left(\overline{\mathfrak{g}}, \bar{\Lambda}^{\mathfrak{x}}(\lambda)\right)\right)\right]=\sum_{k=0}^{\infty} \sum_{w \in W_{k}^{0}}(-1)^{k} \mathcal{H}_{\lambda, w},
$$

where

$$
\mathcal{H}_{\lambda, w}= \begin{cases}H S_{\left(\lambda_{w}^{+}\right)^{\prime}}\left(\mathbf{x}_{>0}\right) H S_{\lambda_{w}^{-}}\left(\mathbf{x}_{\leq 0}^{-1}\right), & \text { if } G=\mathrm{GL}(\ell), \\ H S_{\left(\lambda_{w}\right)^{\prime}}\left(\mathbf{x}_{>0}\right), & \text { if } G=\operatorname{Pin}(2 \ell), \operatorname{Sp}(2 \ell), \mathrm{O}(2 \ell+1), \\ H S_{\left(\lambda_{w}\right)^{\prime}}\left(\mathbf{x}_{>0}\right)+H S_{\left(\tilde{\lambda}_{w}\right)^{\prime}}\left(\mathbf{x}_{>0}\right), & \text { if } G=\mathrm{O}(2 \ell) .\end{cases}
$$

Since all the highest weights are distinct, we conclude from Proposition 4.3 that

$$
\operatorname{ch}\left[\mathrm{H}_{k}\left(\overline{\mathfrak{u}}_{-} ; \mathrm{L}\left(\overline{\mathfrak{g}}, \bar{\Lambda}^{\mathfrak{x}}(\lambda)\right)\right)\right]=\sum_{w \in W_{k}^{0}} \mathcal{H}_{\lambda, w} .
$$

The right hand side is equal to $\omega^{\mathfrak{x}}\left(\operatorname{ch}\left[\mathrm{H}_{k}\left(\mathfrak{u}_{-} ; \mathrm{L}\left(\mathfrak{g}, \Lambda^{\mathfrak{x}}(\lambda)\right)\right)\right]\right)$ by Proposition 2.5.

Theorem [5.1] and Corollary [5.2 imply the following super analogue of Proposition 4.2 (ii), which is the converse of Proposition of 4.3 . 
Corollary 5.3. If $L(\overline{\mathfrak{l}}, \eta)$ is an irreducible $\overline{\mathfrak{l}}$-module in $\Lambda^{k}\left(\overline{\mathfrak{u}}_{-}\right) \otimes \mathrm{L}\left(\overline{\mathfrak{g}}, \bar{\Lambda}^{\mathfrak{x}}(\lambda)\right)$ such that $\left(\eta+2 \rho_{s} \mid \eta\right)_{s}=\left(\bar{\Lambda}^{\mathfrak{x}}(\lambda)+2 \rho_{s} \mid \bar{\Lambda}^{\mathfrak{x}}(\lambda)\right)_{s}$, then $L(\overline{\mathfrak{l}}, \eta)$ is a component of $\mathrm{H}_{k}\left(\overline{\mathfrak{u}}_{-} ; \mathrm{L}\left(\overline{\mathfrak{g}}, \bar{\Lambda}^{\mathfrak{x}}(\lambda)\right)\right)$. Furthermore the multiplicities coincide.

Remark 5.3. In the spirit of [CWZ] we can use Serganova's homological interpretation of Kazhdan-Lusztig polynomials [Se] to reformulate Theorem 5.1 into a statement about equality of certain Kazhdan-Lusztig polynomials for $\mathfrak{g}$ and $\overline{\mathfrak{g}}$.

\section{Resolution in terms of Generalized Verma modules}

Let $V$ be a $\overline{\mathfrak{g}}$-module, on which the action of $\overline{\mathfrak{u}}_{+}$is locally nilpotent. For $c \in \mathbb{C}$, we define

$$
V^{c}:=\left\{v \in V \mid(\bar{\Omega}-c)^{n} v=0, n \gg 0\right\},
$$

i.e. $V^{c}$ is the generalized $\bar{\Omega}$-eigenspace corresponding to the eigenvalue $c$. Clearly we have $V=\bigoplus_{c \in \mathbb{C}} V^{c}$.

We can now construct a resolution of generalized Verma modules for $\mathrm{L}\left(\overline{\mathfrak{g}}, \bar{\Lambda}^{\mathfrak{x}}(\lambda)\right)$.

Note that $\overline{\mathfrak{g}} / \overline{\mathfrak{p}}$ is a $\overline{\mathfrak{p}}$-module, on which $\overline{\mathfrak{u}}_{+}$acts trivially, and hence so is $\Lambda^{k}(\overline{\mathfrak{g}} / \overline{\mathfrak{p}})$, for all $k \in \mathbb{Z}_{+}$. Thus the induced module $U(\overline{\mathfrak{g}}) \otimes_{\overline{\mathfrak{p}}} \Lambda^{k}(\overline{\mathfrak{g}} / \overline{\mathfrak{p}})$ is a $\overline{\mathfrak{g}}$-module. For $\lambda \in \mathcal{P}(G)$ we consider the $\overline{\mathfrak{g}}$-module $Y_{k}=\left(U(\overline{\mathfrak{g}}) \otimes_{U(\overline{\mathfrak{p}})} \Lambda^{k}(\overline{\mathfrak{g}} / \overline{\mathfrak{p}})\right) \otimes \mathrm{L}\left(\overline{\mathfrak{g}}, \bar{\Lambda}^{\mathfrak{x}}(\lambda)\right)$. Let

$$
\ldots \stackrel{d_{k+1}}{\longrightarrow} Y_{k} \stackrel{d_{k}}{\longrightarrow} Y_{k-1} \stackrel{d_{k-1}}{\longrightarrow} \ldots \stackrel{d_{1}}{\longrightarrow} Y_{0} \stackrel{d_{0}}{\longrightarrow} \mathrm{L}\left(\overline{\mathfrak{g}}, \bar{\Lambda}^{\mathfrak{x}}(\lambda)\right) \rightarrow 0
$$

be the standard resolution of $\mathrm{L}\left(\overline{\mathfrak{g}}, \bar{\Lambda}^{\mathfrak{x}}(\lambda)\right)$ as in [KK] (cf. [GL, J, L] $)$. Put

$$
c_{\lambda}=\left(\bar{\Lambda}^{\mathfrak{x}}(\lambda)+2 \rho_{s} \mid \bar{\Lambda}^{\mathfrak{x}}(\lambda)\right)_{s} .
$$

The restriction of (6.1) to the generalized $c_{\lambda}$-eigenspace of $\bar{\Omega}$ produces a resolution of $\overline{\mathfrak{g}}$-modules

$$
\cdots \stackrel{d_{k+1}}{\longrightarrow} Y_{k}^{c_{\lambda}} \stackrel{d_{k}}{\longrightarrow} Y_{k-1}^{c_{\lambda}} \stackrel{d_{k-1}}{\longrightarrow} \cdots \stackrel{d_{1}}{\longrightarrow} Y_{0}^{c_{\lambda}} \stackrel{d_{0}}{\longrightarrow} \mathrm{L}\left(\overline{\mathfrak{g}}, \bar{\Lambda}^{\mathfrak{x}}(\lambda)\right) \rightarrow 0 .
$$

For $\nu \in \mathcal{P}_{\overline{\mathfrak{l}}}^{+}$, let $V(\nu)=U(\overline{\mathfrak{g}}) \otimes_{U(\overline{\mathfrak{p}})} L(\overline{\mathfrak{l}}, \nu)$ be the generalized Verma module, where $\overline{\mathfrak{u}}_{+}$acts trivially on $L(\overline{\mathfrak{l}}, \nu)$. We have the following counterpart of Theorem 8.7 of GL.

Theorem 6.1. For $\lambda \in \mathcal{P}(G)$, the (6.2) is a resolution of $\mathrm{L}\left(\overline{\mathfrak{g}}, \bar{\Lambda}^{\mathfrak{x}}(\lambda)\right)$ such that each $Y_{k}^{c_{\lambda}}$ has a flag of generalized Verma modules. Furthermore as $\overline{\mathfrak{l}}+\overline{\mathfrak{u}}_{-}$-modules we have

$$
Y_{k}^{c_{\lambda}} \cong \begin{cases}\bigoplus_{w \in W_{k}^{0}} V\left(\theta\left(w \circ \Lambda^{\mathfrak{x}}(\lambda)\right)\right), & \text { if } G \neq \mathrm{O}(2 \ell) \\ \bigoplus_{w \in W_{k}^{0}}\left[V\left(\theta\left(w \circ \Lambda^{\mathfrak{d}}(\lambda)\right)\right) \oplus V\left(\theta\left(w \circ \Lambda^{\mathfrak{D}}(\tilde{\lambda})\right)\right)\right], & \text { if } G=\mathrm{O}(2 \ell)\end{cases}
$$


Proof. To keep notation simple let us assume that $\mathfrak{g} \neq \mathfrak{d}_{\infty}$.

First we claim that $Y_{k} \cong U(\overline{\mathfrak{g}}) \otimes_{U(\overline{\mathfrak{p}})}\left(\Lambda^{k}(\overline{\mathfrak{g}} / \overline{\mathfrak{p}}) \otimes L\left(\overline{\mathfrak{g}}, \bar{\Lambda}^{\mathfrak{x}}(\lambda)\right)\right)$ has a flag of generalized Verma modules. This can be seen as follows. From the proof of Lemma 4.2 we see that the space $\Lambda^{k}(\overline{\mathfrak{g}} / \overline{\mathfrak{p}})$ decomposes into a finite direct sum of $\overline{\mathfrak{p}}$-modules, which is semisimple over $\overline{\mathfrak{l}}$ and on which $\overline{\mathfrak{u}}_{+}$acts trivially. On the other hand $L\left(\overline{\mathfrak{g}}, \bar{\Lambda}^{\mathfrak{x}}(\lambda)\right)$ has a $\overline{\mathfrak{p}}$-submodule, which as an $\overline{\mathfrak{l}}$-module is isomorphic to $L\left(\overline{\mathfrak{l}}, \bar{\Lambda}^{\mathfrak{x}}(\lambda)\right)$ and on which $\overline{\mathfrak{u}}_{+}$acts trivially. This module is precisely the $\overline{\mathfrak{u}}_{+}$-invariants of $L\left(\overline{\mathfrak{g}}, \bar{\Lambda}^{\mathfrak{x}}(\lambda)\right)$. Now we consider the $\overline{\mathfrak{u}}_{+}$-invariants of $L\left(\overline{\mathfrak{g}}, \bar{\Lambda}^{\mathfrak{x}}(\lambda)\right) / L\left(\overline{\mathfrak{l}}, \bar{\Lambda}^{\mathfrak{x}}(\lambda)\right)$, which is a direct sum of irreducible $\overline{\mathfrak{l}}$-modules. Continuing this way we see that $L\left(\overline{\mathfrak{g}}, \bar{\Lambda}^{\mathfrak{x}}(\lambda)\right)$ as a $\overline{\mathfrak{p}}$-module has an infinite filtration of $\overline{\mathfrak{p}}$-modules of the form

$$
L_{0}=L\left(\overline{\mathfrak{g}}, \bar{\Lambda}^{\mathfrak{x}}(\lambda)\right) \supseteq L_{1} \supseteq L_{2} \supseteq \cdots
$$

such that $L_{i} / L_{i+1}$ is a direct sum of irreducible $\overline{\mathfrak{l}}$-modules on which $\overline{\mathfrak{u}}_{+}$acts trivially. Thus the same is true for $\Lambda^{k}(\overline{\mathfrak{g}} / \overline{\mathfrak{p}}) \otimes L\left(\overline{\mathfrak{g}}, \bar{\Lambda}^{\mathfrak{x}}(\lambda)\right)$. Now $Y_{k}$ is the induced module and hence the claim follows. Since $Y_{k}^{c_{\lambda}}$ is a direct summand of $Y_{k}$, it also has a flag of generalized Verma modules. Now $\bar{\Omega}$ acts on $V(\overline{\mathfrak{g}}, \mu)\left(\mu \in \mathcal{P}_{\overline{\mathfrak{l}}}^{+}\right)$as the scalar $\left(\mu+2 \rho_{s} \mid \mu\right)_{s}$, and hence $Y_{k}^{c_{\lambda}}$ consists of those $V(\overline{\mathfrak{g}}, \mu)$ in $Y_{k}$ with $c_{\lambda}=\left(\mu+2 \rho_{s} \mid \mu\right)_{s}$. But such $\mu$ 's are precisely of the form $\theta\left(w \circ \Lambda^{\mathfrak{x}}(\lambda)\right)$ by Corollary 5.2 .

\section{7. $\mathfrak{u}_{-}$-HOMOLOGY FORMULA OF $\widehat{\mathfrak{g l}}_{\infty}$-MODULES AT NEGATIVE INTEGRAL LEVELS}

In this section we apply our method to obtain a Kostant-type homology formula for irreducible highest weight $\widehat{\mathfrak{g l}}_{\infty}$-modules that appear in bosonic Fock spaces. These representations have negative integral levels and have been studied in the context of vertex algebras in $[\mathrm{KR}]$.

We fix a positive integer $\ell \geq 1$. Consider $\ell$ pairs of free bosons $\gamma^{ \pm, i}(z)$ with $i=$ $1, \cdots, \ell$ (see Section 3.3). Let $\mathfrak{F}^{-\ell}$ denote the corresponding Fock space generated by the vaccum vector $|0\rangle$, which is annihilated by $\gamma_{r}^{ \pm, i}$ for $r>0$.

Given $\lambda \in \mathcal{P}(\mathrm{GL}(\ell))$ with $\lambda_{1} \geq \cdots \geq \lambda_{i}>0=\cdots=0>\lambda_{j+1} \geq \cdots \geq \lambda_{\ell}$, we define

$$
\Lambda_{-}^{\mathfrak{a}}(\lambda)=\sum_{k=1}^{i} \lambda_{k} \epsilon_{k}+\sum_{k=j+1}^{\ell} \lambda_{k} \epsilon_{k-\ell}-\ell \Lambda_{0}^{\mathfrak{a}} .
$$

There exists a joint action of $\left(\widehat{\mathfrak{g l}}_{\infty}, \mathrm{GL}(\ell)\right)$ on $\mathfrak{F}^{-\ell}$, giving rise to the following multiplicity-free decomposition:

Proposition 7.1. [KR] (cf. [W, Theorem 5.1]) As a $\left(\widehat{\mathfrak{g l}}_{\infty}, \mathrm{GL}(\ell)\right)$-module, we have

$$
\mathfrak{F}^{-\ell} \cong \bigoplus_{\lambda \in \mathcal{P}(\mathrm{GL}(\ell))} L\left(\widehat{\mathfrak{g l}}_{\infty}, \Lambda_{-}^{\mathfrak{a}}(\lambda)\right) \otimes V_{\mathrm{GL}(\ell)}^{\lambda}
$$


Computing the trace of the operator $\prod_{n \in \mathbb{Z}} x_{n}^{E_{n n}} \prod_{i=1}^{\ell} z_{i}^{e_{i i}}$ on both sides of (7.1) (see [W] for the action of $\left(\widehat{\mathfrak{g l}}_{\infty}, \mathrm{GL}(\ell)\right)$ on $\left.\mathfrak{F}^{-\ell}\right)$, we obtain the following identity:

$$
\frac{1}{\prod_{i=1}^{\ell} \prod_{n \in \mathbb{N}}\left(1-x_{n} z_{i}\right)\left(1-x_{1-n}^{-1} z_{i}^{-1}\right)}=\sum_{\lambda \in \mathcal{P}(\operatorname{GL}(\ell))} \operatorname{ch} L\left(\widehat{\mathfrak{g} l_{\infty}}, \Lambda_{-}^{\mathfrak{a}}(\lambda)\right) \operatorname{ch} V_{\mathrm{GL}(\ell)}^{\lambda} .
$$

Theorem 7.1. For $\lambda \in \mathcal{P}(\mathrm{GL}(\ell))$, we have

$$
\operatorname{ch} L\left(\widehat{\mathfrak{g l}}_{\infty}, \Lambda_{-}^{\mathfrak{a}}(\lambda)\right)=\frac{\sum_{k=0}^{\infty} \sum_{w \in W_{k}^{0}}(-1)^{k} s_{\left(\lambda_{w}^{+}\right)^{\prime}}\left(x_{1}, x_{2}, \cdots\right) s_{\left(\lambda_{w}^{-}\right)^{\prime}}\left(x_{0}^{-1}, x_{-1}^{-1}, \cdots\right)}{\prod_{i, j \in \mathbb{N}}\left(1-x_{-i+1}^{-1} x_{j}\right)} .
$$

Proof. The proof is similar to that of Theorem 3.3. Using (2.17) and (2.18) we may view (2.5) as a symmetric function identity in the variables $\left\{x_{i}^{-1}\right\}_{i \in-\mathbb{Z}_{+}}$and $\left\{x_{i}\right\}_{i \in \mathbb{N}}$. We then apply to this new identity the involution of the ring of symmetric functions that interchanges the elementary symmetric functions with the complete symmetric functions. Comparing with (7.2), we obtain the required formula.

Let $\mathfrak{u}_{ \pm}$and $\mathfrak{l}$ be as in Section 2.4. For $\lambda \in \mathcal{P}(\mathrm{GL}(\ell))$, define the homology groups $\mathrm{H}_{k}\left(\mathfrak{u}_{-} ; L\left(\widehat{\mathfrak{g l}}_{\infty}, \Lambda_{-}^{\mathfrak{a}}(\lambda)\right)\right)$ in the same way. One can check that $\mathrm{H}_{k}\left(\mathfrak{u}_{-} ; L\left(\widehat{\mathfrak{g l}}_{\infty}, \Lambda_{-}^{\mathfrak{a}}(\lambda)\right)\right)$ is completely reducible as an $\mathfrak{l}$-module, and if $L(\mathfrak{l}, \eta)$ is a irreducible component, then $\left(\eta+2 \rho_{c} \mid \eta\right)_{c}=\left(\Lambda_{-}^{\mathfrak{a}}(\lambda)+2 \rho_{c} \mid \Lambda_{-}^{\mathfrak{a}}(\lambda)\right)_{c}(\mathrm{cf}$. [J, L] $)$.

Suppose that $\mu=\sum_{i \in \mathbb{N}} \mu_{i} \epsilon_{i}-\sum_{j \in-\mathbb{Z}_{+}} \mu_{j} \epsilon_{j}+c \Lambda_{0}^{\mathfrak{a}} \in \mathcal{P}_{\mathfrak{l}}^{+}$is given. Put $\left(\mu_{1}, \mu_{2}, \ldots\right)^{\prime}=$ $\left(\mu_{1}^{\prime}, \mu_{2}^{\prime}, \ldots\right)$ and $\left(\mu_{0}, \mu_{-1}, \ldots\right)^{\prime}=\left(\mu_{0}^{\prime}, \mu_{-1}^{\prime}, \ldots\right)$, and define

$$
\vartheta(\mu)=\sum_{i \in \mathbb{N}} \mu_{i}^{\prime} \epsilon_{i}-\sum_{j \in-\mathbb{Z}_{+}} \mu_{j}^{\prime} \epsilon_{j}-c \Lambda_{0}^{\mathfrak{a}}
$$

Note that $\vartheta(\mu) \in \mathcal{P}_{\mathfrak{l}}^{+}$and $\vartheta^{2}$ is the identity on $\mathcal{P}_{\mathfrak{l}}^{+}$. In particular, we have $\vartheta\left(\Lambda^{\mathfrak{a}}(\lambda)\right)=$ $\Lambda_{-}^{\mathfrak{a}}(\lambda)$.

Let $\omega$ be the map which has been used in the proof of Theorem 17.1. That is, $\omega\left(\operatorname{ch} L\left(\widehat{\mathfrak{g l}}, \Lambda^{\mathfrak{a}}(\lambda)\right)\right)=\operatorname{ch} L\left(\widehat{\mathfrak{g l}}, \Lambda_{-}^{\mathfrak{a}}(\lambda)\right), \omega(\operatorname{ch} L(\mathfrak{l}, \mu))=\operatorname{ch} L(\mathfrak{l}, \vartheta(\mu))$ for $\mu \in \mathcal{P}_{\mathfrak{l}}^{+}$, and

$$
\omega\left(\operatorname{ch}\left[\Lambda^{k} \mathfrak{u}_{-} \otimes L\left(\widehat{\mathfrak{g l}}_{\infty}, \Lambda^{\mathfrak{a}}(\lambda)\right)\right]\right)=\operatorname{ch}\left[\Lambda^{k} \mathfrak{u}_{-} \otimes L\left(\widehat{\mathfrak{g l}}_{\infty}, \Lambda_{-}^{\mathfrak{a}}(\lambda)\right)\right]
$$

Using similar arguments as in Lemma 5.2, we can check that for $\mu \in \mathcal{P}_{\mathfrak{l}}^{+}, L(\mathfrak{l}, \mu)$ is a component in $\Lambda^{k} \mathfrak{u}_{-} \otimes L\left(\widehat{\mathfrak{g l}}_{\infty}, \Lambda^{\mathfrak{a}}(\lambda)\right)$ if and only if $L(\mathfrak{l}, \vartheta(\mu))$ is a component in $\Lambda^{k} \mathfrak{u}_{-} \otimes L\left(\widehat{\mathfrak{g l}}_{\infty}, \Lambda_{-}^{\mathfrak{a}}(\lambda)\right)$. Furthermore, they have the same multiplicity.

To compare the eigenvalues of the Casimir operator $\Omega$ on the irreducible components in homology groups, we need the following lemma.

Lemma 7.1. CZ1, Lemma 4.7] For $\lambda \in \mathcal{P}^{+}$, we have

$$
\begin{aligned}
& \left(\lambda+2 \rho_{1} \mid \lambda\right)_{1}=-\left(\lambda^{\prime}+2 \rho_{1} \mid \lambda^{\prime}\right)_{1}-2|\lambda|, \\
& \left(\lambda+2 \rho_{2} \mid \lambda\right)_{2}=-\left(\lambda^{\prime}+2 \rho_{2} \mid \lambda^{\prime}\right)_{2}+2|\lambda| .
\end{aligned}
$$


Lemma 7.2. Let $\lambda \in \mathcal{P}(\mathrm{GL}(\ell))$ and $\mu \in \mathcal{P}_{\mathfrak{l}}^{+}$such that $L(\mathfrak{l}, \mu)$ is a component in $\Lambda^{k} \mathfrak{u}_{-} \otimes L\left(\widehat{\mathfrak{g l}}_{\infty}, \Lambda^{\mathfrak{a}}(\lambda)\right)$. Then $\left(\Lambda^{\mathfrak{a}}(\lambda)+2 \rho_{c} \mid \Lambda^{\mathfrak{a}}(\lambda)\right)_{c}=\left(\mu+2 \rho_{c} \mid \mu\right)_{c}$ if and only if $\left(\Lambda_{-}^{\mathfrak{a}}(\lambda)+2 \rho_{c} \mid \Lambda_{-}^{\mathfrak{a}}(\lambda)\right)_{c}=\left(\vartheta(\mu)+2 \rho_{c} \mid \vartheta(\mu)\right)_{c}$.

Proof. Suppose that $\mu=\sum_{i \in \mathbb{N}} \mu_{i} \epsilon_{i}-\sum_{j \in-\mathbb{Z}_{+}} \mu_{j} \epsilon_{j}+\ell \Lambda_{0}^{\mathfrak{a}}$. Put $\mu^{+}=\left(\mu_{1}, \mu_{2}, \ldots\right)$ and $\mu^{-}=\left(\mu_{0}, \mu_{-1}, \ldots\right)$. Then

$$
\begin{aligned}
& \left(\mu+2 \rho_{c} \mid \mu\right)_{c} \\
& =\sum_{i \in \mathbb{N}} \mu_{i}\left(\mu_{i}-2 i\right)+\sum_{i \in \mathbb{N}} \mu_{1-i}\left(\mu_{1-i}-2 i\right)-\ell\left(\sum_{i \in \mathbb{N}} \mu_{i}+\sum_{i \in-\mathbb{Z}_{+}} \mu_{i}\right) \\
& =\left(\mu^{+}+2 \rho_{1} \mid \mu^{+}\right)_{1}+\left(\mu^{-}+2 \rho_{2} \mid \mu^{-}\right)_{2}-\ell\left(\sum_{i \in \mathbb{N}} \mu_{i}+\sum_{i \in-\mathbb{Z}_{+}} \mu_{i}\right) .
\end{aligned}
$$

On the other hand, put $\left(\mu^{+}\right)^{\prime}=\left(\mu_{1}^{\prime}, \mu_{2}^{\prime}, \ldots\right)$ and $\left(\mu^{-}\right)^{\prime}=\left(\mu_{0}^{\prime}, \mu_{-1}^{\prime}, \ldots\right)$. Then

$$
\begin{aligned}
& \left(\vartheta(\mu)+2 \rho_{c} \mid \vartheta(\mu)\right)_{c} \\
& =\sum_{i \in \mathbb{N}} \mu_{i}^{\prime}\left(\mu_{i}^{\prime}-2 i\right)+\sum_{i \in \mathbb{N}} \mu_{1-i}^{\prime}\left(\mu_{1-i}^{\prime}-2 i\right)+\ell\left(\sum_{i \in \mathbb{N}} \mu_{i}^{\prime}+\sum_{i \in-\mathbb{Z}_{+}} \mu_{i}^{\prime}\right) \\
& =\left(\left(\mu^{+}\right)^{\prime}+2 \rho_{1} \mid\left(\mu^{+}\right)^{\prime}\right)_{1}+\left(\left(\mu^{-}\right)^{\prime}+2 \rho_{2} \mid\left(\mu^{-}\right)^{\prime}\right)_{2}+\ell\left(\sum_{i \in \mathbb{N}} \mu_{i}^{\prime}+\sum_{i \in-\mathbb{Z}_{+}} \mu_{i}^{\prime}\right) \\
& =-\left(\mu^{+}+2 \rho_{1} \mid \mu^{+}\right)_{1}-2 \sum_{i \in \mathbb{N}} \mu_{i}-\left(\mu^{-}+2 \rho_{2} \mid \mu^{-}\right)_{2}+2 \sum_{i \in-\mathbb{Z}_{+}} \mu_{i}+\ell\left(\sum_{i \in \mathbb{N}} \mu_{i}+\sum_{i \in-\mathbb{Z}_{+}} \mu_{i}\right) \\
& =-\left(\mu+2 \rho_{c} \mid \mu\right)_{c}-2\left(\mu-\ell \Lambda_{0}^{\mathfrak{a}} \mid \sum_{i \in \mathbb{Z}} \epsilon_{i}\right)_{c} .
\end{aligned}
$$

Then from the above equations, we observe that

$$
\begin{aligned}
& \left(\Lambda^{\mathfrak{a}}(\lambda)+2 \rho_{c} \mid \Lambda^{\mathfrak{a}}(\lambda)\right)_{c}-\left(\mu+2 \rho_{c} \mid \mu\right)_{c} \\
& =-\left(\Lambda_{-}^{\mathfrak{a}}(\lambda)+2 \rho_{c} \mid \Lambda_{-}^{\mathfrak{a}}(\lambda)\right)_{c}+\left(\vartheta(\mu)+2 \rho_{c} \mid \vartheta(\mu)\right)_{c}-2\left(\Lambda^{\mathfrak{a}}(\lambda)-\mu \mid \sum_{i \in \mathbb{Z}} \epsilon_{i}\right)_{c} .
\end{aligned}
$$

Since $\Lambda^{\mathfrak{a}}(\lambda)-\mu$ is a sum of positive roots, we have $\left(\Lambda^{\mathfrak{a}}(\lambda)-\mu \mid \sum_{i \in \mathbb{Z}} \epsilon_{i}\right)_{c}=0$. This completes the proof.

Using the same arguments as in Theorem 5.1 together with Lemma 7.2 , we conclude the following.

Theorem 7.2. Let $\lambda \in \mathcal{P}(\mathrm{GL}(\ell)), k \in \mathbb{Z}_{+}$, and $\vartheta$ as in (7.3). Then as $\mathfrak{l}$-modules, we have

$$
\mathrm{H}_{k}\left(\mathfrak{u}_{-} ; L\left(\widehat{\mathfrak{g l}}_{\infty}, \Lambda_{-}^{\mathfrak{a}}(\lambda)\right) \cong \bigoplus_{w \in W_{k}^{0}} L\left(\mathfrak{l}, \vartheta\left(w \circ \Lambda^{\mathfrak{a}}(\lambda)\right)\right) .\right.
$$

In particular, we have $\operatorname{ch}\left[\mathrm{H}_{k}\left(\mathfrak{u}_{-} ; L\left(\widehat{\mathfrak{g l}}_{\infty}, \Lambda_{-}^{\mathfrak{a}}(\lambda)\right)\right]=\omega\left(\operatorname{ch}\left[\mathrm{H}_{k}\left(\mathfrak{u}_{-} ; L\left(\widehat{\mathfrak{g l}}_{\infty}, \Lambda^{\mathfrak{a}}(\lambda)\right)\right]\right)\right.\right.$. 
For $\nu \in \mathcal{P}_{\mathfrak{l}}^{+}$, let $V(\nu)=U\left(\widehat{\mathfrak{g l}}_{\infty}\right) \otimes_{U(\mathfrak{p})} L(\mathfrak{l}, \nu)$ be the generalized Verma module, where $\mathfrak{p}=\mathfrak{l}+\mathfrak{u}_{+}$and $\mathfrak{u}_{+}$acts trivially on $L(\mathfrak{l}, \nu)$.

Corollary 7.1. For $\lambda \in \mathcal{P}(\mathrm{GL}(\ell))$, there exists a resolution of $\widehat{\mathfrak{g l}}_{\infty}$-modules

$$
\cdots \stackrel{d_{k+1}}{\longrightarrow} Z_{k} \stackrel{d_{k}}{\longrightarrow} Z_{k-1} \stackrel{d_{k-1}}{\longrightarrow} \cdots \stackrel{d_{1}}{\longrightarrow} Z_{0} \stackrel{d_{0}}{\longrightarrow} L\left(\widehat{\mathfrak{g l}}_{\infty}, \Lambda_{-}^{\mathfrak{a}}(\lambda)\right) \rightarrow 0
$$

such that $Z_{k}$ has a flag of generalized Verma modules, and as an $\mathfrak{l}+\mathfrak{u}_{-}$-module it is isomorphic to $\bigoplus_{w \in W_{k}^{0}} V\left(\vartheta\left(w \circ \Lambda^{\mathfrak{a}}(\lambda)\right)\right)$.

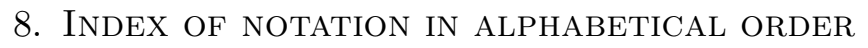

- $\mathfrak{F}^{\ell}$ : Fock space generated by $\ell$ pairs of free fermions defined in Section 2.3 ,

$\cdot \mathfrak{F}^{-\ell}$ : Fock space generated by $\ell$ pairs of free bosons defined in Section 7 ,

. $\overline{\mathfrak{F}} \ell, \overline{\mathfrak{F}}_{0}^{\ell}$ : Fock space generated by $\ell$ pairs of free fermions and $\ell$ pairs of free bosons defined in Section 3.3 ,

$\cdot \overline{\mathfrak{F}}^{\frac{1}{2}}$ : Fock space generated by a pair of neutral free fermion and free boson defined in Section 3.3 ,

$\cdot \mathfrak{g}$ : the Lie algebra $\widehat{\mathfrak{g l}}_{\infty}, \mathfrak{b}_{\infty}, \mathfrak{c}_{\infty}$ or $\mathfrak{d}_{\infty}$ of type $\mathfrak{x} \in\{\mathfrak{a}, \mathfrak{b}, \mathfrak{c}, \mathfrak{d}\}$, respectively,

$\cdot \overline{\mathfrak{g}}$ : the Lie superalgebra $\widehat{\mathfrak{g l}}_{\infty \mid \infty}, \widehat{\mathcal{B}}, \widehat{\mathfrak{C}}$ or $\widehat{\mathcal{D}}$ of type $\mathfrak{x} \in\{\mathfrak{a}, \mathfrak{b}, \mathfrak{c}, \mathfrak{d}\}$, respectively,

- $G$ : the Lie group $\mathrm{GL}(\ell), \operatorname{Pin}(2 \ell), \operatorname{Sp}(2 \ell)$ or $\mathrm{O}(m)$ defined in Section 2.3 ,

$\cdot \mathfrak{h}$ : a Cartan subalgebra of $\mathfrak{g}$

$\cdot \overline{\mathfrak{h}}$ : a Cartan subalgebra of $\overline{\mathfrak{g}}$,

- $H S_{\lambda}$ : Hook Schur function associated with $\lambda \in \mathcal{P}_{m \mid n}^{+}$, see Section 3.2.2,

- $I$ : an index set for simple roots for $\mathfrak{g}$ with $0 \in I$,

$\cdot \bar{I}$ : an index set for simple roots for $\overline{\mathfrak{g}}$ with $0 \in \bar{I}$,

$\cdot \mathfrak{l}$ : the subalgebra of $\mathfrak{g}$ with simple roots indexed by $I \backslash\{0\}$ (2.15),

$\cdot \overline{\mathfrak{l}}$ : the subalgebra of $\overline{\mathfrak{g}}$ with simple roots indexed by $\bar{I} \backslash\{0\}$ (4.6),

- $L(\mathfrak{g}, \Lambda)$ : the irreducible highest weight $\mathfrak{g}$-module of highest weight $\Lambda \in \mathfrak{h}^{*}$,

- $L(\overline{\mathfrak{g}}, \Lambda)$ : the irreducible highest weight $\overline{\mathfrak{g}}$-module of highest weight $\Lambda \in \overline{\mathfrak{h}}^{*}$,

- $L(\mathfrak{l}, \mu)$ : the irreducible $\mathfrak{l}$-module of highest weight $\mu \in \mathfrak{h}^{*}$,

- $L(\overline{\mathfrak{l}}, \mu)$ : the irreducible $\overline{\mathfrak{l}}$-module of highest weight $\mu \in \overline{\mathfrak{h}}^{*}$,

$\cdot \mathrm{L}\left(\mathfrak{g}, \Lambda^{\mathfrak{x}}(\lambda)\right), \mathrm{L}\left(\overline{\mathfrak{g}}, \bar{\Lambda}^{\mathfrak{x}}(\lambda)\right)$ : defined in (5.2),

$\cdot \mathrm{L}\left(\mathfrak{l}, w \circ \Lambda^{\mathfrak{x}}(\lambda)\right), \mathrm{L}\left(\overline{\mathfrak{l}}, \theta\left(w \circ \Lambda^{\mathfrak{x}}(\lambda)\right)\right):$ defined in (5.3)

$\cdot \Lambda^{\mathfrak{x}}(\lambda)$ : the highest weight of $\mathfrak{g}$-modules in Propositions 2.1 2.4 $\lambda \in \mathcal{P}(G)$,

- $\bar{\Lambda}^{\mathfrak{x}}(\lambda)$ : the highest weight of $\overline{\mathfrak{g}}$-modules in Propositions 3.1 3.4 $\lambda \in \mathcal{P}(G)$,

$\cdot \lambda_{w}, \lambda_{w}^{+}, \lambda_{w}^{-}$: weights in $\mathfrak{h}^{*}$ associated with $\lambda \in \mathcal{P}(G)$ and $w \in W$ defined in Section 2.4,

- $\mathcal{O}_{m \mid n}^{+}, \mathcal{O}_{m \mid n}^{++}$: categories of $\mathfrak{g l}(m \mid n)$-modules defined in Section 3.2.2,

- $\mathcal{O}_{\infty \mid \infty}^{++}$: category of $\mathfrak{g l}(\infty \mid \infty)$-modules defined in Section 3.2.3,

$\cdot \Omega$ : the Casimir operator of $\mathfrak{g}$, see Section 4.1 , 
$\cdot \bar{\Omega}$ : the Casimir operator of $\overline{\mathfrak{g}}$, see Section 4.2

- $\omega^{\mathfrak{r}}$ : a composition of involutions on the ring of symmetric functions such that $\operatorname{chL}\left(\overline{\mathfrak{g}}, \bar{\Lambda}^{\mathfrak{x}}(\lambda)\right)=\omega^{\mathfrak{x}}\left(\operatorname{chL}\left(\mathfrak{g}, \Lambda^{\mathfrak{x}}(\lambda)\right)\right)$, see Section 3.3.4,

- $\mathcal{P}(G)$ : the set of highest weights for irreducible $G$-modules in Propositions 2.1, 2.4,

$\cdot \mathcal{P}^{+}$: the set of partitions,

- $\mathcal{P}_{\mathfrak{l}}^{+}$: the set of $\mathfrak{l}$-dominant weights,

- $\mathcal{P}_{\overline{\mathrm{l}}}^{+}$: a set of weights given in Section 4.3,

- $\mathcal{P}_{m \mid n}^{+}:(m \mid n)$-hook partitions defined in Section 3.2.2.

$\cdot \overline{\mathfrak{p}}:=\overline{\mathfrak{l}} \oplus \overline{\mathfrak{u}}_{+}$(4.6),

- $\rho_{c}$ : "half sum" of the positive roots of $\mathfrak{g}$, see Sections 2.2 .1 and 2.2 .2 ,

- $\rho_{s}$ : "half sum" of the positive roots of $\overline{\mathfrak{g}}$, see Section 4.2 ,

- $\rho_{1}, \rho_{2}$ : defined in Section 5.1 ,

- $s_{\lambda}$ : Schur function associated with $\lambda \in \mathcal{P}^{+}$,

- $\theta$ : a bijection from $\mathcal{P}_{\mathfrak{l}}^{+}$to $\mathcal{P}_{\overline{\mathfrak{l}}}^{+}$with $\theta\left(\Lambda^{\mathfrak{x}}(\lambda)\right)=\bar{\Lambda}^{\mathfrak{x}}(\lambda)$, see (5.1),

- $\theta_{1}, \theta_{2}$ : defined in Section 5.1 ,

$\cdot \mathfrak{u}_{ \pm}$: the nilradicals of $\mathfrak{g}$ with $\mathfrak{g}=\mathfrak{u}_{+} \oplus \mathfrak{l} \oplus \mathfrak{u}_{-}$(2.15),

$\cdot \overline{\mathfrak{u}}_{ \pm}$: the nilradicals of $\overline{\mathfrak{g}}$ with $\overline{\mathfrak{g}}=\overline{\mathfrak{u}}_{+} \oplus \overline{\mathfrak{l}} \oplus \overline{\mathfrak{u}}_{-}$(4.6)

$\cdot V(\nu):=U(\overline{\mathfrak{g}}) \otimes_{U(\overline{\mathfrak{p}})} L(\overline{\mathfrak{l}}, \nu)$ for $\nu \in \mathcal{P}_{\overline{\mathfrak{l}}}^{+}$,

- $W\left(\right.$ resp. $\left.W_{0}\right)$ : Weyl group of $\mathfrak{g}$ (resp. $\left.\mathfrak{l}\right)$,

- $W_{k}^{0}$ : the set of the minimal length representatives of $W_{0} \backslash W$ of length $k$,

- $w \circ \mu:=w\left(\mu+\rho_{c}\right)-\rho_{c}$ for $w \in W$ and $\mu \in \mathfrak{h}^{*}$,

- $\left(Y_{k}, d_{k}\right)$ : a standard resolution of $\mathrm{L}\left(\overline{\mathfrak{g}}, \bar{\Lambda}^{\mathfrak{x}}(\lambda)\right)$, see (6.1),

- $\left(Y_{k}^{c_{\lambda}}, d_{k}\right)$ : restriction of $\left(Y_{k}, d_{k}\right)$ to generalized $c_{\lambda}$-eigenspace of $\bar{\Omega}$, see (6.2),

- $\zeta$ : function on half-integers defined in Section 4.1,

$\cdot(\cdot \mid \cdot)_{c}$ : a symmetric bilinear form on $\mathfrak{h}^{*}$, see Section 4.1,

$\cdot(\cdot \mid \cdot)_{s}$ : a symmetric bilinear form on $\overline{\mathfrak{h}}^{*}$, see Section 4.2,

$\cdot(\cdot \mid \cdot)_{1},(\cdot \mid \cdot)_{2}$ : defined in Section 5.1 .

$\cdot\langle\cdot\rangle$ : function on integers defined in Section 3.2 .3 ,

\section{REFERENCES}

[A] F. Aribaud: Une nouvelle démonstration d'un théorème de R. Bott et B. Kostant, Bull. Soc. Math. France 95 (1967), 205-242.

[B] J. Brundan: Kazhdan-Lusztig polynomials and character formulae for the Lie superalgebra $\mathfrak{g l}(m \mid n)$, J. Amer. Math. Soc. 16 (2003), 185-231.

[BR] A. Berele and A. Regev: Hook Young diagrams with applications to combinatorics and representations of Lie superalgebras, Adv. Math. 64 (1987), 118-175. 
[BT] T. Bröcker and T. tom Dieck: Representations of compact Lie Groups, Springer Verlag, New York 1995.

[CL] S.-J. Cheng and N. Lam: Infinite-dimensional Lie superalgebras and hook Schur functions, Commun. Math. Phys. 238 (2003), 95-118.

[CW1] S.-J. Cheng and W. Wang: Howe duality for Lie superalgebras, Compositio Math. 128 (2001), 55-94.

[CW2] S.-J. Cheng and W. Wang: Lie subalgebras of differential operators on the super circle, Publ. Res. Inst. Math. Sci. 39 (2003), 545-600.

[CZ1] S.-J. Cheng and R. B. Zhang: Analogue of Kostant's u-cohomology formula for the general linear superalgebra, Int. Math. Res. Not. 2004 (2004), 31-53.

[CZ2] S.-J. Cheng and R. B. Zhang: Howe duality and combinatorial character formula for orthosymplectic Lie superalgebras, Adv. Math. 182 (2004), 124-172.

[CWZ] S.-J. Cheng, W. Wang and R. B. Zhang: Super duality and Kazhdan-Lusztig polynomials, Trans. Amer. Math. Soc., to appear, math.RT/0409016.

[DJKM] E. Date, M. Jimbo, M. Kashiwara and T. Miwa: Transformation groups for soliton equations. III. Operator approach to the Kadomtsev-Petviashvili equation, J. Phys. Soc. Japan 50 (1981), 3806-3812. Transformation groups for soliton equations. IV. A new hierarchy of soliton equations of KP-type, Phys. D 4 (1981/82), 343-365.

[F] I. Frenkel: Representations of affine Lie algebras, Hecke modular forms and Kortweg-de Vries type equations, Lect. Notes Math. 933 (1982), 71-110.

[Fu] D. B. Fuks: Cohomology of infinite-dimensional Lie algebras, Contemporary Soviet Mathematics, Consultants Bureau, New York, 1986.

[GL] H. Garland and J. Lepowsky: Lie algebra homology and the Macdonald-Kac formulas, Invent. Math. 34 (1976), 37-76.

[H1] R. Howe: Remarks on classical invariant theory, Trans. Amer. Math. Soc. 313 (1989), 539-570.

[H2] R. Howe: Perspectives on Invariant Theory: Schur Duality, Multiplicity-free Actions and Beyond, The Schur Lectures, Israel Math. Conf. Proc. 8, Tel Aviv (1992), 1-182.

[J] E. Jurisich: An exposition of generalized Kac-Moody algebras. Lie algebras and their representations (Seoul, 1995), 121-159, Contemp. Math. 194, Amer. Math. Soc., Providence, RI, 1996.

[K1] V. Kac, Lie superalgebras, Adv. Math. 16 (1977), 8-96.

[K2] V. Kac: Infinite-dimensional Lie algebras, Third edition, Cambridge University Press, Cambridge, 1990.

[KvL] V. Kac and J. van de Leur: Super boson-fermion correspondence, Ann. Inst. Fourier 37 (1987), 99-137.

[Ko] B. Kostant: Lie Algebra cohomology and the generalized Borel-Weil theorem, Ann. Math. 74 (1961), 329-387.

[KK] S. J. Kang and J. H. Kwon: Graded Lie superalgebras, supertrace formula, and orbit Lie superalgrbra, Proc. London Math. Soc. 81 (2000), 675-724.

[KR] V. Kac and A. Radul: Representation theory of the vertex algebra $W_{1+\infty}$, Transform. Groups 1 (1996), 41-70.

[KW] V. Kac and M. Wakimoto: Integrable highest weight modules over affine superalgebras and Appell's function, Commun. Math. Phys. 215 (2001), 631-682.

[L] L. Liu: Kostant's Formula for Kac-Moody Lie algebras, J. Algebra 149 (1992), 155-178. 
[LZ] N. Lam and R. B. Zhang: Quasi-finite modules for Lie superalgebras of infinite rank, Trans. Amer. Math. Soc. 358 (2006), 403-439.

[LS] D. Leites and I. Shchepochkina: The Howe duality and Lie superalgebras. Noncommutative structures in mathematics and physics (Kiev, 2000), 93-111, NATO Sci. Ser. II Math. Phys. Chem., 22, Kluwer Acad. Publ., Dordrecht, 2001.

[M] I. G. Macdonald: Symmetric functions and Hall polynomials, Oxford Math. Monogr., Clarendon Press, Oxford, 1995.

[S1] A. Sergeev: The tensor algebra of the identity representation as a module over the Lie superalgebras $g l(n, m)$ and $Q(n)$, Math. USSR Sbornik 51 (1985), 419-427.

[S2] A. Sergeev: An analogue of classical invariant theory for Lie superalgebras I, Michigan Math. J. 49 (2001), 113-146. An analogue of classical invariant theory for Lie superalgebras II, Michigan Math. J. 49 (2001), 147-168.

[Se] V. Serganova: Kazhdan-Lusztig polynomials and character formula for the Lie superalgebra $\mathfrak{g l}(m \mid n)$, Selecta Math. (N.S.) 2 (1996), 607-651.

[W] W. Wang: Duality in infinite dimensional Fock representations, Commun. Contem. Math. 1 (1999), 155-199.

Institute of Mathematics, Academia Sinica, Taipei, Taiwan 11529

E-mail address: chengsj@math.sinica.edu.tw

Department of Mathematics, University of Seoul, 90, Cheonnong-Dong, DongdaemunGu, SeOul 130-743, Korea

E-mail address: jhkwon@uos.ac.kr 